\title{
Epithelial cell plasticity drives endoderm formation during gastrulation
}

\author{
Katharina Scheibner ${ }^{1,2,3,12}$, Silvia Schirge ${ }^{1,2,3,12}$, Ingo Burtscher ${ }^{1,2,3,12}$, Maren Büttner ${ }^{4}$, Michael Sterr ${ }^{1,2,3}$, \\ Dapeng Yang ${ }^{5}$, Anika Böttcher ${ }^{10}{ }^{1,2,3}$, Ansarullah ${ }^{1,2,3}$, Martin Irmler ${ }^{6}{ }^{6}$, Johannes Beckers ${ }^{3,6,7}$, \\ Filippo M. Cernilogar $\mathbb{D}^{8}{ }^{\text {, Gunnar Schotta }}{ }^{8}$, Fabian J. Theis $\mathbb{C}^{4,9,10}$ and Heiko Lickert $\mathbb{C}^{1,2,3,11 凶}$
}

\begin{abstract}
It is generally accepted that epiblast cells ingress into the primitive streak by epithelial-to-mesenchymal transition (EMT) to give rise to the mesoderm; however, it is less clear how the endoderm acquires an epithelial fate. Here, we used embryonic stem cell and mouse embryo knock-in reporter systems to combine time-resolved lineage labelling with high-resolution single-cell transcriptomics. This allowed us to resolve the morphogenetic programs that segregate the mesoderm from the endoderm germ layer. Strikingly, while the mesoderm is formed by classical EMT, the endoderm is formed independent of the key EMT transcription factor Snail1 by mechanisms of epithelial cell plasticity. Importantly, forkhead box transcription factor A2 (Foxa2) acts as an epithelial gatekeeper and EMT suppressor to shield the endoderm from undergoing a mesenchymal transition. Altogether, these results not only establish the morphogenetic details of germ layer formation, but also have broader implications for stem cell differentiation and cancer metastasis.
\end{abstract}

T he recent single-cell genomics revolution has generated transcriptional and epigenetic roadmaps for the formation of the three principal germ layers during gastrulation ${ }^{1-3}$ Epithelial-to-mesenchymal transition (EMT) and mesenchymalto-epithelial transition (MET) are evolutionarily conserved processes that occur during development and are essential for gastrulation and embryonic morphogenesis, but if they are dysregulated in adulthood they lead to cancer metastasis ${ }^{4-6}$. Although gastrulation serves as the mechanistic basis to understand EMT, cancer metastasis, stem cell differentiation and congenital disease, its understanding is incomplete in mammals. During gastrulation, epiblast cells exit pluripotency and allocate to one of the three germ layers-the ectoderm, mesoderm or definitive endoderm lineage-to generate the progenitors of major organs in the body ${ }^{7}$. The T-box transcription factor Brachyury (T) and forkhead box transcription factor A2 (Foxa2) are master regulators of mesoderm and endoderm formation, respectively ${ }^{8-11}$. Morphogen gradients along the anterior-posterior axis induce posterior epiblast cells to undergo EMT and ingress into the primitive streak region (the space between the epiblast and visceral endoderm epithelial layers ${ }^{7,12,13}$. Upon high Wnt $/ \beta$-catenin, transforming growth factor- $\beta$ (TGF- $\beta$ ) and fibroblast growth factor signalling, EMT transcription factors such as Mesp1 and 2 (refs. ${ }^{14,15}$ ), Twist1 (ref. ${ }^{16}$ ), Snaill and 2 (refs. ${ }^{16-18}$ ) and Zeb1 and 2 (refs. ${ }^{19,20}$ ) are induced. Following the activation of an EMT program, columnar-shaped epiblast cells lose their epithelial morphology by dynamic expression changes and remodelling of apical-basal polarity and cell-cell adhesion. Simultaneously, cells adopt a mesenchymal fate by downregulation of E-cadherin and by upregulation of $\mathrm{N}$-cadherin, vimentin and $\alpha$-smooth muscle actin, resulting in ingression and migration of mesodermal cells into the primitive streak-classical hallmarks of EMT ${ }^{17,18,21-23}$. It is generally assumed that definitive endoderm (DE) progenitors undergo a further MET to give rise to the epithelial endoderm layer, in an EMT-MET cycle ${ }^{12,13,24-28}$; however, this idea is based on Drosophila and zebrafish model systems and has never been formally proven in mammals ${ }^{13}$. Previously, we noticed that before primitive streak formation and initiation of gastrulation, proximal $\mathrm{T}^{+}$mesoderm and distal Foxa $2^{+}$endoderm progenitors are already segregated in the epiblast ${ }^{11}$. As Foxa2 regulates axial mesendoderm (AME) and definitive endoderm differentiation, cell polarity and epithelialization ${ }^{11}$, we hypothesized that DE progenitors leave the epiblast to form the mature definitive endoderm lineage by a process independent of a complete EMT-MET cycle.

\section{Results}

Definitive endoderm is formed in the absence of an EMT-MET cycle. To test this hypothesis and specifically study endoderm differentiation and morphogenesis in more detail, we performed time-resolved and simultaneous lineage labelling and tracking using Foxa2-Venus fusion (FVF) and Sox17-mCherry fusion (SCF) double knock-in reporter mouse embryonic stem cells (mESCs) and mouse embryos ${ }^{29,30}$. During gastrulation, FVF ${ }^{\text {low }}$ epiblast progenitors upregulate FVF reporter activity while they leave the epithelium and give rise to FVF ${ }^{\text {high }}$ transitory progenitors (Extended Data Fig. 1a and Supplementary Video 1). FVFhigh transitory progenitors migrate between the epiblast and visceral endoderm layers until they upregulate SCF and intercalate into the outside visceral endoderm and give rise to the $\mathrm{FVF}^{\text {high }} / \mathrm{SCF}^{+}$definitive endoderm

IInstitute of Diabetes and Regeneration Research, Helmholtz Diabetes Center, Helmholtz Zentrum München, Munich, Germany. ${ }^{2}$ Institute of Stem Cell Research, Helmholtz Zentrum München, Munich, Germany. ${ }^{3}$ German Center for Diabetes Research (DZD), Munich, Germany. ${ }^{4}$ Institute of Computational Biology, Helmholtz Zentrum München, Munich, Germany. ${ }^{5}$ Developmental Biology Program, Sloan Kettering Institute, New York, NY, USA. 6 Institute of Experimental Genetics, Helmholtz Zentrum München, Munich, Germany. ${ }^{7}$ School of Life Sciences Weihenstephan, Technische Universität München, Freising, Germany. ${ }^{8}$ Division of Molecular Biology, Biomedical Center, Faculty of Medicine, Ludwig Maximilian University, Munich, Germany. ${ }^{9}$ Department of Mathematics, Technische Universität München, Munich, Germany. ${ }^{10}$ School of Life Sciences Weihenstephan, Technische Universität München, Freising, Germany. " ${ }^{11}$ School of Medicine, Klinikum Rechts der Isar, Technische Universität München, Munich, Germany. ${ }^{12}$ These authors contributed equally:

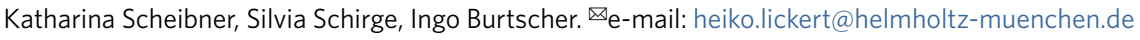


lineage $\mathrm{e}^{11,29,30}$. We recently noticed that it only takes $\sim 12 \mathrm{~h}$ for FVF/ SCF mESCs to differentiate from FVFlow progenitors into FVFigh/ $\mathrm{SCF}^{+}$definitive endoderm ${ }^{30}$. This short timespan seems insufficient for a cell to down- and upregulate molecular machineries on the messenger RNA (mRNA) and protein level to undergo a complete EMT-MET cycle. Thus, we investigated first whether Foxa $2^{\text {low }}$ epiblast progenitors leave the epithelium by EMT in early-, mid- and late-streak-stage embryos. Before gastrulation, FVF ${ }^{\text {low }}$ epiblast progenitors are found in the posterior epiblast region, suggesting that epiblast cells are already fate specified (Extended Data Fig. 1b) ${ }^{11}$. During primitive streak induction and elongation, FVF ${ }^{\text {low }}$ epiblast progenitors occupy an epiblast domain distal to the morphological anterior primitive streak (APS) region (Fig. 1a, $\mathrm{a}^{\prime}$ and Extended Data Fig. 1c). Within the FVF low epiblast domain, single FVF ${ }^{\text {high }}$ transitory progenitors appear, leave the epithelium and squeeze as elongated cells between the epiblast and visceral endoderm (Fig. 1a-c, insets $)^{11,17}$. This is in stark contrast with the proximal primitive streak, where $\mathrm{T}^{+}$mesoderm cells with a mesenchymal morphology span several cell diameters across the primitive streak region (Fig. 1a-c). During gastrulation, mesoderm formation is driven by the key EMT transcription factor Snaill (refs. ${ }^{17,18}$ ). At the mid- to late-streak stage, a distinct separation of three populations in the primitive streak region was apparent (see the schematic in Fig. $\left.1 b^{\prime}\right)$ : a large $\mathrm{T}^{+}$mesoderm population in the proximal primitive streak region; a few Foxa $2^{\text {high }} / \mathrm{T}^{+}$AME progenitors (Fig. 1b, $\mathrm{b}^{\prime}$ ); and Foxa $2^{\text {low }}$ epiblast progenitors in the epiblast and Foxa $2^{\text {high }}$ transitory progenitors that ingressed distally to the APS (Fig. 1a,b, $\mathrm{a}^{\prime}, \mathrm{b}^{\prime}$ and Extended Data Fig. 1a-d) ${ }^{11}$. Notably, the EMT transcription factor Snaill was highly upregulated in $\mathrm{T}^{+}$mesoderm progenitors, while it was weakly expressed on the mRNA level in Foxa $2^{\text {high }}$ transitory progenitors (Figs. 1b, $\mathrm{b}^{\prime}$ and Fig. 2e and Extended Data Fig. 1d) and not expressed in mature Foxa $2^{\text {high }} / \operatorname{Sox} 17^{+}$definitive endoderm (Extended Data Fig. 1e,g,h). During the transition to a mesenchymal state, Snaill downregulates E-cadherin ${ }^{17}$. At the same time, $\mathrm{N}$-cadherin is upregulated ${ }^{20}$. Immunostaining analysis revealed that mesodermal cells within the primitive streak displayed the well-described switch from E- to N-cadherin during EMT (Fig. 1c, $c^{\prime}$ and Extended Data Fig. 1f). In contrast, FVFigh transitory progenitors, definitive endoderm and AME cells maintained E-cadherin and synchronously upregulated $\mathrm{N}$-cadherin, as quantified by western blot and immunostaining (Fig. 1c-f and Extended Data Fig. 1f,i).

To understand the lineage bifurcations and hierarchy during gastrulation and to generate a continuous in vivo roadmap of the molecular changes, we combined FVF lineage labelling and flow sorting to perform high-throughput single-cell RNA sequencing (scRNA-seq) (Fig. 2a and Extended Data Fig. 2). This allowed us to enrich for rare transitory cell types and to map the $\mathrm{FVF}^{\text {low }}$ epiblast progenitors, FVF $^{\text {high }}$ transitory progenitors and FVF ${ }^{\text {high }}$ AME and definitive endoderm descendants via Louvain cluster annotation (Extended Data Fig. 2a-d). Using our previously established scVelo ${ }^{31}$ and CellRank ${ }^{32}$ algorithms, we combined directional information from the RNA velocity and robustness of trajectory inference to compute fate probabilities, and identified lineage driver genes during mesoderm and endoderm segregation (Fig. 2b-d and Extended Data Fig. 2e). Combining FVF lineage labelling with pseudotime analysis revealed that, during posterior epiblast-to-endoderm transition, EMT transcription factor genes (Snail1 and 2, Zeb1 and 2, Mesp1 and 2 and Twist1) are downregulated, whereas E-cadherin (Cdh1) is maintained and $\mathrm{N}$-cadherin (Cdh2) is upregulated (Fig. $2 \mathrm{~d}-\mathrm{f}$ ). In contrast, posterior epiblast cells undergoing a mesoderm transition upregulate an EMT transcription factor program and show an $E$ - to $N$-cadherin switch during mesenchymal transition. Altogether, these results suggest that, in mouse embryos, Foxa $2^{\text {low }}$ epiblast progenitors upregulate Foxa2 levels and give rise to Foxa $2^{\text {high }}$ transitory progenitors that ingress distal to the anatomical visible primitive streak to form the Foxa $2^{\text {high }} /$ Sox $17^{+}$definitive endoderm independent of a full EMT-MET cycle.

In vitro-generated definitive endoderm forms by partial EMT. To verify these results in vitro and simultaneously analyse mesoderm and endoderm segregation, we generated a $\mathrm{T}^{\mathrm{GFP} /+}$; Foxa $2^{\text {tagRFP/+ }}$ knock-in dual-reporter mESC line (Extended Data Fig. 3a). We differentiated the mESCs in a stepwise time-resolved manner into mesoderm and endoderm (Fig. $2 \mathrm{~g}$ and Methods) and sorted progenitors and definitive lineages by flow sorting using the reporter $\left(\mathrm{T}^{\mathrm{GFP}}\right.$ and Foxa2 $\left.{ }^{\text {tagRFP}}\right)$ and differential marker $\left(\mathrm{CD} 24^{\text {neg/low/high }}\right)$ expression (Extended Data Fig. 3b,c) ${ }^{33}$. Global transcriptional profiling at days 2 and 4 of differentiation revealed that mesoderm progenitors still express pluripotency markers (Pou5f1, Nanog and Sox2) and slightly upregulate mature mesoderm markers (Tbx3 and 5, Mesp1 and 2 and Hand1) (Fig. 2h). Similarly, DEPs still express pluripotency genes and already induce the expression of mature definitive endoderm markers (Sox17, Hhex, Dkk1 and Cerl) (Fig. 2h). Next, we tested whether EMT transcription factors are expressed during mesodermal and endodermal lineage acquisition in vitro. During mesoderm differentiation, EMT transcription factor genes (Zeb1 and 2, Mesp 1 and 2 and Snail1) are already expressed in mesoderm progenitors and further upregulated in mesoderm, while DEP and definitive endoderm do not upregulate EMT transcription factor genes (Fig. 2h). In vitro endoderm differentiations confirmed the absence of Snaill protein in DEPs and definitive endoderm (Extended Data Fig. 4a,b and Methods). Furthermore, E-cadherin and $N$-cadherin mRNA and protein were maintained or upregulated, respectively, during endoderm differentiation in vitro (Fig. $2 \mathrm{~h}$ and Extended Data Fig. 4c,d), consistent with our in vivo results (Fig. 1c-f). Using a previously generated knock-in Foxa2-H2B-Venus (Foxa2 $2^{\text {Venus } /+}$ ) mESC transcriptional reporter line ${ }^{34}$, we flow sorted Foxa2 $2^{\text {Venus low }}$ progenitor and Foxa $2^{\text {Venus high }}$ definitive endoderm, which maintain the epithelial marker protein E-cadherin during differentiation (Extended Data Fig. 4e-h). Re-analysis of our previously generated RNA-seq, chromatin immunoprecipitation sequencing (ChIP-seq) data from stem cell-derived endoderm ${ }^{34}$ suggested that the expression of $N$-cadherin as well as E-cadherin is regulated by Foxa2 (Fig. $2 \mathrm{~h}$ and Supplementary Table 1 ). Taken together, nascent endoderm expresses epithelial markers from specification to determination in vitro and in vivo. Furthermore, definitive endoderm is formed independent of an E- to N-cadherin switch and substantial upregulation of well-known EMT transcription factors, suggesting it is formed by partial EMT.

The EMT transcription factor Snaill is not required for endoderm formation. Several knockout studies have indicated redundant functions of the EMT transcription factors Zeb1 and 2 (refs. ${ }^{35,36}$ ) and Snail2 (ref. ${ }^{37}$ ) during gastrulation, whereas mutant embryos with alterations in Snaill fail to downregulate E-cadherin and show defective EMT and mesoderm formation ${ }^{17,18}$. To test whether Snaill function is required for definitive endoderm formation, we generated a Snail1 knockout mESC line (Extended Data Fig. 5a,b). After $3 \mathrm{~d}$ of definitive endoderm differentiation, we detected comparable numbers of definitive endoderm cells (Foxa $2^{\text {high }} /$ Sox $17^{+}$) formed from both wild-type (control) and Snail1 knockout mESCs (Fig. 3a-d and Extended Data Fig. 5c-f). To analyse definitive endoderm formation in vivo, we generated completely mESC-derived embryos by tetraploid aggregation (Fig. $3 \mathrm{e}-\mathrm{g})^{38}$. This allowed us to observe nascent definitive endoderm formation (derived from mESCs) and visceral endoderm dispersal (derived from tetraploid embryos expressing membrane Tomato $\left.(\mathrm{mT})^{39}\right)$ by means of fluorescent marker gene expression. At the early headfold stage, we observed that the visceral endoderm $\left(\mathrm{mT}^{+}\right)$was clearly dispersed by Snaill knockout mESC-derived definitive endoderm 
a

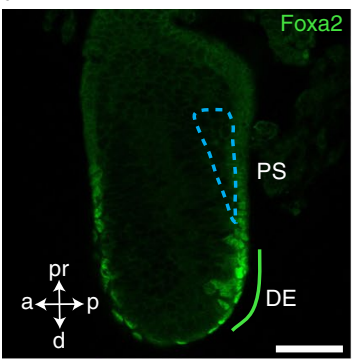

b
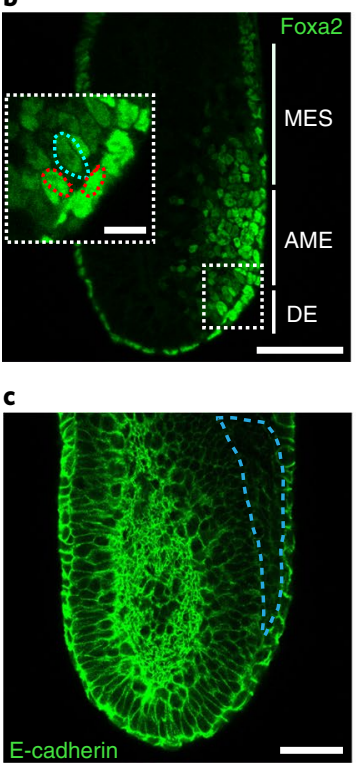

d

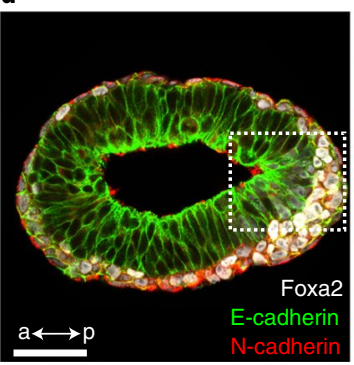

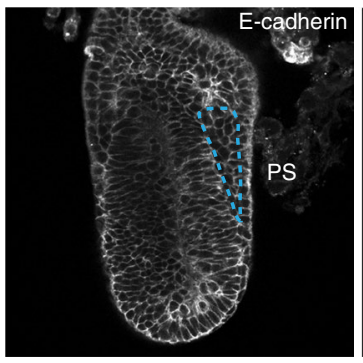
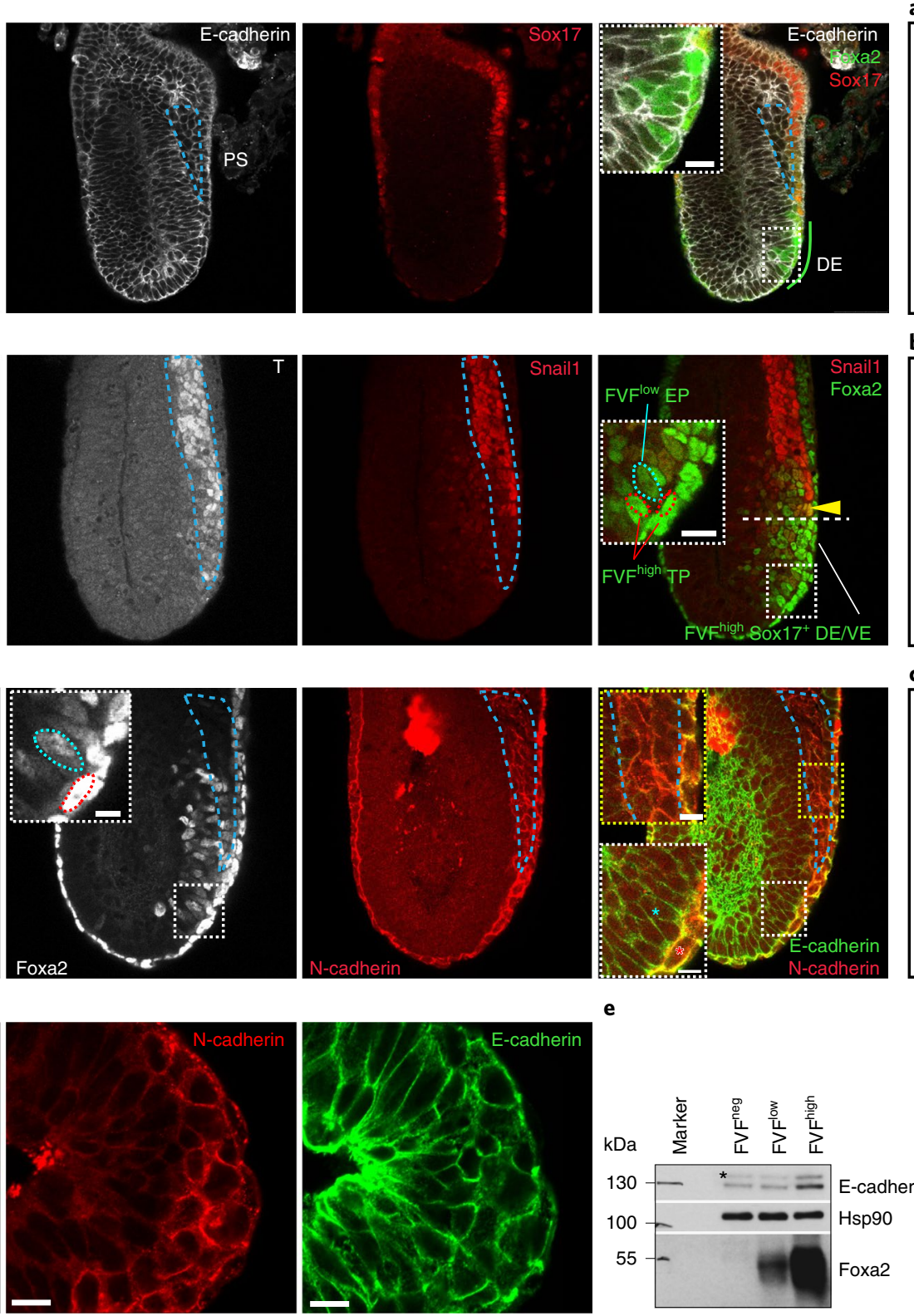
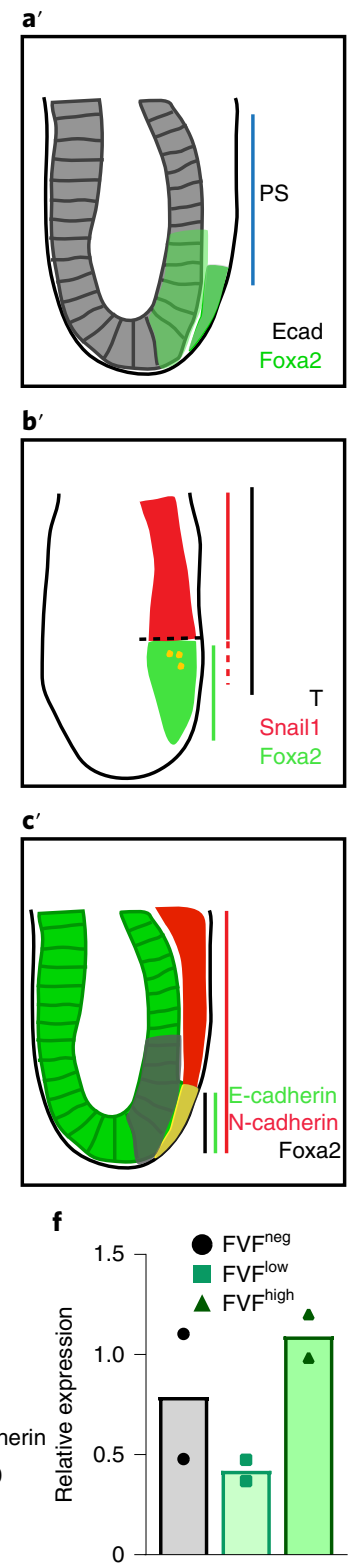

Fig. 1 | Endoderm progenitors do not show hallmarks of an EMT. a, Mid-streak-stage FVF/SCF embryos stained for Venus (Foxa2), E-cadherin and RFP (Sox17). The blue dashed line indicates the primitive streak (PS). a, anterior; d, distal; DE, definitive endoderm; p, posterior; pr, proximal. b. Immunohistochemistry of a mid-streak-stage FVF embryo stained for Venus (Foxa2), Snail1 and T. The blue dashed lines in the insets mark FVFlow

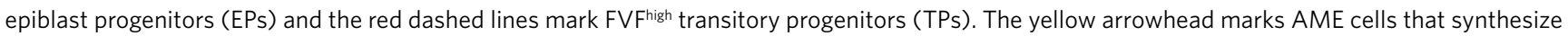
Foxa2, T and Snail1. The horizontal white dashed line indicates the border of Snail expression. MES, mesoderm; TP, transitory progenitor; VE, visceral endoderm. c, Mid-streak-stage FVF embryo immunostained for Venus (Foxa2; white), N-cadherin and E-cadherin. The blue asterisk (rightmost image inset in white dashed box) and inset blue dashed line (inset in image second from left) mark FVFlow epiblast progenitors in the epiblasts, whereas the red asterisk (rightmost image inset in white dashed box) and red dashed line (inset in image second from left) indicate FVFhigh transitory progenitors that express $\mathrm{E}$-cadherin and $\mathrm{N}$-cadherin. The primitive streak region is indicated by $\mathrm{N}$-cadherin expression (blue dashed line in rightmost image inset in dashed yellow box). $\mathbf{a}^{\prime}-\mathbf{c}^{\prime}$, Depiction of Foxa2 ( $\left.\mathbf{a}^{\prime}\right), \mathrm{T}$ and Snail $\left(\mathbf{b}^{\prime}\right)$ and E-cadherin and $\mathrm{N}$-cadherin expression ( $\mathbf{c}^{\prime}$ ) in gastrulating embryos based on the embryos in a-c. d, Transverse section through the epiblast of a mid-streak-stage wild-type embryo immunostained for Foxa2, E-cadherin and N-cadherin. e,f, Western blot analysis (e) and quantification (f) of E-cadherin from FACS-sorted FVFreg, FVFlow and FVFhigh cells of 122 and 36 FVF embryos ( $n=2$ ). The asterisk marks unspecific bands. All shown confocal images are single $z$ planes of a z stack. The images in a-d are representative of eight, three, three and three embryos, respectively. All samples were derived from biologically independent experiments. The data are presented as mean values. Scale bars, $50 \mu \mathrm{m}$ (insets, $10 \mu \mathrm{m}$ ).

$\left(\right.$ Sox $\left.17^{+}\right)$, comparable to control chimeras (Fig. 3e-g). Together, these findings demonstrate that the master EMT transcription factor Snail1 is not necessary for definitive endoderm formation in vitro and in vivo.
Foxa2 suppresses a complete EMT during endoderm formation. To better understand how definitive endoderm is formed during gastrulation, we investigated the role of Foxa2, which has been shown to be crucially important for the formation of epithelial lineages 
(that is, $\mathrm{ADE}$ and $\mathrm{AME})^{9,10}$. For this purpose, we used our previously established knock-in/knockout Foxa2 reporter line for endoderm differentiations and re-analysed our RNA-seq and ChIP-seq datasets $^{34}$. As predicted, we observed a lack of definitive endoderm formation and mature marker expression using the homozygous Foxa $2^{\text {Venus/Venus }}$ compared with heterozygous Foxa $2^{\text {Venus/+ }}$ definitive endoderm cells (Fig. 4a,b). This resembled the well-known phenotype of Foxa2 mutant embryos ${ }^{9-11,35}$. Interestingly, we noted that, upon loss of Foxa2, EMT transcription factor genes (Snail1, Mesp 1, Tbx3 and Zeb1 and 2) and the EMT marker gene (Vim) were upregulated in Foxa2 ${ }^{\text {Venus/Venus }}$ mutant cells (Fig. $4 \mathrm{~b}-\mathrm{d}$ ). To analyse whether Snail1 is also upregulated in the Foxa2 lineage in vivo, we generated mESC-derived embryos using heterozygous Foxa2 ${ }^{\text {Venus } /+}$ and homozygous Foxa2 ${ }^{\text {Venus/Venus }}$ knock-in/knockout mESCs by tetraploid complementation (Fig. $4 \mathrm{e}-\mathrm{h}$ ). At the late-streak stage, the APS region was thickened due to an accumulation of Foxa2 lineage-positive cells in the Foxa $2^{\text {Venus/Venus }} \mathrm{mESC}$-derived embryo (compare Fig. 4f,g) ${ }^{11}$. We also noticed more Venus lineage-labelled Foxa2 mutant cells with clear upregulation of the EMT transcription factor Snail1 (Fig. 4f-h). Induction of Snail1 expression in the Foxa $2^{\text {Venus/Venus }}$ mutant lineage-labelled cells in vitro as well as in vivo implied that Foxa2 suppresses the key EMT transcription factor Snaill to prevent E-cadherin downregulation and mesenchymal transition.

Foxa2 serves as an epithelial gatekeeper in the endoderm. TGF- $\beta$ and nodal and canonical Wnt $/ \beta$-catenin signalling activates EMT transcription factors and initiates gastrulation ${ }^{40-42}$. Recently, it was shown in different cancer and epithelial cell lines that the EMT key regulator Snaill is induced by the TGF- $\beta$ ligand 1 (ref. ${ }^{43}$ ) and Wnt $/ \beta$-catenin activation by Gsk $3 \beta$ inhibition ${ }^{37,44,45}$. In FVF ${ }^{\text {high }}$ transitory progenitors and definitive endoderm, we did not observe substantial upregulation of Snaill expression and synthesis (Figs. 1

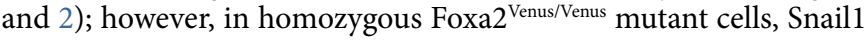
was highly expressed (Fig. $4 \mathrm{c}-\mathrm{h}$ ), suggesting that Foxa2 is a potential repressor of Snaill. However, ChIP-seq analysis of definitive endoderm cells revealed that Snail1 cis-regulatory elements are not directly bound by Foxa2 (Fig. $2 \mathrm{~h}$ and Supplementary Table 1$)^{34}$. Next, we analysed the activity of Wnt signalling genes and observed that canonical Wnt ligands and its targets, such as Wnt3 and APC, are not upregulated in definitive endoderm, in contrast with $\mathrm{T}^{+}$mesoderm, which highly expresses these genes (Fig. 5a,b). Interestingly, secreted inhibitors of the canonical Wnt $/ \beta$-catenin pathway (Cer1, Dkk1 and Srfp1 and 5) are highly upregulated in definitive endoderm but downregulated in Foxa2 ${ }^{\text {Venus/Venus }}$ knockout cells (Figs. $4 \mathrm{~b}$ and $5 \mathrm{a}, \mathrm{b}$ ). Further analysis of the ChIP-seq data suggested that several of the secreted inhibitors of the canonical Wnt/ $\beta$-catenin pathway are directly regulated by Foxa2, such as Cer1 (Fig. 5b and Supplementary Table 1). During gastrulation, we observed downregulation of Wnt ligands (Wnt3 and Wnt3a) and the target gene (Lef1), as well as upregulation of secreted Wnt inhibitors (Cer1, Dkk1 and Sfrp1 and 5) in the transition from the posterior epiblast to transitory progenitors and definitive endoderm (Fig. 5d,e). Interestingly, the AVE and ADE marker protein Cer1 is already upregulated in Foxa $2^{\text {high }}$ transitory progenitors and definitive endoderm at the posterior side of gastrula embryos (Figs. $5 \mathrm{f}$ and $6 a, b)$, which correlates with the downregulation of the Wnt target gene and transcription factor Lef1 (Fig. 6a-d). In line with our Foxa2 knockout results from in vitro differentiations (Fig. 4b), we also observed a lack of Cer1 expression in Venus ${ }^{+}$lineage-labelled cells of Foxa $2^{\text {Venus/Venus }}$ aggregation chimeras (Fig. $5 \mathrm{~g}$ ) and concomitant upregulation of the Wnt target gene Lef1 and the EMT transcription factor Snail1 (Fig. 6e-g). These results suggest that Foxa2 directly induces the expression of Wnt inhibitors and thereby indirectly inhibits Wnt/ $\beta$-catenin signalling and target genes, such as Lef1 and Snail1 (Fig. 6h) ${ }^{37,44-47}$.

To test whether inhibition of canonical Wnt signalling represses Snaill expression and promotes epithelial endoderm formation, we differentiated $\mathrm{T}^{\mathrm{GFP} /+}$; Foxa $2^{\mathrm{tagRFP} /+}$ mESCs for $1 \mathrm{~d}$ with addition of the Gsk3 $\beta$ inhibitor CHIR and activin A to induce endoderm formation (Extended Data Fig. 6a-d). Next, we supplemented the medium with the Wnt inhibitor Dickkopf 1 (DKK1) or the Wnt ligand secretion inhibitor IWP2 for the following $2 \mathrm{~d}$. The cells treated with Wnt inhibitors showed decreased Snaill expression compared with control cells, which was expected and confirmed that Snaill is a $\mathrm{Wnt} / \beta$-catenin target gene $\mathrm{e}^{37,44,45}$. Interestingly, the Foxa $2^{+} /$Sox $17^{+}$ definitive endoderm population was increased from $\sim 11$ to $\sim 51 \%$ in DKK1-supplemented cultures and $~ 60 \%$ in IWP2-supplemented cultures (Extended Data Fig. 6e-g). Altogether, these results show that Foxa 2 activates Wnt/ $\beta$-catenin inhibitors to indirectly repress Snail1 expression to promote progenitor differentiation towards an epithelial endodermal fate in vitro and in vivo.

During endoderm formation, Foxa2 regulates a molecular program to induce and maintain endodermal polarization and epithelialization ${ }^{11,48}$. Consistently, we observed that FVFigh transitory progenitors and $\mathrm{FVF}^{\text {high }} /$ Sox $17^{+}$definitive endoderm descendants do not undergo a complete EMT-MET cycle, but instead acquire epithelial cell plasticity to change their morphology from a columnarto a squamous-shaped epithelium (Figs. 1 and 2, Extended Data Fig. 1a and Supplementary Video 1$)^{11,29,30}$. Interestingly, we found upregulation of several EMT suppressors, such as GRHL2 (refs. ${ }^{49,50}$ ), Ovol2 (ref. $\left.{ }^{51}\right)$, Pofut2 $\left(\right.$ ref. $^{52}$ ) and Elf3 (ref. ${ }^{53}$ ) in endoderm compared

Fig. 2 | Mesoderm and endoderm form by distinct molecular programs. a, Schematic of FACS of early-, mid- and late-streak-stage FVF embryos for scRNA-seq analysis ( $n=79$ for early- to mid-streak-stage embryos and $n=24$ for mid- to late-streak-stage embryos). b, UMAP plot with RNA velocity arrows, coloured by CellRank's metastable state assignment. Each shown tissue is either in the initial (epiblast (Epi)), intermediate (posterior epiblast (pEpi)) or final state (AME, definitive endoderm, lateral plate mesoderm (LPM) and nascent endothelium (NE)). c, UMAP showing CellRank's fate probabilities for different tissues as pie charts $(n=9,794$ cells). The partitions of each pie chart show the previously identified initial, intermediate or final state. Dashed lines indicate significant connections between clusters (PAGA graph model). Arrows indicate consistent RNA velocity between two clusters. The thickness of each line shows the confidence of the model. The solid line without an arrowhead suggests a transition along the velocity between clusters but not unique flow. IM, intermediate mesoderm; PGC, primordial germ cell; PM, paraxial mesoderm. d, Scatter plot of lineage drivers, showing the correlation of gene expression for the lineages definitive endoderm and LPM, computed using CellRank. The top 50 correlated genes are indicated by dashed horizontal and vertical lines. e, Stacked violin plots showing the gene expression distribution (columns) with definitive endoderm, EMT, EMT inhibitors and cell adhesion genes of all tissues (rows) ( $n=2,215$ (posterior epiblast); $n=2,198$ (paraxial mesoderm); $n=1,183$ (LPM); $n=701$ (definitive endoderm); $n=389$ (intermediate mesoderm); $n=350$ (transitory progenitors); $n=278$ (AME); $n=91$ (nascent endothelium)). The colours correspond to normalized median gene expression for each group. f, UMAP plots coloured by the log[counts per million +1] normalized gene expression. $\mathbf{g}$, Schematic of endoderm and mesoderm differentiation of $\mathrm{T}^{\mathrm{GFP} /+}$; Foxa2 ${ }^{\mathrm{tagRFP} /+} \mathrm{mESC}$. $\mathbf{h}$, Heatmap of FACS-sorted endodermal and mesodermal subpopulations expressing different levels of CD24 at days 2 and 4, showing RNA expression levels of pluripotency, endoderm, mesoderm and EMT genes in mESCs (ES), early (definitive endoderm progenitor (DEP)/mesoderm progenitor (MP)) and late (definitive endoderm (DE)/mesoderm (MES)) endoderm and mesoderm cells. The coloured boxes indicate differentially expressed genes (DEGs) in DEP versus mesoderm progenitor (green and orange, respectively) or definitive endoderm versus mesoderm (blue and red, respectively) and whether Foxa2 binds (pink) or binds and regulates them (purple). 
with mesoderm (Fig. 5c). Furthermore, ChIP-seq data analysis suggested that Ovol2 is bound and potentially regulated by Foxa2 (Fig. $5 \mathrm{c}$ and Supplementary Table 1). Analysis of RNA-seq data from
Foxa $2^{\text {Venus/+ }}$ and Foxa2 ${ }^{\text {Venus/Venus }}$ mESC differentiations revealed that these EMT inhibitors are downregulated upon loss of Foxa2 (Fig. 4b). Altogether, these findings show that Foxa2 not only counteracts a a

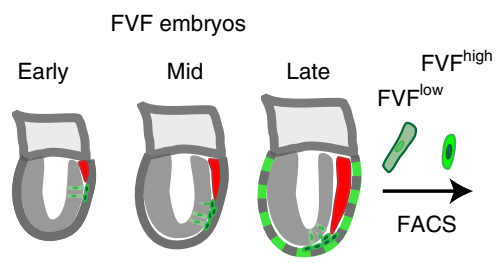

d

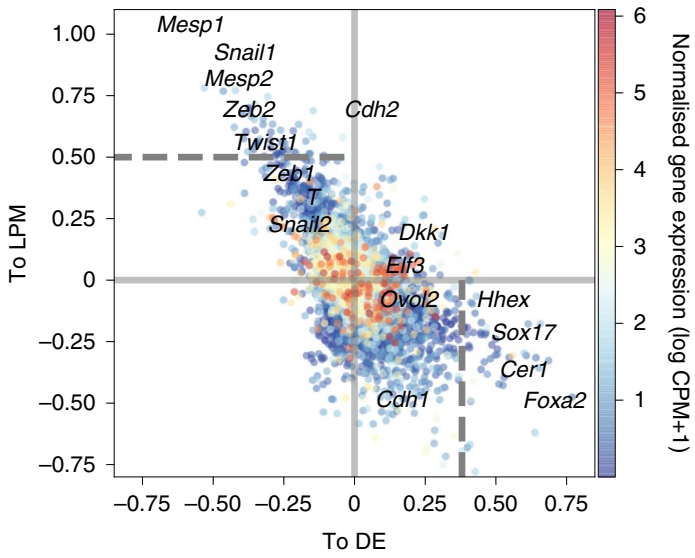

f
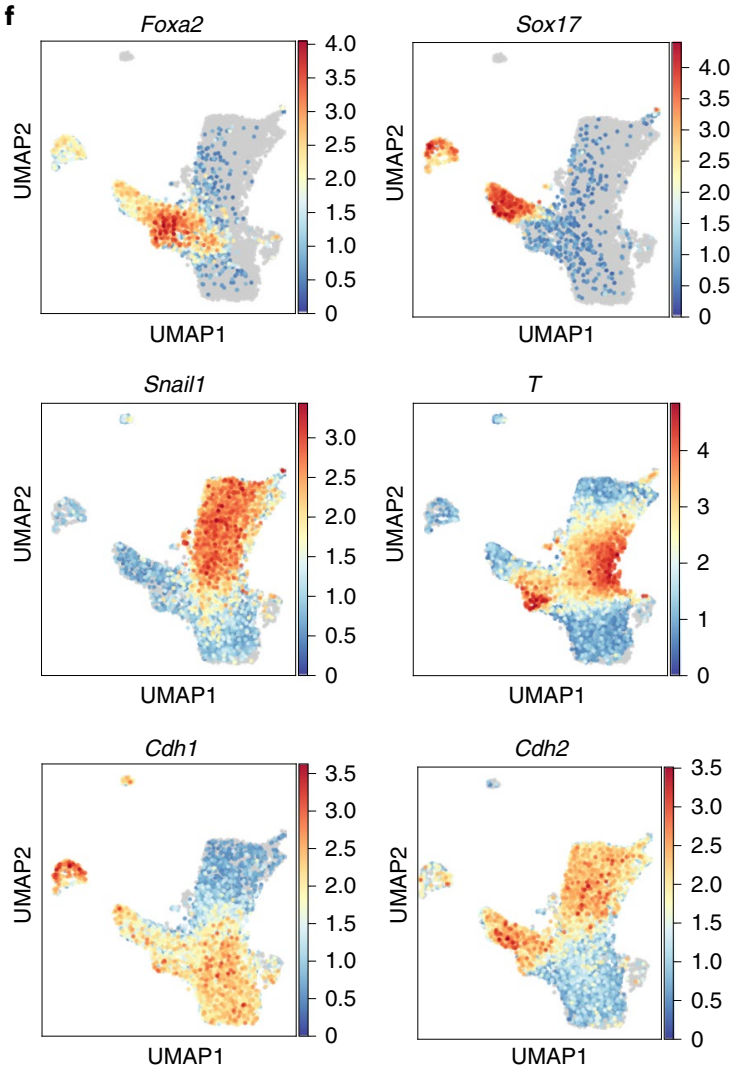

e b

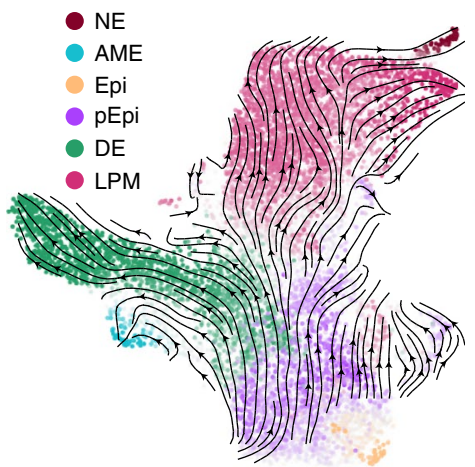

c

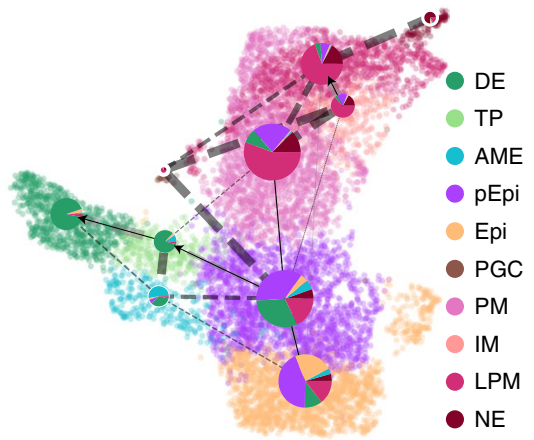

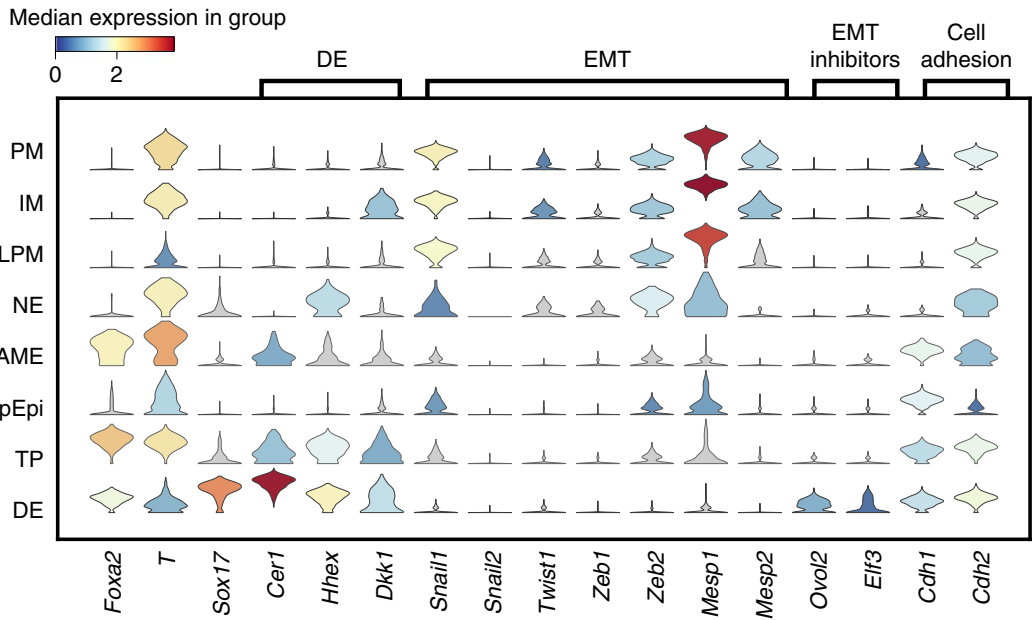

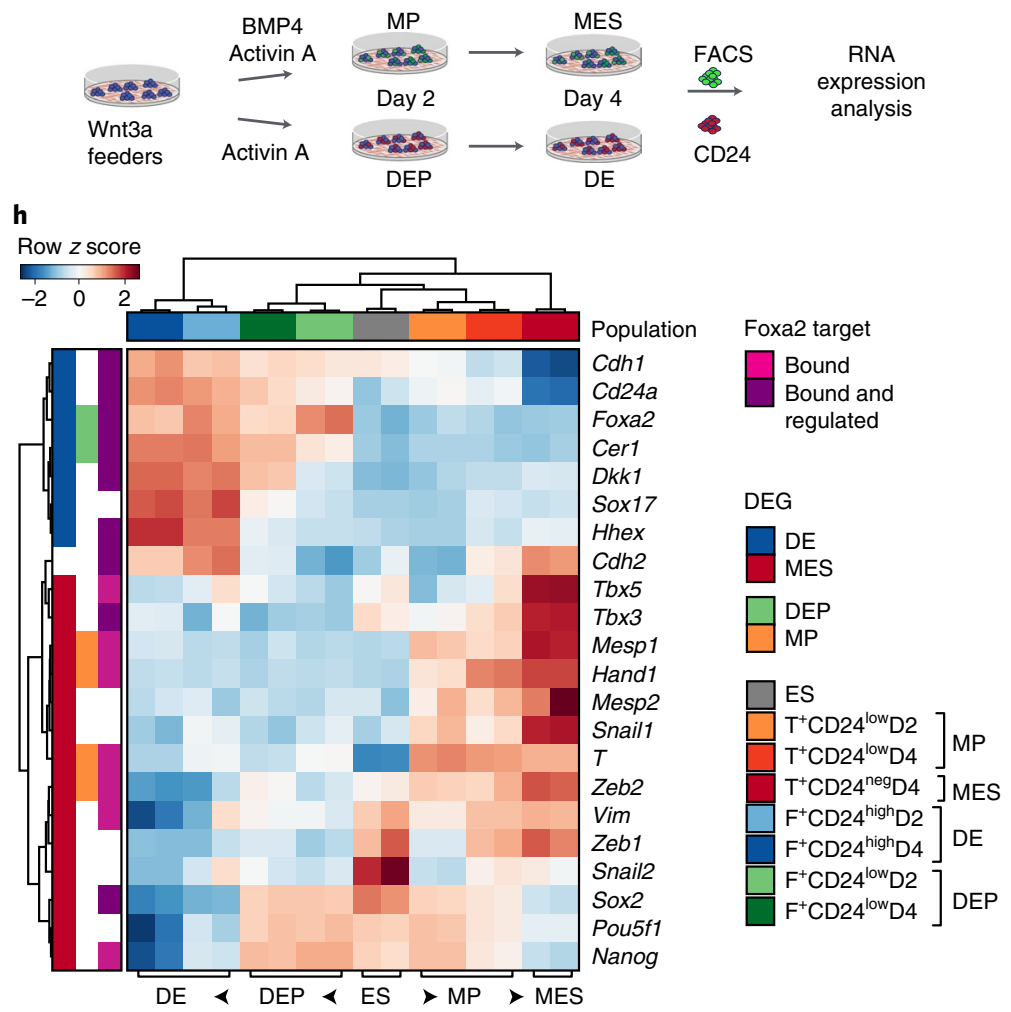




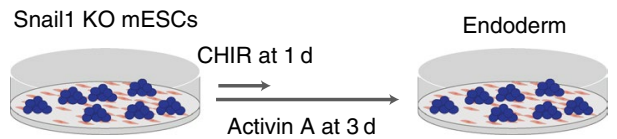

d

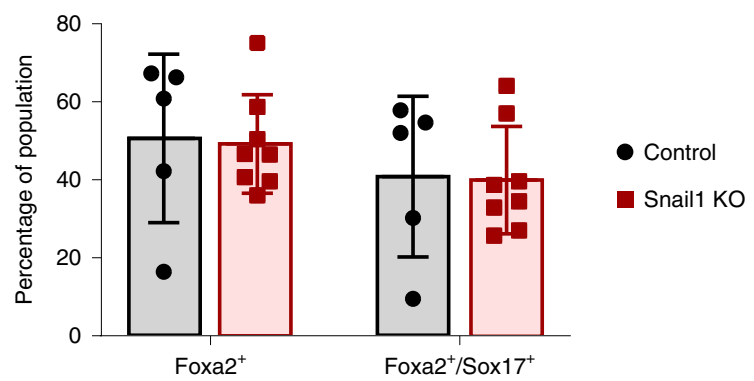

e

4n mT $\leftrightarrow$ Snail1 KO mESC

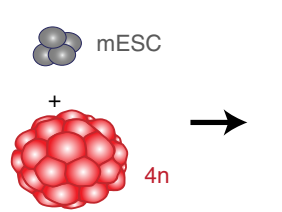

LB
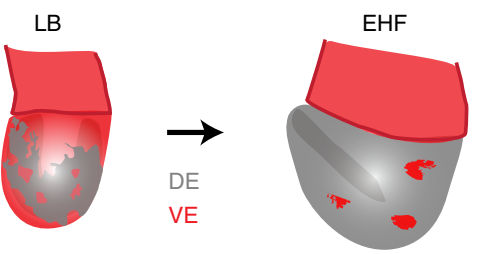

h

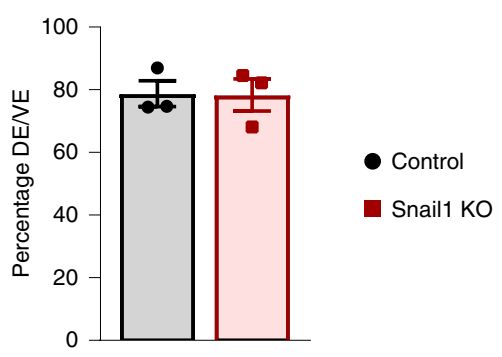

b

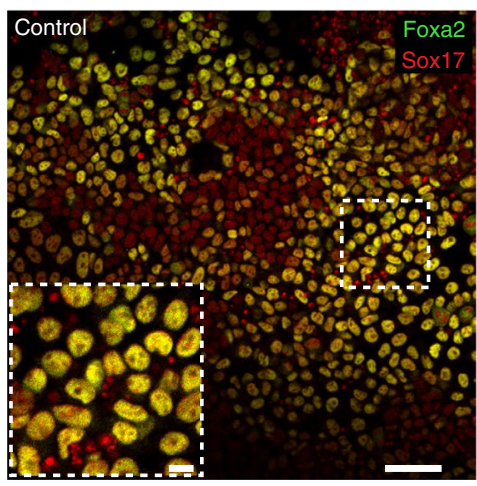

c

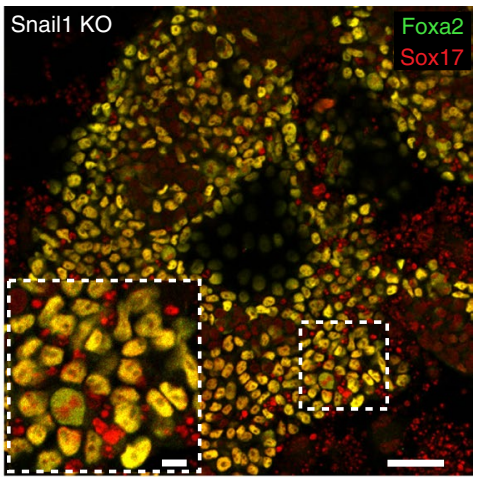

f
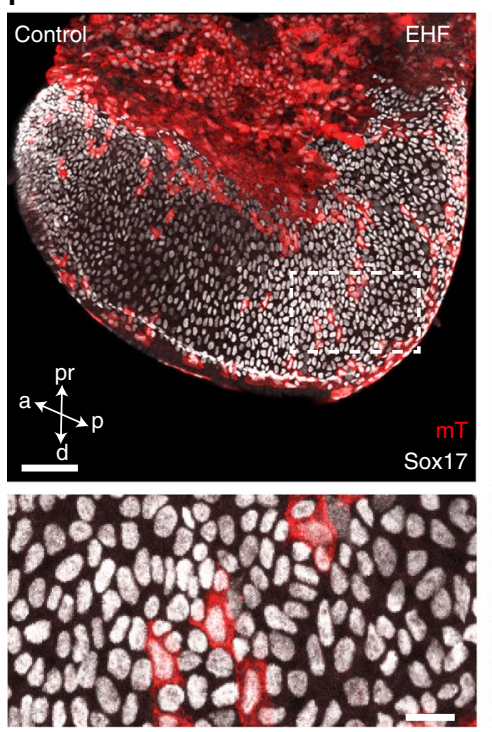

g
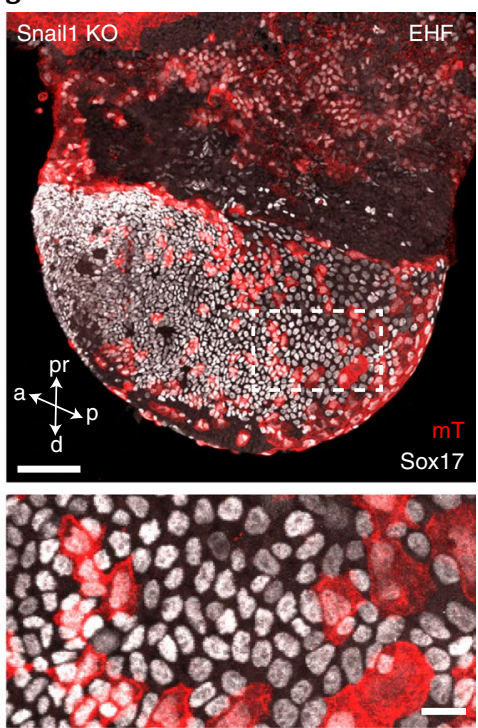

Fig. 3 | Snail1 is not required for endoderm formation. a, Endoderm differentiation schematic of Snail1 knockout mESCs. b,c, Immunohistochemistry of differentiated wild-type (control) (b) and Snail1 knockout (c) endoderm cells at day 3 stained for Sox17 and Foxa2. d, FACS quantification of Foxa2 ${ }^{+}$and Sox $17^{+} /$Foxa $2^{+}$cells in control versus Snail 1 knockout endoderm differentiations (two-tailed unpaired Student's $t$-test (no significant difference); $n=5$ (control); $n=8$ (Snail1 knockout)). e, Schematic of the generation of tetraploid aggregation chimeras with Snail1 knockout mESCs. EHF, early headfold stage. LB, late bud stage. 4 n, tetraploid. $\mathbf{f}, \mathbf{g}$, Maximum projection from confocal images of wild-type (control) (f) and Snail1 knockout chimeric embryos (g) stained for RFP (mT) and Sox17, showing the dispersal of visceral endoderm $\left(\mathrm{mT}^{+}\right)$by Snail1 mutant or wild-type definitive endoderm cells. The bottom images show magnified views of the areas highlighted by dashed white rectangles in the images above. $\mathbf{h}$, Quantification of formed definitive endoderm over visceral endoderm in wild-type (control) and Snail1 mutant embryos at the early headfold stage (two-sided unpaired Student's $t$-test (no significant difference); $n=3$ embryos each). All samples were derived from biologically independent experiments. The images in $\mathbf{b}$ and $\mathbf{c}$ are representative of five independent differentiations each. The images in $\mathbf{f}$ and $\mathbf{g}$ are representative of three embryos each. The data are presented as mean values \pm s.e.m. Scale bars, $50 \mu \mathrm{m}$ (insets, $10 \mu \mathrm{m}$ ).

complete EMT in endoderm progenitors and the definitive endoderm lineage by the induction of Wnt inhibitors and EMT suppressors, but also quickly re-establishes an epithelial identity by the activation of target genes that regulate cell polarity and adhesion ${ }^{11}$. Thus, Foxa2 serves as an epithelial gatekeeper and EMT suppressor during endoderm formation.

Dynamic molecular changes drive endoderm differentiation. Finally, to understand how the definitive endoderm is formed on the morphogenetic level, we analysed and verified our time-resolved and lineage-mapped scRNA-seq data. We first compared differences of the anterior versus posterior epiblast epithelium. This disclosed lower expression of apical-basal polarity (Crb3, Ezr and EBP50) and tight junction genes (Cldn6 and 7) (Extended Data Fig. 7a,b). Both adherens junction E-cadherin and the tight junction protein Claudin7 were less abundant in posterior epiblast cells, and Claudin7 appeared in the cytoplasm and less at the plasma membrane (Fig. 7a,b and Extended Data Fig. 7c). Next, we compared Foxa2 ${ }^{-}$with Foxa2 ${ }^{+}$posterior epiblast cells (Extended Data Fig. 7a,d). Foxa2 ${ }^{+}$posterior epiblast cells showed a distinct transcription factor gene expression profile (Foxa2, Eomes, Mixl1, Lhx1 and $G s c$ ), but also particularly high expression of metalloproteinase genes (Adamts9, Adam19 and Mmp14) involved in basement membrane remodelling and morphogenetic dynamics during gastrulation $^{54}$. Interestingly, the high expression of metalloproteinases was maintained in Foxa $2^{\text {high }}$ transitory progenitors (Fig. 7) and correlated with basement membrane remodelling in Foxa2 ${ }^{+}$posterior epiblasts (Fig. 7 and Extended Data Fig. 7e-g). Interestingly, we observed a transient downregulation of apical-basal polarity (Ezr, Crb3, EBP50, Pard6b and Scribble) and tight junction genes (Cldn6 and 7 ) and proteins in the endoderm transition from Foxa $2^{\text {low }}$ posterior epiblast to Foxa $2^{\text {high }}$ transitory progenitors and definitive 

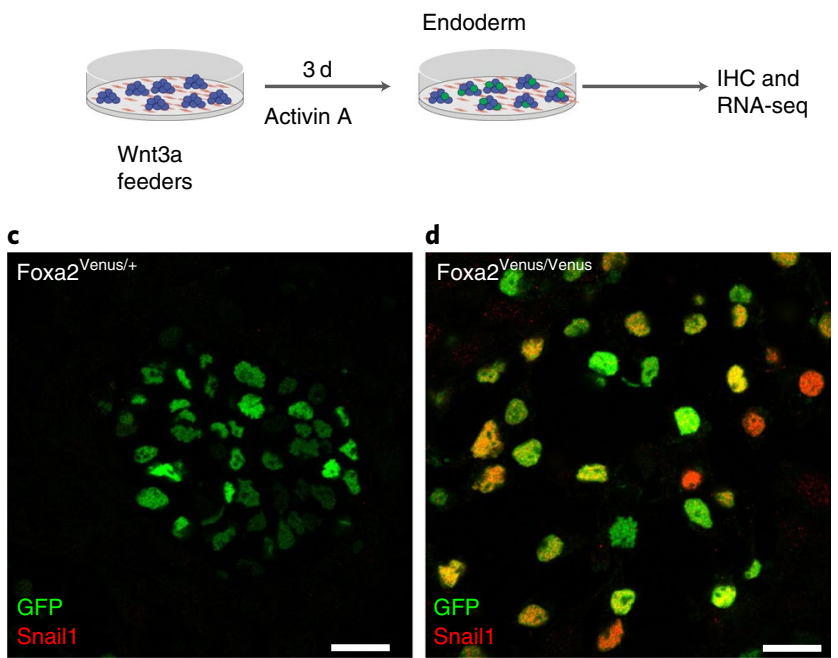

b

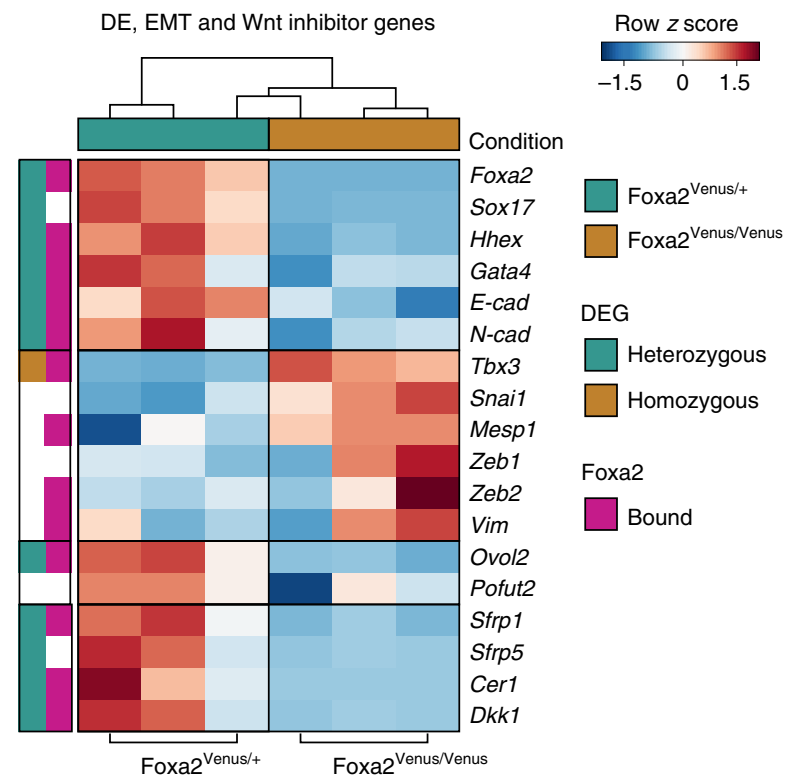
4n CD1 WT $\leftrightarrow$ Foxa2 ${ }^{\text {Venus/Venus }}$ KO mESC

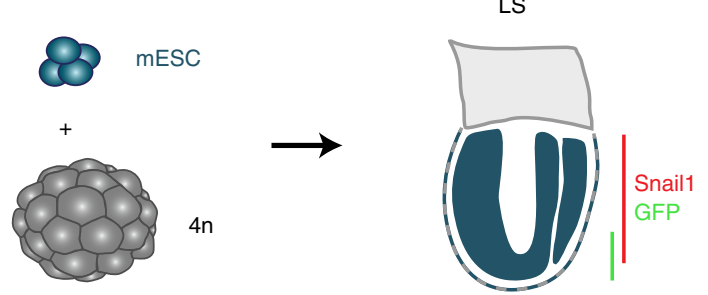

$\mathbf{h}$

- Foxa2 $2^{\text {Venus high }} /$ Fnail1 $^{-} \quad$ Foxa2 ${ }^{\text {Venus high }} /$ Snail1 $^{+}$

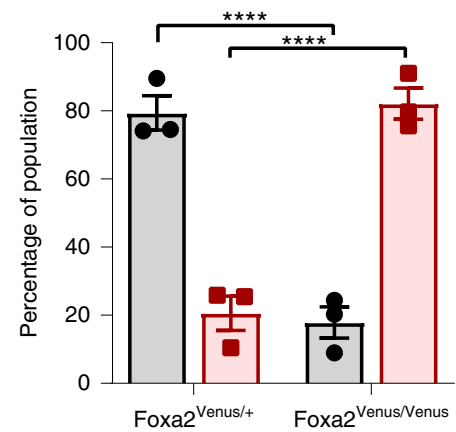

f
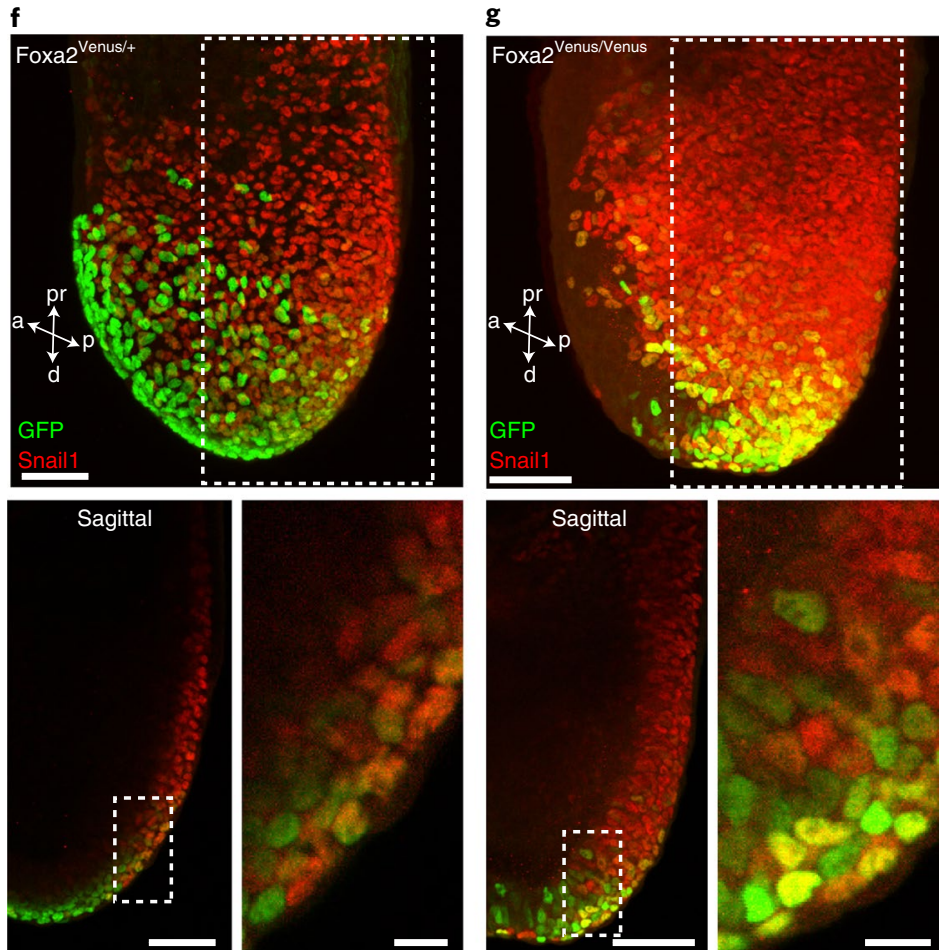

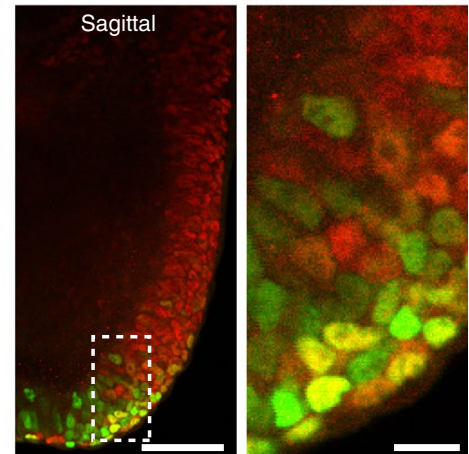

Fig. 4 | Foxa2 suppresses Snail1 in the endoderm lineage. a, Schematic of endoderm differentiation of Foxa2 ${ }^{\text {venus/venus }}$ knockout $m E S C s$. IHC, immunohistochemistry. b. Heatmap of Foxa2 $2^{\text {Venus/+ }}$ and Foxa2 $2^{\text {Venus/Venus }}$ endoderm ${ }^{34}$ showing the up- and downregulation of endoderm genes, EMT activators, inhibitors and Wnt signalling inhibitors ( $n=3$ independent differentiations). The pink boxes indicate genes bound by Foxa2. The green and brown boxes mark genes with significantly different expression in Foxa2 $2^{\text {Venus/+ }}$ and Foxa2 $2^{\text {Venus/Venus }}$ cells, respectively. c,d, Immunostainings against Venus and Snail1 of Foxa2 ${ }^{\text {Venus/+ }}$ control (c) and Foxa2 ${ }^{\text {Venus/Venus }}$ knockout mESCs (d) differentiated under endoderm conditions for 3 d. e, Schematic of aggregation chimeras generated with Foxa2 ${ }^{\text {Venus/+ }}$ and Foxa2 ${ }^{\text {Venus/Venus }} \mathrm{mESC}$. LS, late-streak stage; WT, wild type. $\mathbf{f}, \mathbf{g}$, Maximum projections (top) and single optical sections

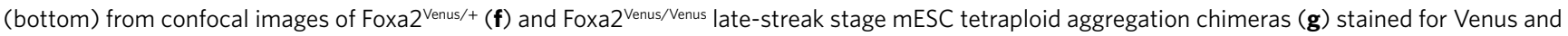
Snail1. h, Quantification of Venus high and Snail1+ ${ }^{+}$or Snail1- cells in Foxa2 ${ }^{\text {Venus/+ }}$ and Foxa2 ${ }^{\text {Venus/Venus }}$ aggregation chimeras ( ${ }^{\star \star \star \star *} P<0.0001$; ordinary one-way analysis of variance with Tukey's multiple comparisons test; $n=3$ embryos). Statistically non-significant results are not indicated in the figure. All samples were derived from biologically independent experiments. The images in $\mathbf{c}$ and $\mathbf{d}$ are representative of four independent endoderm differentiations. The images in $\mathbf{f}$ and $\mathbf{g}$ are representative of three embryos each. The data are presented as mean values \pm s.e.m. Scale bars, $50 \mu \mathrm{m}$ (insets, $10 \mu \mathrm{m}$ ).

endoderm (Fig. 7 and Extended Data Fig. 7h), probably due to a lack of basement membrane polarity cues. Altogether, the transition of columnar Foxa $2^{\text {low }}$ posterior epiblast through Foxa $2^{\text {high }}$ transitory progenitors to squamous Foxa $2^{\text {high }} /$ Sox $17^{+}$definitive endoderm shows several attributes of an epithelial cell plasticity program, including simultaneous expression of E- and N-cadherin and transient downregulation of polarity and adherens junction and tight junction proteins, best defined by a partial $\mathrm{EMT}^{20,55}$. 
a

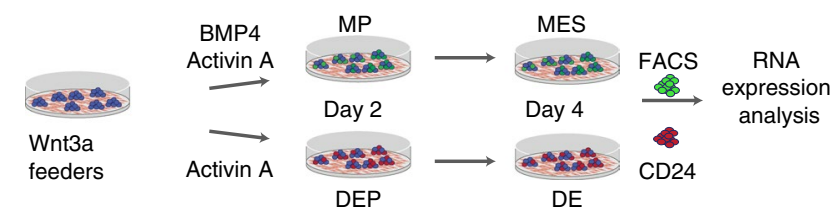

c
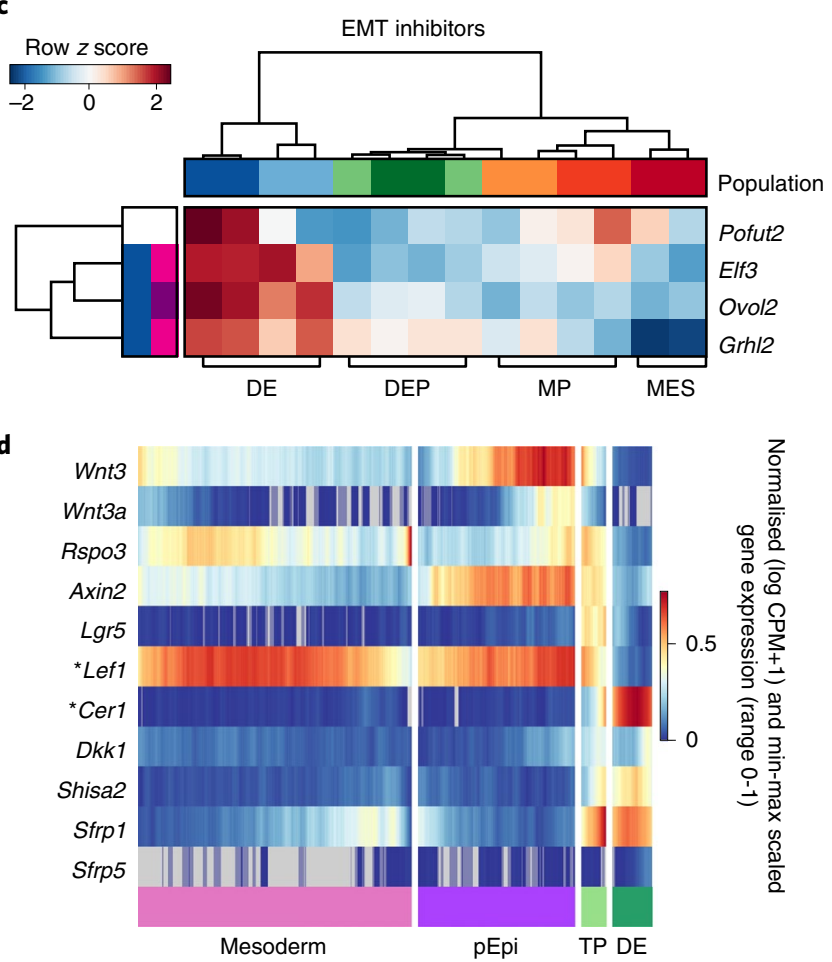

b

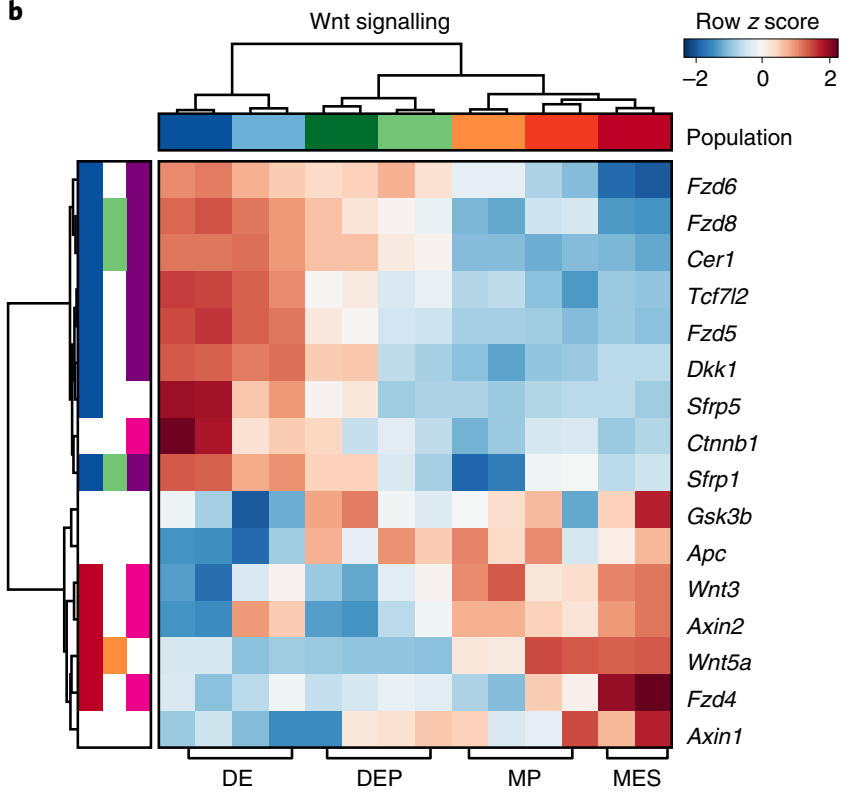

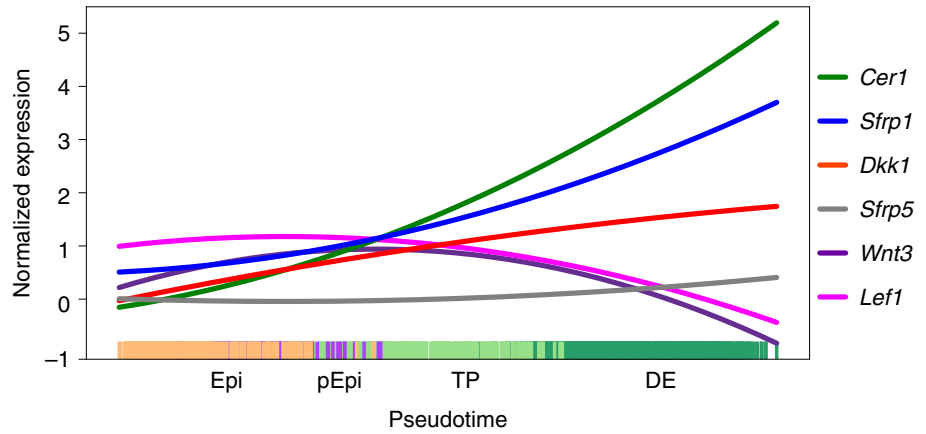

g
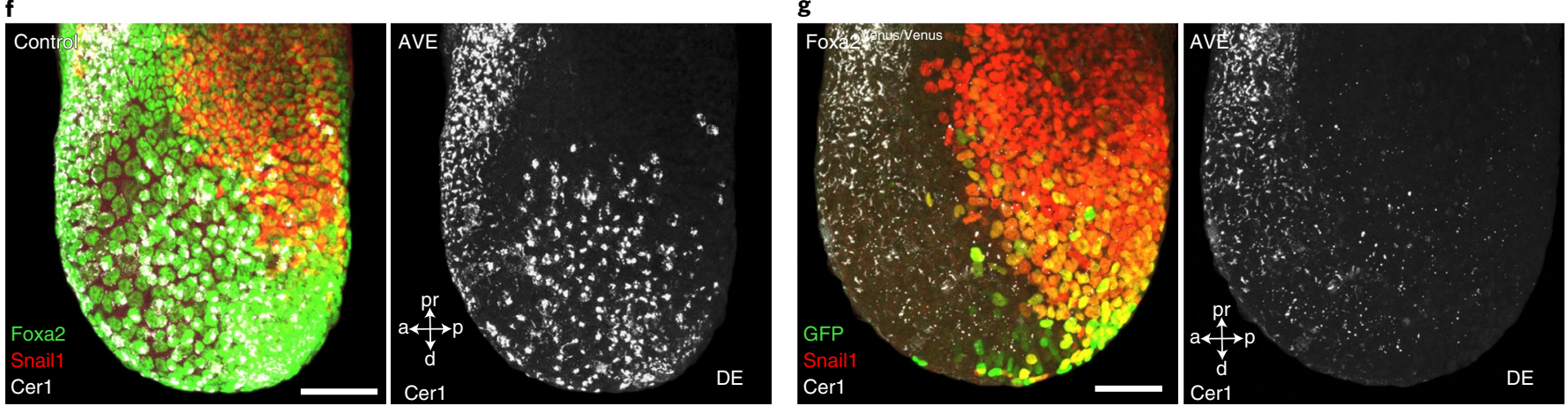

Fig. 5 | Foxa2 activates Wnt inhibitors in endoderm. a, Schematic of endoderm and mesoderm differentiation of TGFP/+; Foxa2 ${ }^{\text {tagRFP/+ }}$ mESCs. b, Heatmap of FACS-sorted endodermal and mesodermal subpopulations expressing different levels of CD24 at days 2 and 4 , showing RNA expression levels of canonical Wnt signalling genes. c, Heatmap of endoderm and mesoderm from differentiations of T TFP/+; Foxa2tagRFP/+ cells, showing upregulation of EMT suppressors in endoderm. In $\mathbf{b}$ and $\mathbf{c}$, the coloured boxes to the left indicate genes that were differentially expressed in DEP versus mesoderm progenitor (green and orange, respectively) or definitive endoderm versus mesoderm (blue and red, respectively) and whether Foxa2 binds (pink) or binds and regulates them (purple). d, Clustered heatmap showing the smoothed (sliding window of $n=100$ cells) and scaled gene expression of Wnt signalling genes in mesoderm (paraxial mesoderm, intermediate mesoderm, LPM and nascent endothelium), posterior epiblast, transitory progenitors and definitive endoderm. e, Quadratic spline plots showing genes involved in activation and inhibition of Wnt signalling along diffusion pseudotime from epiblasts to posterior epiblasts to transitory progenitors to definitive endoderm. $\mathbf{f}, \mathbf{g}$, Maximum projections of control (representative of six embryos) (f) and Foxa2 ${ }^{\text {Venus/Venus }}$ knockout (representative of eight embryos) (g) late-streak-stage aggregation embryos immunostained for GFP or Foxa2, Snail1 and Cer1. All samples were derived from biologically independent experiments. All shown confocal images are single planes of a z stack unless otherwise stated. Scale bars, $50 \mu \mathrm{m}$. 

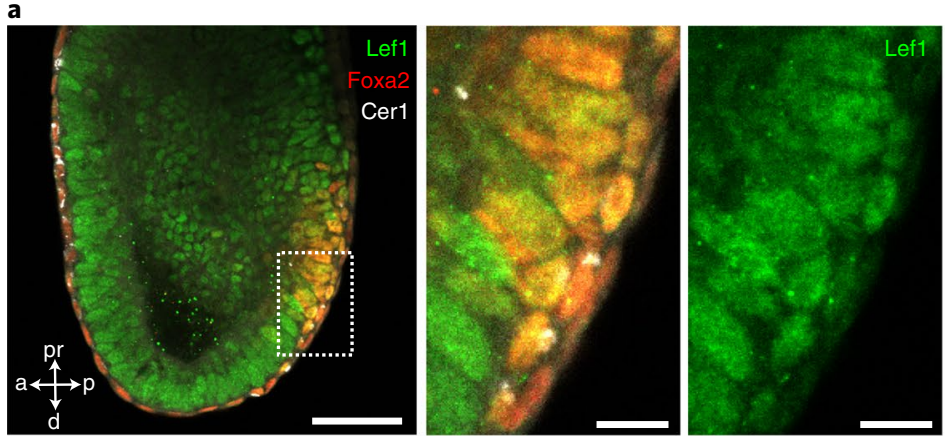

c
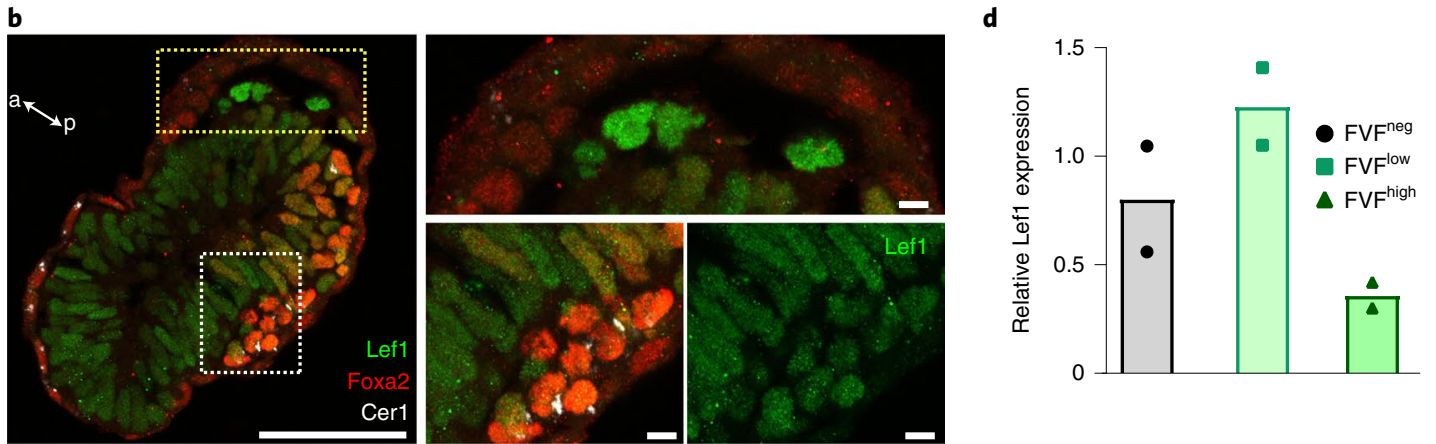

e

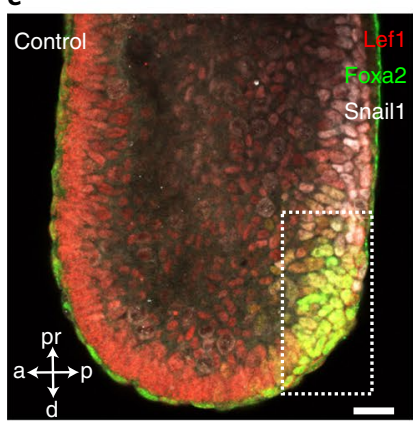

f

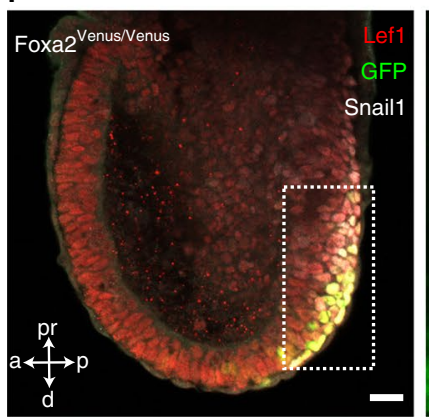

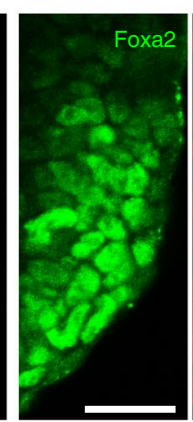
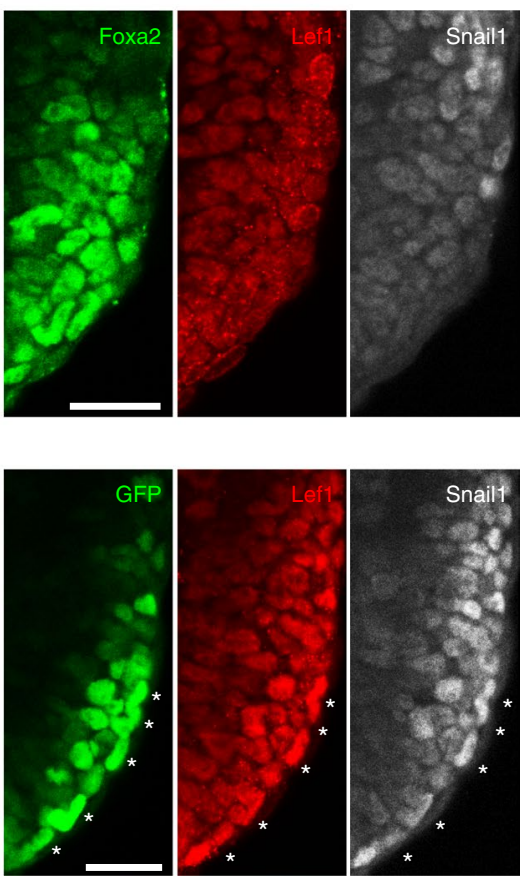

g
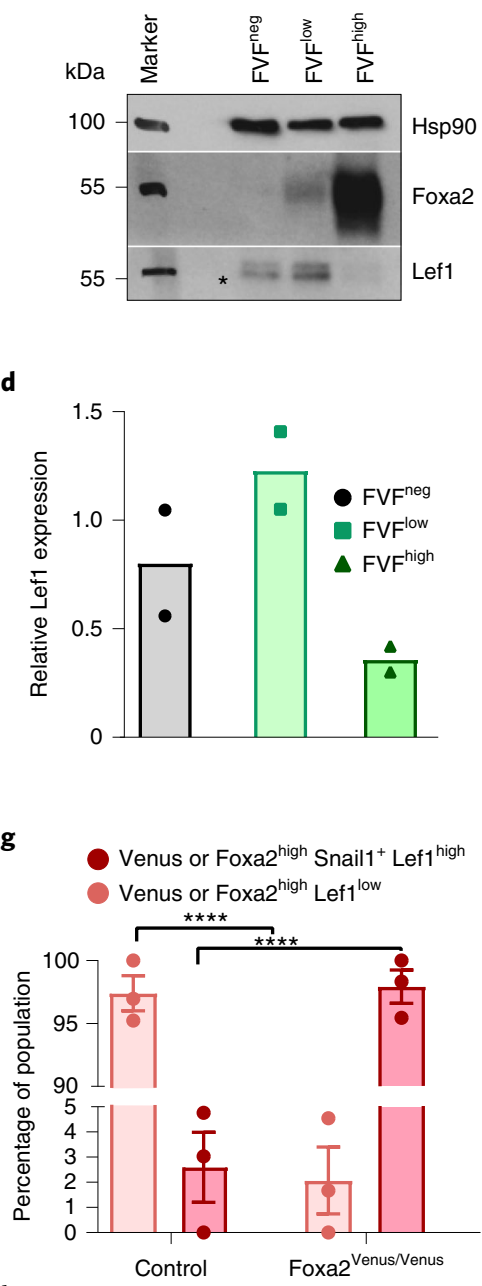

h

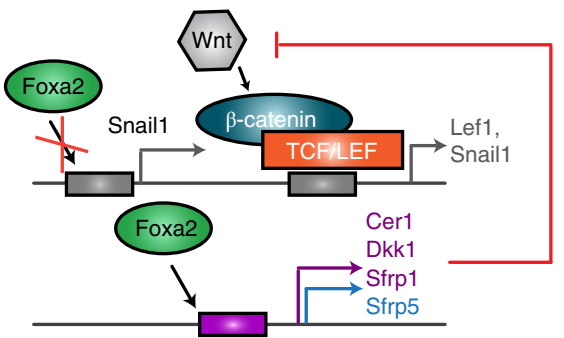

Fig. 6 | Foxa2 indirectly suppresses Wnt signalling in endoderm. a,b, Confocal image of a mid-streak-stage embryo (a) and a transverse section through the epiblast of a mid-streak-stage embryo (b) immunostained for Foxa2, Lef1 and Cer1. c,d, Western blot analysis (c) and quantification (d) of Lef1 from FACS-sorted FVFneg, FVFlow and FVFigh cells of $n=36$ and $n=122$ FVF embryos ( $n=2$ experiments). The asterisk marks unspecific bands. e,f, Representative confocal images of control (e) and Foxa2 ${ }^{\text {Venus/Venus }}$ knockout late-streak-stage aggregation chimeras (f) immunostained for Foxa2/GFP, Lef1 and Snail1. g, Quantification of Venus or Foxa $2^{\text {high }}$ cells colocalizing with either Snail1 and Lef $1^{\text {high }}$ or Lef1 ${ }^{\text {low }}$ in control versus Foxa2 knockout aggregation embryos ( ${ }^{\star \star \star \star} P<0.0001$; ordinary one-way analysis of variance with Tukey's multiple comparison test; $n=3$ embryos). The data are presented as mean values \pm s.e.m. Statistically non-significant results are not indicated in the figure. $\mathbf{h}$, Schematic illustrating how Foxa2 inhibits a full EMT in endoderm. Foxa2 directly (purple) or indirectly (blue) controls the expression of Wnt inhibitors. The purple and grey boxes represent the transcription factor-binding sites of gene specific promoters. TCF/LEF, T-cell factor/lymphoid enhancer factor. All samples were derived from biologically independent experiments. The images in $\mathbf{a}, \mathbf{b}, \mathbf{e}$ and $\mathbf{f}$ are representative of six, three, three and three embryos, respectively. All shown confocal images are single planes of a $z$ stack unless otherwise stated. Scale bars, $50 \mu \mathrm{m}$ (insets, $10 \mu \mathrm{m}$ ). 

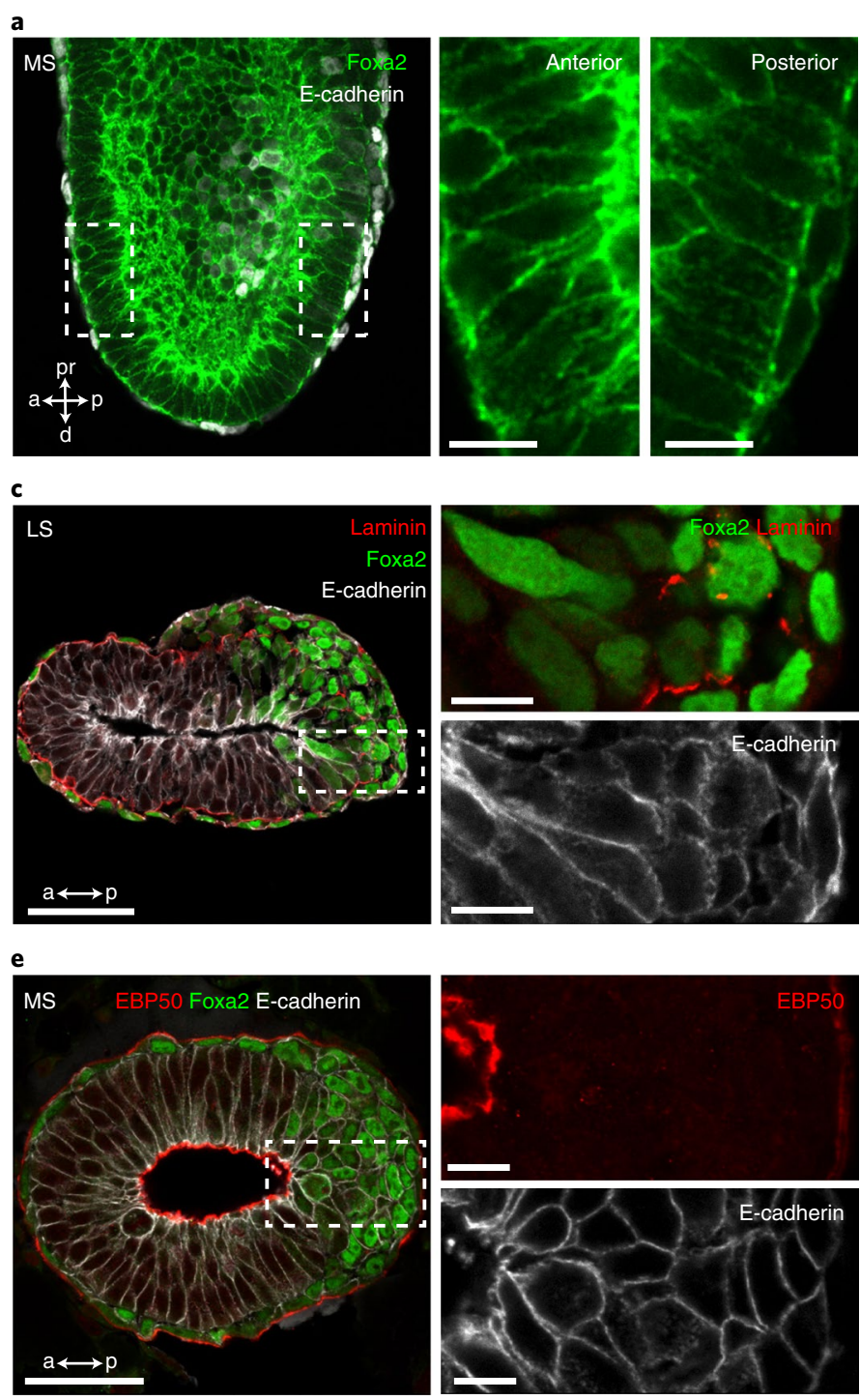

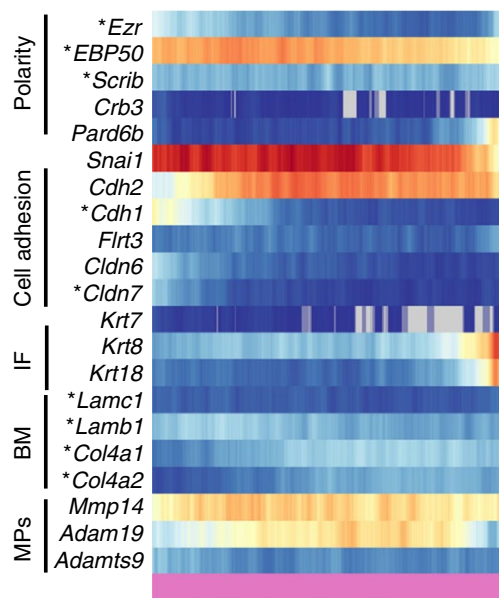

Mesoderm

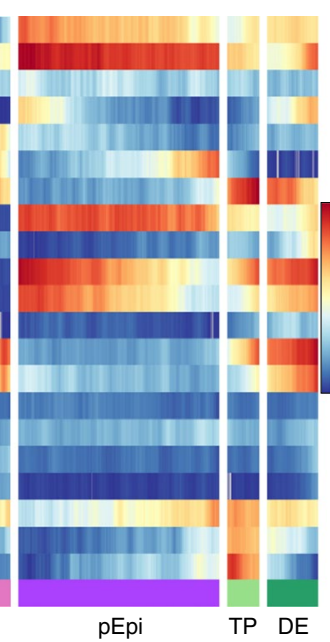

\section{Discussion}

Here, we present a revised concept of germ layer formation during gastrulation (Fig. 7h). Before gastrulation is initiated, $\mathrm{T}^{+}$mesoderm progenitors and Foxa $2^{\text {low }}$ epiblast progenitors are already specified b
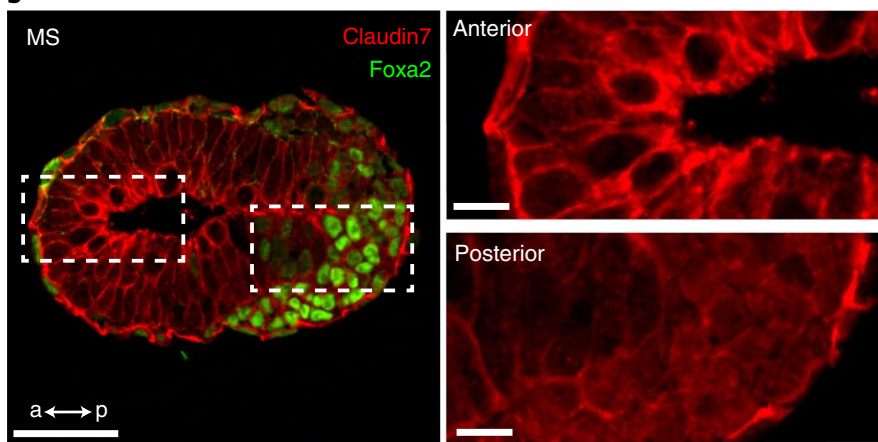

Posterior

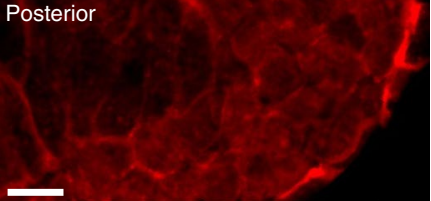

d
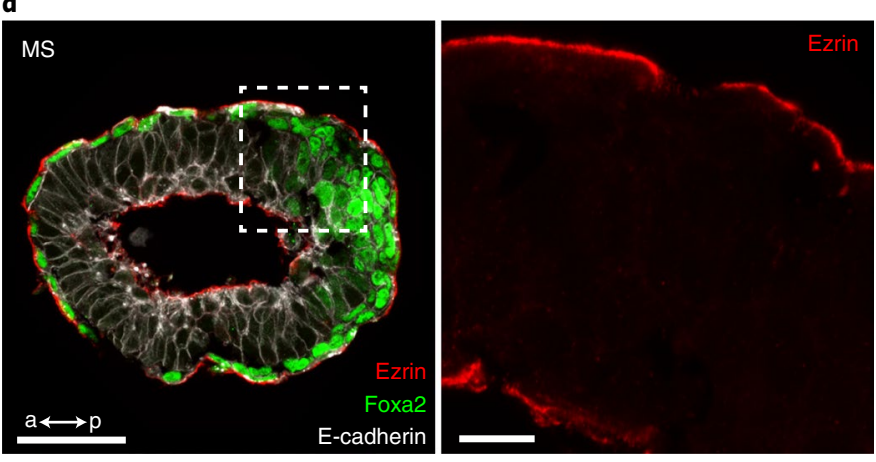

g

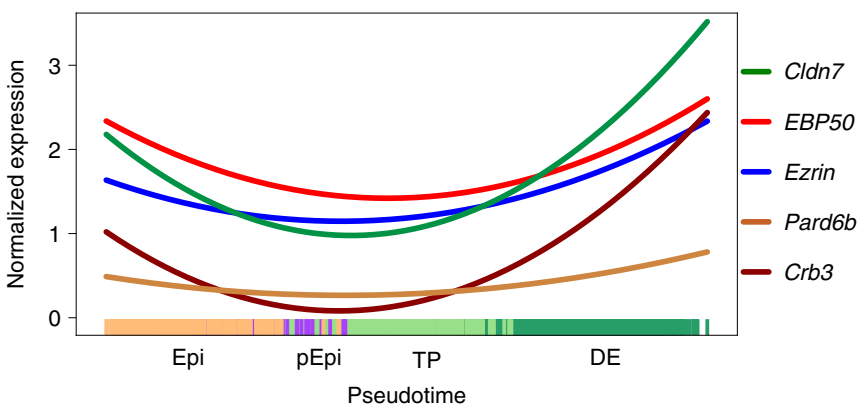

h $\begin{array}{ll}\text { pr Lefty1 } & \text { Wnt3 } \\ a & \text { Dkk1 }\end{array}$

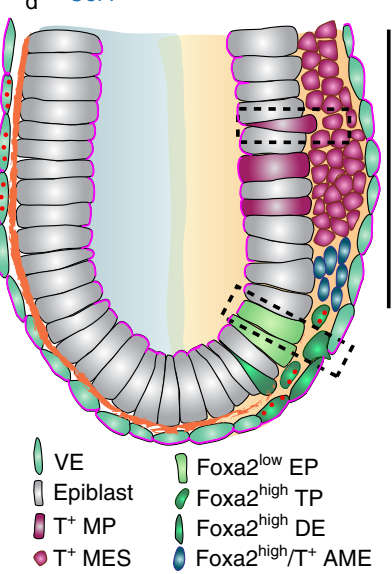

in the posterior epiblast. Mesoderm progenitors ingress into the primitive streak by a complete EMT to commit to a mesenchymal fate. In contrast, Foxa $2^{+}$posterior epiblasts and Foxa2 $2^{\text {high }}$ transitory progenitors leave the posterior epiblast epithelium by transient 
Fig. 7 | Epithelial cell plasticity drives endoderm formation. a, Representative image of a mid-streak-stage embryo stained for Foxa2 and E-cadherin. b-e, Transverse sections through the epiblasts of wild-type mid- and late-streak-stage embryos immunostained for Foxa2 and Claudin7 (b), Foxa2, laminin and E-cadherin (c), Foxa2, Ezrin and E-cadherin (maximum projection) (d) and Foxa2, EBP50 and E-cadherin (e). f, Clustered heatmap showing the smoothed (sliding window of $n=100$ cells) and scaled gene expression of polarity, cell adhesion, intermediate filaments (IF), basement membrane (BM) and metalloproteinases (MPs) in mesoderm (paraxial mesoderm, intermediate mesoderm, LPM and nascent endothelium), posterior epiblasts, transitory progenitors and definitive endoderm. The asterisks mark genes that have been confirmed by immunohistochemistry. $\mathbf{g}$, Quadratic spline plot showing the expression of polarity and cell adhesion genes along diffusion pseudotime from epiblast to posterior epiblast to transitory progenitors to definitive endoderm. $\mathbf{h}$, Schematic of endoderm formation by partial EMT. All samples were derived from biologically independent experiments. The images in a-d are representative of three embryos each. All shown confocal images are single planes of a z stack unless otherwise stated. Scale bars, $50 \mu \mathrm{m}$ (insets, $10 \mu \mathrm{m}$ ).

upregulation of metalloproteinases, remodelling of the basement membrane and a transient downregulation of apical-basal polarity genes and proteins-features reminiscent of a partial EMT program. During this process, we observed an absence of EMT transcription factors, maintained E-cadherin expression, activation of EMT suppressors and expression of adherens junction and tight junction genes and proteins $\mathrm{s}^{12,13,24-28}$. The co-expression of $\mathrm{E}$ - and $\mathrm{N}$-cadherin in the nascent definitive endoderm and visceral endoderm probably increases the segregation of the endoderm from the mesoderm germ layer by differential cell adhesion, as proposed by Townes and Holtfreter in $1955^{56}$. Previously, we have shown that Foxa $2^{\text {high }}$ transitory progenitors re-establish apical-basal polarity, adherens junctions and tight junctions while they start to express Sox17 and intercalate into the overlying visceral endoderm layer ${ }^{11,29,30}$.

Importantly, Foxa2 acts as an epithelial gatekeeper and EMT suppressor to protect endoderm progenitors from undergoing a complete mesenchymal transition. Foxa2 is necessary to prevent EMT transcription factor activation via the activation of canonical Wnt $/ \beta$-catenin signalling inhibitors. Thus, these results are not only important to understand basic mechanisms of gastrulation, but also have broader implications, as EMT causes detrimental cancer metastasis. Importantly, endoderm-derived lung, pancreatic and colorectal cancers are among the most common and deadliest cancers worldwide (National Cancer Institute). So far, cancer metastasis was always associated with EMT but recent studies contradict the necessity of a complete EMT-MET cycle for the dissemination of cancer cells and for invasion and metastasis in pancreatic cancer ${ }^{55,57,58}$. Mechanisms of epithelial cell plasticity might allow cancer cell dissemination and metastasis and require further in-depth mechanistic studies to provide alternative targets for therapeutic intervention. In line with our basic developmental biology findings, it was shown that Foxa2 antagonizes a full EMT process in pancreatic and lung cancer ${ }^{59-61}$. Foxa2 is an EMT suppressor but also an epithelial gatekeeper; thus, maintaining FOXA2 expression during cancer initiation and progression will prevent a complete EMT-MET cycle and eventually cancer dissemination.

\section{Online content}

Any methods, additional references, Nature Research reporting summaries, source data, extended data, supplementary information, acknowledgements, peer review information; details of author contributions and competing interests; and statements of data and code availability are available at https://doi.org/10.1038/ s41556-021-00694-X.

Received: 2 March 2021; Accepted: 5 May 2021;

Published online: 24 June 2021

\section{References}

1. Argelaguet, R. et al. Multi-omics profiling of mouse gastrulation at single-cell resolution. Nature 576, 487-491 (2019).

2. Pijuan-Sala, B. et al. A single-cell molecular map of mouse gastrulation and early organogenesis. Nature 566, 490-495 (2019).
3. Nowotschin, S. et al. The emergent landscape of the mouse gut endoderm at single-cell resolution. Nature 569, 361-367 (2019).

4. Lim, J. \& Thiery, J. P.Epithelial-mesenchymal transitions: insights from development. Development 139, 3471-3486 (2012).

5. Nieto, M. A., Huang, R. Y.-J., Jackson, R. A. \& Thiery, J. P.EMT: 2016. Cell 166, 21-45 (2016).

6. Thiery, J. P., Acloque, H., Huang, R. Y. J. \& Nieto, M. A.Epithelialmesenchymal transitions in development and disease. Cell 139, 871-890 (2009)

7. Zorn, A. M. \& Wells, J. M. Vertebrate endoderm development and organ formation. Annu. Rev. Cell Dev. Biol. 25, 221-251 (2009).

8. Wilkinson, D. G., Bhatt, S. \& Herrmann, B. G.Expression pattern of the mouse $\mathrm{T}$ gene and its role in mesoderm formation. Nature 343, 657-659 (1990)

9. Ang, S. L. \& Rossant, J. HNF-3 $\beta$ is essential for node and notochord formation in mouse development. Cell 78, 561-574 (1994).

10. Weinstein, D. C. et al. The winged-helix transcription factor HNF-3 $\beta$ is required for notochord development in the mouse embryo. Cell 78, 575-588 (1994)

11. Burtscher, I. \& Lickert, H.Foxa2 regulates polarity and epithelialization in the endoderm germ layer of the mouse embryo. Development 136, 1029-1038 (2009).

12. Arnold, S. J. \& Robertson, E. J.Making a commitment: cell lineage allocation and axis patterning in the early mouse embryo. Nat. Rev. Mol. Cell Biol. 10, 91-103 (2009).

13. Nowotschin, S., Hadjantonakis, A.-K. K. \& Campbell, K.The endoderm a divergent cell lineage with many commonalities. Development 146, dev150920 (2019).

14. Lindsley, R. C. et al. Mesp1 coordinately regulates cardiovascular fate restriction and epithelial-mesenchymal transition in differentiating ESCs. Cell Stem Cell 3, 55-68 (2008).

15. TextXKitajima, S., Takagi, A., Inoue, T. \& Saga, Y.MesP1 and MesP2 are essential for the development of cardiac mesoderm. Development 127, 3215-3226 (2000).

16. Leptin, M.twist and snail as positive and negative regulators during Drosophila mesoderm development. Genes Dev. 5, 1568-1576 (1991).

17. Cano, A. et al. The transcription factor Snail controls epithelial-mesenchymal transitions by repressing E-cadherin expression. Nat. Cell Biol. 2, 76-83 (2000).

18. Carver, E. A., Jiang, R., Lan, Y., Oram, K. F. \& Gridley, T. The mouse snail gene encodes a key regulator of the epithelial-mesenchymal transition. Mol. Cell Biol. 21, 8184-8188 (2001).

19. Peinado, H., Olmeda, D. \& Cano, A.Snail, ZEB and bHLH factors in tumour progression: an alliance against the epithelial phenotype? Nat. Rev. Cancer 7, 415-428 (2007)

20. Lamouille, S., Xu, J. \& Derynck, R. Molecular mechanisms of epithelialmesenchymal transition. Nat. Rev. Mol. Cell Biol. 15, 178-196 (2014).

21. Hatta, K. \& Takeichi, M. Expression of N-cadherin adhesion molecules associated with early morphogenetic events in chick development. Nature 320, 447-449 (1986)

22. Zohn, I. E. et al. p38 and a p38-interacting protein are critical for downregulation of E-cadherin during mouse gastrulation. Cell 125, 957-969 (2006)

23. Nieto, M. A., Bennett, M. F., Sargent, M. G. \& Wilkinson, D. G. Cloning and developmental expression of Sna, a murine homologue of the Drosophila snail gene. Development 116, 227-237 (1992).

24. Beddington, R. S. P. \& Robertson, E. J.Axis development and early asymmetry in mammals. Cell 96, 195-209 (1999).

25. Tam, P. P. L. et al. Sequential allocation and global pattern of movement of the definitive endoderm in the mouse embryo during gastrulation. Development 134, 251-260 (2006)

26. Tam, P. P. L. \& Beddington, R. S. P.Establishment and organization of germ layers in the gastrulating mouse embryo. Ciba Found. Symp. 165, 27-41 (2007). 
27. Rivera-Pérez, J. A. \& Hadjantonakis, A. K.The dynamics of morphogenesis in the early mouse embryo. Cold Spring Harb. Perspect. Biol. 7, a015867 (2015).

28. Viotti, M., Nowotschin, S. \& Hadjantonakis, A. K.SOX17 links gut endoderm morphogenesis and germ layer segregation. Nat. Cell Biol. 16, 1146-1156 (2014).

29. Burtscher, I., Barkey, W., Schwarzfischer, M., Theis, F. J. \& Lickert, H.The Sox17-mCherry fusion mouse line allows visualization of endoderm and vascular endothelial development. Genesis 50, 496-505 (2012).

30. Burtscher, I., Barkey, W. \& Lickert, H.Foxa2-Venus fusion reporter mouse line allows live-cell analysis of endoderm-derived organ formation. Genesis 51, 596-604 (2013).

31. Bergen, V., Lange, M., Peidli, S., Wolf, F. A. \& Theis, F. J.Generalizing RNA velocity to transient cell states through dynamical modeling. Nat. Biotechnol. 38, 1408-1414 (2020).

32. Lange, M. et al. CellRank for directed single-cell fate mapping. Preprint at bioRxiv https://doi.org/10.1101/2020.10.19.345983 (2020).

33. Wang, P. et al. A molecular signature for purified definitive endoderm guides differentiation and isolation of endoderm from mouse and human embryonic stem cells. Stem Cells Dev. 21, 2273-2287 (2012).

34. Cernilogar, F. M. et al. Pre-marked chromatin and transcription factor co-binding shape the pioneering activity of Foxa2. Nucleic Acids Res. 47, 9069-9086 (2019).

35. Takagi, T., Moribe, H., Kondoh, H. \& Higashi, Y.dEF1, a zinc finger and homeodomain transcription factor, is required for skeleton patterning in multiple lineages. Development 125, 21-31 (1998)

36. Liu, Y., El-Naggar, S., Darling, D. S., Higashi, Y. \& Dean, D. C.Zeb1 links epithelial-mesenchymal transition and cellular senescence. Development 135, 579-588 (2008).

37. Zhou, B. P. et al. Dual regulation of Snail by GSK-3 $\beta$-mediated phosphorylation in control of epithelial-mesenchymal transition. Nat. Cell Biol. 6, 931-940 (2004).

38. Artus, J. \& Hadjantonakis, A. K.Generation of chimeras by aggregation of embryonic stem cells with diploid or tetraploid mouse embryos. Methods Mol. Biol. 693, 37-56 (2011).

39. Muzumdar, M. D., Tasic, B., Miyamichi, K., Li, N. \& Luo, L.A global double-fluorescent Cre reporter mouse. Genesis 45, 593-605 (2007).

40. Haegel, H. et al. Lack of $\beta$-catenin affects mouse development at gastrulation. Development 121, 3529-3537 (1995).

41. Kemler, R. et al. Stabilization of $\beta$-catenin in the mouse zygote leads to premature epithelial-mesenchymal transition in the epiblast. Development 131, 5817-5824 (2004).

42. Ben-Haim, N. et al. The nodal precursor acting via activin receptors induces mesoderm by maintaining a source of its convertases and BMP4. Dev. Cell 11, 313-323 (2006).

43. Peinado, H., Quintanilla, M. \& Cano, A.Transforming growth factor $\beta-1$ induces Snail transcription factor in epithelial cell lines: mechanisms for epithelial mesenchymal transitions. J. Biol. Chem. 278, 21113-21123 (2003).

44. Yook, J. I. et al. A Wnt-Axin2-GSK3 $\beta$ cascade regulates Snaill activity in breast cancer cells. Nat. Cell Biol. 8, 1398-1406 (2006).

45. Jong, I. Y., Li, X. Y., Ota, I., Fearon, E. R. \& Weiss, S. J.Wnt-dependent regulation of the E-cadherin repressor snail. J. Biol. Chem. 280, 11740-11748 (2005)

46. Hovanes, K. et al. $\beta$-catenin-sensitive isoforms of lymphoid enhancer factor-1 are selectively expressed in colon cancer. Nat. Genet. $\mathbf{2 8}$, 53-57 (2001).
47. Filali, M., Cheng, N., Abbott, D., Leontiev, V. \& Engelhardt, J. F.Wnt$3 \mathrm{~A} / \beta$-catenin signaling induces transcription from the LEF-1 promoter. J. Biol. Chem. 277, 33398-33410 (2002).

48. Tamplin, O. J. et al. Microarray analysis of Foxa2 mutant mouse embryos reveals novel gene expression and inductive roles for the gastrula organizer and its derivatives. BMC Genomics 9, 511 (2008).

49. Cieply, B. et al. Suppression of the epithelial-mesenchymal transition by grainyhead-like-2. Cancer Res. 72, 2440-2453 (2012).

50. Chung, V. Y. et al. GRHL2-miR-200-ZEB1 maintains the epithelial status of ovarian cancer through transcriptional regulation and histone modification. Sci. Rep. 6, 19943 (2016).

51. Qi, X. K. et al. OVOL2 links stemness and metastasis via fine-tuning epithelial-mesenchymal transition in nasopharyngeal carcinoma. Theranostics 8, 2202-2216 (2018)

52 . Du, J. et al. $O$-fucosylation of thrombospondin type 1 repeats restricts epithelial to mesenchymal transition (EMT) and maintains epiblast pluripotency during mouse gastrulation. Dev. Biol. 346, 25-38 (2010).

53. Yeung, T. L. et al. ELF3 is a negative regulator of epithelial-mesenchymal transition in ovarian cancer cells. Oncotarget 8, 16951-16963 (2017).

54. Benz, B. A. et al. Genetic and biochemical evidence that gastrulation defects in Pofut2 mutants result from defects in ADAMTS9 secretion. Dev. Biol. 416, 111-122 (2016).

55. Yang, J. et al. Guidelines and definitions for research on epithelialmesenchymal transition. Nat. Rev. Mol. Cell Biol. 21, 341-352 (2020).

56. Townes, P. L. \& Holtfreter, J.Directed movements and selective adhesion of embryonic amphibian cells. J. Exp. Zool. 128, 53-120 (1955).

57. Zheng, X. et al. Epithelial-to-mesenchymal transition is dispensable for metastasis but induces chemoresistance in pancreatic cancer. Nature 527, 525-530 (2015).

58. Chen, Y. et al. Dual reporter genetic mouse models of pancreatic cancer identify an epithelial-to-mesenchymal transition-independent metastasis program. EMBO Mol. Med. 10, e9085 (2018).

59. Song, Y., Washington, M. K. \& Crawford, H. C.Loss of FOXA1/2 is essential for the epithelial-to-mesenchymal transition in pancreatic cancer. Cancer Res. 70, 2115-2125 (2010)

60. Wang, J. et al. FOXA2 suppresses the metastasis of hepatocellular carcinoma partially through matrix metalloproteinase-9 inhibition. Carcinogenesis 35, 2576-2583 (2014).

61. Tang, Y., Shu, G., Yuan, X., Jing, N. \& Song, J.FOXA2 functions as a suppressor of tumor metastasis by inhibition of epithelial-to-mesenchymal transition in human lung cancers. Cell Res. 21, 316-326 (2011).

Publisher's note Springer Nature remains neutral with regard to jurisdictional claims in published maps and institutional affiliations.

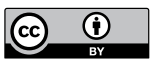

Open Access This article is licensed under a Creative Commons

Attribution 4.0 International License, which permits use, sharing, adaptation, distribution and reproduction in any medium or format, as long as you give appropriate credit to the original author(s) and the source, provide a link to the Creative Commons license, and indicate if changes were made. The images or other third party material in this article are included in the article's Creative Commons license, unless indicated otherwise in a credit line to the material. If material is not included in the article's Creative Commons license and your intended use is not permitted by statutory regulation or exceeds the permitted use, you will need to obtain permission directly from the copyright holder. To view a copy of this license, visit http://creativecommons. org/licenses/by/4.0/

(C) The Author(s) 2021, corrected publication 2021 


\section{Methods}

Mouse strains. $\mathrm{FVF}^{30}, \mathrm{SCF} \times \mathrm{FVF}^{29}, \mathrm{mT} / \mathrm{mG}^{39}$ and $\mathrm{CD}-1$ mice were kept and experiments were performed at the central facilities at the Helmholtz Zentrum München German Research Center for Environmental Health in accordance with German animal welfare legislation and acknowledged guidelines of the Society for Laboratory Animal Science and Federation of European Laboratory Animal Science Associations. Mice were kept under specific pathogen-free conditions in animal rooms with a light/dark cycle of $12 \mathrm{~h} / 12 \mathrm{~h}$, a temperature of $20-24^{\circ} \mathrm{C}$ and a humidity of $45-65 \%$. Mice received sterile filtered water and a standard diet for rodents ad libitum. For embryo generation, females at the age of $\geq 6$ weeks and males at the age of $\geq 8$ weeks were used.

Cell lines. The cell lines used during this study were as follows: $\mathrm{T}^{\mathrm{GFP} /+} ; \mathrm{Foxa} 2^{\operatorname{tagRFP} /+}$ (ref. ${ }^{62}$ ) G4 mESCs, FVF IDG3.2 mESCs $^{30}$, IDG3.2 mESCs $^{63}$, Snaill knockout IDG3.2 mESCs and Foxa2-H2B-Venus IDG3. $2 \mathrm{mESCs}^{34}$.

Gene targeting. The strategies for targeting $\mathrm{T}^{\mathrm{GFP} /+}$; Foxa $2^{\mathrm{tagRFP} /+}$ and Snail1 knockout constructs are outlined in Extended Data Figs. 3a and 5. To generate the $\mathrm{T}^{\mathrm{GFP} /+} ; \mathrm{Foxa}^{\mathrm{tagRFP} /+}$ dual-reporter mESC line, we generated a targeting vector where the open reading frame (ORF) of Foxa2 in exon 3 was fused to the ORF of the red fluorescent protein (RFP) tagRFP, followed by a phospho-glycerate kinase promoter-driven Neomycin (Neo) resistance gene cassette flanked by two loxP sites. The plasmid construct was introduced by electroporation into an available T-GFP knock-in mESCs line, in which the Brachyury expression was disrupted by insertion of a green fluorescent protein (GFP) mini gene ${ }^{62}$. Neo-resistant clones were analysed by Southern blot and PCR with the primers EP064, EP397, EP398 and EP1320 (Supplementary Table 2).

To generate Snaill knockout mESCs, we designed a targeting vector that replaced the Snaill ORF by an H2B-Venus-Intron-polyA Neo cassette ${ }^{64}$. Two guide RNAs (gRNAs) were designed to cut around the start codon of Snail 1 (gRNAs 101 and 129) and a further two gRNAs, used to cut around the stop codon of Snaill (gRNAs 4 and 7), were cloned into the pbs-U6 vector. mESCs were transfected with the Snail1 knockout targeting vector, four gRNAs and Cas9-D10A overexpression vector (pCAG-Cas9-D10A-bpA). At $48 \mathrm{~h}$ after transfection, cells were selected with G418 and picked clones were analysed by PCR genotyping (Extended Data Fig. 5 and Supplementary Table 2). The recombination borders of the targeting vector and wild-type sequence were analysed by sequencing of the knock-in-specific PCR product.

Aggregation chimeras. Aggregation chimeras were generated as described by Artus and Hadjantonakis ${ }^{38}$.

Immunofluorescence stainings of whole-mount embryos. Embryos (E6.5-7.5) were dissected in Dulbecco's phosphate-buffered saline (DPBS) and immediately fixed for $20 \mathrm{~min}$ with $2 \%$ paraformaldehyde (PFA) in DPBS at room temperature while shaking. The fixation was stopped by rinsing the embryos $2 \times$ with DPBS containing $0.1 \%$ Tween 20 (Merck; P9416) (DPBST). The embryos were permeabilized for $10 \mathrm{~min}(\leq \mathrm{E} 7.5)$ to $15 \mathrm{~min}(>\mathrm{E} 7.5)$ using $0.1 \mathrm{M}$ glycine (Merck; G8898) and 0.1\% Triton X-100 (Merck; 108643) in Milli-Q water, then rinsed $2 \times$ with DPBST. Unspecific antibody binding was prevented by incubating the embryos in blocking solution containing $0.1 \%$ Tween $20,10 \%$ heat-inactivated foetal calf serum (FCS), $0.1 \%$ bovine serum albumin (BSA) and 3\% donkey serum in DPBST for $\geq 1 \mathrm{~h}$ at room temperature while shaking. Subsequently, the primary antibodies diluted in blocking solution (for dilutions, see the antibody list in Supplementary Table 3 ) were added and the embryos were kept at $4{ }^{\circ} \mathrm{C}$ overnight on a shaker and for another $1-2 \mathrm{~h}$ at room temperature the following day. The embryos were rinsed $2 \times$ and washed $3 \times$ for $10 \mathrm{~min}$ with DPBST. Subsequently, the embryos were exposed to the secondary antibodies diluted in blocking solution for at least $3 \mathrm{~h}$ at room temperature on a shaker. The secondary antibodies were replaced by a 4',6-diamidino-2-phenylindole (DAPI)/DPBST solution $\left(2 \mu \mathrm{g} \mathrm{ml}^{-1}\right.$ DAPI in DPBST) and incubated for $20 \mathrm{~min}$ at room temperature. Afterwards, the embryos were rinsed twice and then washed $3 \times$ for $10 \mathrm{~min}$ with DPBST. The embryos were dehydrated in 15 and $30 \%$ glycerol in DPBS, each for $10 \mathrm{~min}$ at room temperature. The embryos were then embedded in antifade between two cover slips using a $100-\mu \mathrm{m}$ spacer, dried at room temperature and stored at $4{ }^{\circ} \mathrm{C}$ until imaging. A list of primary and secondary antibodies is shown in Supplementary Table 3.

Paraffin immunohistochemistry. Embryos were fixed in 4\% PFA overnight, embedded in HistoGel (Richard-Allan Scientific; HG-4000-144), dehydrated in alcohol gradients, embedded in wax blocks and sectioned at $6-\mu \mathrm{m}$ thickness. Slides were cleared with xylene, rehydrated and permeabilized in $1 \%$ sodium dodecyl sulfate (SDS) before performing antigen retrieval (Diva Decloaker; Biocare Medical).

Blocking was done with $10 \%$ normal donkey serum and incubation was performed with primary antibodies overnight at $4{ }^{\circ} \mathrm{C}$. Slides were then washed $3 \times$ with PBST and incubated with fluorophore-conjugated secondary antibodies for $1 \mathrm{~h}$ at room temperature in the dark. Nuclei were stained with DAPI (Life Technologies, 1:500-1:1,000). After washing, the slides were finally mounted with Vectashield (Vector Laboratories; H-1000-10) and imaged using a confocal microscope.

Immunofluorescence stainings of cryosections from embryos. Embryos were fixed for $30 \mathrm{~min}$ to $1 \mathrm{~h}$ in $2 \%$ PFA, washed twice in PBS and dehydrated in 5, 10, 15, 20 and $30 \%$ sucrose ( $1 \mathrm{~h}$ each) and 30\% sucrose/O.C.T. (Tissue-Tek; 4583) (1:1) overnight at $4{ }^{\circ} \mathrm{C}$. Embryos were sectioned at 12 - to $15-\mu \mathrm{m}$ thickness and stored at $-20^{\circ} \mathrm{C}$ until usage. The sectioned embryos were permeabilized and blocked (see above). Primary and secondary antibody staining was performed as described above. Finally, the slides were mounted with Vectashield and kept for $24 \mathrm{~h}$ at room temperature to dry. Supplementary Table 3 lists the antibodies and dilutions used.

Immunofluorescence stainings of cells. Differentiated cells were washed with PBS and fixed for $15 \mathrm{~min}$ with $4 \%$ PFA. The cells were immunostained as described in the previous section. The cells were kept in PBS for immediate imaging. Detailed information about the primary and secondary antibodies used is provided in Supplementary Table 3 .

Immunofluorescence stainings for flow cytometry analysis. Differentiated mESCs were dissociated using Accutase (Merck; A6964) or $0.05 \%$ trypsin (Life Technologies; 25300054) and fixed in 4\% PFA for $10 \mathrm{~min}$. The cells were permeabilized for $15 \mathrm{~min}$ and blocked for $1 \mathrm{~h}$ at room temperature (see above). Next, primary antibodies were diluted in blocking solution and incubated for $3-4 \mathrm{~h}$ at room temperature or overnight at $4{ }^{\circ} \mathrm{C}$. Cells were washed $3 \times$ with PBS for $10 \mathrm{~min}$ each and secondary antibody solution was added for $1-2 \mathrm{~h}$ at room temperature. After another washing of $3 \times$ with PBS, samples were analysed by BD FACSAria III and FlowJo version 10.2. The gates were determined by secondary antibody controls. A list of all primary, secondary and conjugated antibodies is provided in Supplementary Table 3.

\section{Fluorescence-activated cell sorting (FACS) for scRNA-seq and western} blotting. Embryos were isolated and extraembryonic compartments were removed mechanically. Embryonic compartments were washed with cold PBS. For single-cell suspension, FVF embryos and differentiated Foxa $2^{\text {Venus } /+}$ mESCs were incubated with TrypLE (Life Technologies; 12605) for $10 \mathrm{~min}$ at $37^{\circ} \mathrm{C}$. Cells were dissociated by pipetting up and down. To exclude dead cells, samples were incubated for 5-10 min with either 7-aminoactinomycin D or DAPI on ice in the dark. The samples were then washed twice, resuspended in FACS buffer (PBS, $2 \%$ FCS and $2 \mathrm{mM}$ ethylenediaminetetraacetic acid) and loaded for flow sorting. The gating strategy was as follows: main population $>$ single cells $>$ living cells (7-aminoactinomycin D/DAPI negative) $>$ FVF $^{\text {neg }}$, FVF ${ }^{\text {low }}$ and FVF high cells. For scRNA-seq analysis, the cells were collected in RNA-seq buffer (PBS and 1\% FCS). For western blotting analysis, the cells were collected in PBS and spun down and the pellet was snap frozen until it was used.

Western blotting. For western blotting of FACS-sorted cells, the cell pellet was dissociated by RIPA buffer ( $75 \mathrm{mM} \mathrm{NaCl}, 6.37 \mathrm{mM}$ sodium deoxycholate, $0.005 \%$ NP-40, $0.05 \%$ SDS and $25 \mathrm{mM}$ Tris ( $\mathrm{pH} 8)$ ) supplemented with phosphatase inhibitor cocktail. The cell lysates were resolved by SDS-polyacrylamide gel electrophoresis, then transferred to a polyvinylidene fluoride membrane (Bio-Rad) and blocked in $5 \%$ BSA-TBST (TBS $+0.1 \%$ Tween 20 ) for $1 \mathrm{~h}$. The primary antibodies were incubated in $5 \%$ BSA-TBST overnight at $4{ }^{\circ} \mathrm{C}$ on a shaker. The following day, the membranes were washed at least three times for $15 \mathrm{~min}$ in TBST on a shaker. The secondary horseradish peroxidase-conjugated antibodies were incubated with shaking for $1-2 \mathrm{~h}$ at room temperature. After washing the membranes with TBST three times for $15 \mathrm{~min}$ each, the bands were visualized by adding Pierce ECL Western Blotting Substrate (Thermo Fisher Scientific). The bands were quantified with ImageJ version 1.53c. Supplementary Table 3 lists the primary and secondary antibodies and their dilutions.

Image analysis. Images from immunostained embryos were acquired with Leica SP5 and Zeiss LSM 880 Airyscan confocal microscopes. Images taken with the Leica confocal microscope were analysed using Leica LAS AF Lite 4.0 and images taken with the Zeiss confocal microscope were processed using Zeiss Zen 2.3 lite Blue software.

Quantifications. Western blots were quantified with ImageJ version 1.53c by determining the pixel density of the protein of interest. After background subtraction, the ratio was determined by protein of interest/loading control.

For all of the quantifications of different cell populations, an entire confocal $z$ stack of an embryo was analysed using ImageJ version $1.53 \mathrm{c}$. For every staining, three individual embryos were processed in the same way. In brief, for Fig. $3 \mathrm{~h}$, Sox $17^{+} \mathrm{mT}^{-}$(definitive endoderm) cells and Sox $17^{+} \mathrm{mT}^{+}$(visceral endoderm) cells at the surface of the Snaill variant and wild-type embryos were counted. For Fig. 4h, Venus ${ }^{\text {high }}$ and either Snail ${ }^{+}$or Snail1 ${ }^{-}$cells were counted throughout the heterozygous and homozygous Foxa2 variant embryos. For Fig. 6g, Venus ${ }^{\text {high }}$ or Foxa $2^{\text {high }}$ cells co-expressing Snail 1 and/or Lef $1^{\text {low/high }}$ were counted throughout the entire wild-type or Foxa2 variant embryos, excluding the visceral endoderm/ definitive endoderm at the surface of the embryo. For Extended Data Fig. 1g, 
Foxa2, T and Snail $1^{\text {low/mid/high }}$-expressing cells in mesoderm, AME and Foxa $2^{\text {high }}$ transitory progenitors (based on anatomical position and marker expression) were analysed throughout a complete wild-type embryo, excluding the visceral endoderm/definitive endoderm at the surface of the embryo. For Extended Data Fig. 1i, $\mathrm{FVF}^{+}$epiblast progenitors, FVF, Foxa $2^{\text {high }}$ transitory progenitors and Foxa $2^{\text {high }}$ visceral endoderm/definitive endoderm (based on anatomical location and marker expression) were quantified for E-cadherin and $\mathrm{N}$-cadherin expression in FVF embryos. In Extended Data Fig. 1h, Foxa $2^{\text {high }}$ transitory progenitors, Foxa $2^{\text {high }}$ transitory progenitors/visceral endoderm/definitive endoderm and AME (based on anatomical position and marker expression) were analysed for Foxa2, Sox17 and Snaill expression in wild-type embryos. In Extended Data Fig. $7 \mathrm{c}$, the intensity of E-cadherin was measured in a rectangular area at three different positions within the anterior or posterior epiblast from one $z$ plane of FVF embryos. The intensity of Claudin7 was measured in a rectangular area at three different positions within the anterior or posterior epiblast of wild-type embryos.

Cell culture and differentiation. Mouse ESCs were cultured on mitomycin C-treated mouse embryonic fibroblasts (feeders) in Dulbecco's modified Eagle's medium (Life Technologies; 11965092) supplemented with 15\% FCS (PAN-Biotech; P30-2602), 0.1 mM $\beta$-mercaptoethanol (Life Technologies; 31350 10), $2 \mathrm{mM}$ L-glutamine (Life Technologies; 25030081), $1 \times$ non-essential amino acids (Sigma-Aldrich; M7145), 2 mM HEPES (Life Technologies; 15630-056) and $1,000 \mathrm{U} \mathrm{ml}^{-1}$ leukaemia inhibitory factor (Sigma-Aldrich; ESG1107). Every $2-3 \mathrm{~d}$, cells were passaged by treatment with $0.05 \%$ trypsin (Life Technologies; 25300054) on new feeders and the medium was changed every day. For differentiations of the Snaill knockout mESCs, $\mathrm{T}^{\mathrm{GFP} /+}$; Foxa $2^{\mathrm{tagRFP} /+}$ mESCs and IDG3. $2 \mathrm{mESCs}, 1 \times 10^{5}$ cells per $1 \mathrm{~cm}^{2}$ were seeded in chemically defined medium, as published recently ${ }^{65}$. After $24 \mathrm{~h}$, endoderm was induced by the addition of $2.5 \mu \mathrm{M}$ CHIR99021 (Miltenyi Biotec; 130-103-926) for $1 \mathrm{~d}$ and $25 \mathrm{ng} \mathrm{ml}^{-1}$ activin A (Peprotech; 120-14-300) for all $3 \mathrm{~d}$. For the inhibitor experiments, endoderm was induced as described before, and on days 2 and 3 either $400 \mathrm{ng} \mathrm{ml}^{-1}$ DKK1 (Peprotech; 120-30-50) or 1.25 $\mu$ M IWP2 (Tocris; 3533-10) was supplemented. Differentiations of the FVF mESCs and Foxa2 $2^{\text {Venus/Venus }}$ knockout mESCs were performed as described before ${ }^{34}$. The differentiation of $\mathrm{T}^{\mathrm{GFP} /+}$; Foxa $2^{\text {tagRFP/+ }}$ mESCs followed by microarray analysis was carried out based on a previously published protocol $^{34}$. Briefly, before differentiation, Wnt3a feeders were seeded at a density of $\sim 5 \times 10^{4}$ cells per 24 wells. ESCs were passaged onto gelatine-coated plates for 30 min twice to remove remaining feeders from the maintenance cultures. Next, ESCs were transferred to a Wnt3a-expressing feeder plate with a seeding density of $2 \times 10^{5}$ cells per 24 wells. Endoderm differentiation medium, consisting of $500 \mathrm{ml}$ advanced Dulbecco's modified Eagle's medium/F-12 (Life Technologies; 12634-10), $500 \mathrm{ml}$ advanced RPMI 1640 (Life Technologies; 12633-012), 22 ml GlutaMAX (Life Technologies; 12860-01), $200 \mu$ l AlbuMAX $100 \mathrm{mg} \mathrm{ml}^{-1}$ (Life Technologies; 11021-029), $22 \mathrm{ml}$ HEPES $1 \mathrm{M}, 70 \mu \mathrm{l}$ cytidine $150 \mathrm{mg} \mathrm{ml}^{-1}$ (Sigma-Aldrich; C4654), $0.9 \mathrm{ml} \beta$-mercaptoethanol $50 \mathrm{mM}, 12 \mathrm{ml}$ penicillin/streptomycin $10,000 \mathrm{U} \mathrm{ml}^{-1}$ (Life Technologies; 10378016) and $1 \mathrm{ml}$ Insulin-Transferrin-Selenium-Ethanolamine (Life Technologies; 51500-056) supplemented with $12 \mathrm{ng} \mathrm{ml}^{-1}$ activin A, was used to induce endoderm differentiation. For mesoderm differentiation, $3 \mathrm{ng} \mathrm{ml}^{-1}$ activin $\mathrm{A}$ and $7.5 \mathrm{ng} \mathrm{ml}^{-1} \mathrm{Bmp} 4$ were added to the endoderm differentiation medium. The medium was changed every day.

Affymetrix microarray analysis. RNA was extracted using an miRNeasy Mini Kit (Qiagen; 217004) and total RNA (150 ng) amplified using the Ambion WT Expression Kit and the WT Terminal Labeling Kit (Affymetrix). Amplified complementary DNA was hybridized on Affymetrix Mouse Gene ST 1.0 arrays. Staining and scanning (Fluidics Script FS450_0007) were done according to the Affymetrix expression protocol. An expression console (version 1.2; Affymetrix) was used for quality control. CEL files were imported into R (3.6.3) and robust multichip average normalized using the oligo (1.48.0) package. Data were then pre-filtered and 32,000 probesets with the highest mean expression across all samples were kept for further analysis. Genewise testing for differential expression was performed using limma (3.40.6) and genes with an absolute $\log _{2}$ [fold change] of $>1$ and an adjusted $P<0.05$ were defined as differentially expressed. All microarray data are available from the (NCBI) Gene Expression Omnibus (GEO) under accession GSE148226.

RNA-seq data analysis. RNA-seq data from Foxa2 knockout definitive endoderm (processed ${ }^{*}$ rsem.genes.results.txt.gz files) were downloaded from the National Center for Biotechnology Information (NCBI) GEO (GSE116262) and analysed in $\mathrm{R}$ (3.6.3). RSEM output files were imported into $\mathrm{R}$ using the tximport (1.12.3) package and batch correction was performed using the RUVr function $(k=1)$ from the RUVseq (1.18.0) package. Counts were then prepared for DESeq2 (1.20.0) and genes with a minimum mean count below 5 were filtered out. DESeq 2 was run with default parameters and log[fold change] shrinkage was performed using apeglm (1.6.0) with the options svalue $=$ TRUE, lfcThreshold $=\log _{2}[1.5]$. Differentially expressed genes were defined by an $s$ value ${ }^{66}$ of $<0.05$.

ChIP-seq data analysis. Foxa2 ChIP-seq raw data (FASTQ files) were downloaded from the NCBI GEO (GSE116262). Trimmomatic (0.39) was used to trim low-quality bases and adapter contaminations, and Bowtie 2 (2.3.5.1) with the --very-sensitive option was used to map reads to the $\mathrm{mm} 10$ genome. Peaks were called using GEM (3.4), with the options --k_min 10 --k_max 13, and filtered using $q$ value cut-offs of $10^{-4}$. Binding sites were then mapped to putative target genes by assigning peaks within 20 kilobases of a transcription start site or within a gene body to the respective gene. Genes with Foxa2 binding sites that were regulated in the Foxa2 knockout RNA-seq data were considered to be regulated by Foxa2. The list of genes is provided in Supplementary Table 1.

Single-cell RNA-seq. Single-cell libraries were generated using the Chromium Single Cell 3' library and Gel Bead Kit version 2 (10X Genomics; 120237) according to the manufacturer's instructions. Libraries were sequenced on the HiSeq 4000 (Illumina) with 150-base pair paired-end sequencing of read 2.

Computational analysis of scRNA-seq data. Pre-processing of droplet-based scRNA-seq data. Demultiplexing and alignment to the mm10 mouse genome, identification of unique molecular identifiers (UMIs) and barcode filtering were performed using the Cell Ranger toolkit (version 2.0.0) provided by 10X Genomics. In addition, the velocyto pipeline ${ }^{67}$, which generated a loompy file, was used to determine unspliced and spliced genes. We considered only cells with at least 1,000 expressed genes, where a gene was counted as expressed if we found at least one UMI mapped to it. We further filtered cells with a total UMI count of $>125,000$ or a fraction of counts from mitochondrial genes of $>8 \%$, indicative of stressed or dying cells. We did not apply a minimum library size filter because almost all cells had a total UMI count above 5,000. Cells from all samples were divided by library size, with a target sum of 50,000 UMIs (counts per million normalization; pp.normalize_per_cell in scanpy version 1.4.5.2.dev6+gfa408dc7 in Python 3.7 ${ }^{68}$, and subsequently $\log +1$ scaled. Then, 3,000 highly variable genes were computed as follows: genes were binned in 20 groups by mean expression and a normalized dispersion was obtained by scaling with the mean and s.d. of the dispersions for genes falling into a given bin for mean expression of genes (pp.highly_variable_ genes in scanpy version 1.4.5.2.dev6+gfa408dc7 in Python 3.7 with the flavour cell_ranger to compute normalized dispersions), thereby accounting for differences across batches. Batch-effect correction was done with Scanorama version 1.4 (ref. ${ }^{69}$ ) using the corrected feature space. Spliced and unspliced UMI matrices were filtered with a minimum of 20 UMIs each. Then, the data were counts per million normalized and $\log +1$ scaled. Furthermore, the dataset was restricted to the same highly variable genes as the count matrix.

Dimension reduction. We performed our analyses with scanpy version 1.4.5.2.dev6+gfa408dc7 in Python 3.7. First, we computed a principal component analysis (PCA) space with $n=50$ components and a $k$-nearest neighbour graph on the PCA space with $k=30$ (tl.pca and pp.neighbors). PCA and UMAP embeddings was computed on the batch-corrected and normalized data with 3,000 highly variable genes. We then used $\mathrm{UMAP}^{70}$ to represent the data in the two-dimensional embedding (tl.umap).

Clustering and cell-type annotation. The data matrix was clustered with the Louvain algorithm (tl.louvain with resolution 2.0 ( $^{71}$ and found 27 clusters. All clusters were then inspected for potential substructure and five clusters were further resolved with a low resolution of 0.2 . Here, we annotated and merged clusters again according to marker gene expression, resulting in 11 cell stages. Marker gene expression was visualized in a dotplot using scanpy version 1.6.0. Furthermore, we classified the cells according to the cell sorting into FVF ${ }^{\text {neg }}, \mathrm{FVF}^{\text {low }}$ and FVF ${ }^{\text {high }}$ cells, respectively, determined the amount of Foxa 2 mRNA in each cell and classified Foxa2 mRNA-negative and -positive cells based on non-batch-corrected values, where every cell counted as positive with at least one UMI of the Foxa2 gene detected. In FVF ${ }^{\text {neg }}$ samples, the number of Foxa2 mRNA-positive cells was 45 out of 4,173 cells and we considered both categories as FVF ${ }^{\text {neg }}$.

Differential expression analysis. To study differential expression, we used the default scanpy test function (tl.rank_genes_groups with default method t-test_overestim _ var and Benjamini-Hochberg correction for multiple testing) to determine the pairwise differences of several populations (that is, epiblast and posterior epiblast, Foxa $2 \mathrm{mRNA}^{+}$and Foxa $2 \mathrm{mRNA}^{-}$posterior epiblast, posterior epiblast and transitory progenitors). We then visualized the top 100 up- and downregulated differentially expressed genes, respectively.

Identifying cell differentiation trajectories. To derive cell trajectories, we computed a pseudotemporal ordering using diffusion pseudotime (tl.dpt in scanpy) as a basis for subsequent visualization of gene trajectories towards the endoderm and mesoderm lineage. Next, we used the dynamical model of the scvelo package version 0.2.1 (ref. ${ }^{31}$ ) in scanpy version 1.5.1 to determine gene dynamics and potential cell fate decisions towards the endoderm and mesoderm lineage (tl.recover_dynamics and tl.velocity from the scVelo package). To further characterize the cell fate decision process and to leverage the sorting information of the FVF reporter, we used the CellRank package version 1.0.0-rc.0 (ref. ${ }^{32}$ ). Specifically, we recomputed the dynamical RNA velocity model excluding extraembryonic tissues and used it in CellRank, which creates a vector field from 
the data and uses a random walk model to determine initial, intermediate and terminal states in the data (metastable states). We identified six metastable states in the epiblasts (initial), posterior epiblast (intermediate), AME, definitive endoderm, lateral plate mesoderm and nascent endothelium (all terminal). Using the random walk model, we determined a fate probability (that is, the probability of each cell ending up in one of the metastable states). The fate probability of each cell was displayed in a uniform manifold approximation and projection (UMAP) plot, where the colour of the cell was determined by the cluster with the highest fate probability.

To determine the topology of the data and cluster-wise relationships of the cells, we used partition-based graph abstraction (PAGA $)^{72}$ to quantify the connections between the clusters (tl.paga in scanpy version 1.5.1). We display all connections with a scaled connectivity of at least 0.05 (threshold parameter in pl.paga in scanpy) and visualized the fate probability of each cell population as a pie chart (pl.cluster_fates with the mode paga-pie in CellRank). We further determined the differences in fate probability in the posterior epiblast FVF-sorted Foxa 2 mRNA-classified cells and displayed the fate probability per group as a bar chart (pl.cluster_fates with the mode bar in CellRank).

We further used CellRank's compute_lineage_drivers function to determine lineage drivers for each metastable state as the correlation of the gene expression with the respective lineage. Then, we highlighted the correlation with lateral plate mesoderm and definitive endoderm in a scatter plot.

Statistics and reproducibility. In general, all of the experiments were performed, if possible, with at least three independent biological samples. Sample sizes are provided in the figure captions. Fewer than three independent experiments were used for the FVF embryo sorting and western blot analysis due to the high quantity of embryos required for this experiment. However, a total number of 158 embryos in two independent experiments was used and considered as sufficient. No statistical method was used to predetermine sample size. Data were excluded when immunohistochemical stainings were insufficient, mESC differentiations failed $\left(<2 \%\right.$ Foxa $2^{+}$cells), embryos were ruptured or embryos were at the wrong stage. For the scRNA-seq analysis, one of the FACS sorted FVF ${ }^{\text {neg }}$ samples (FVF_neg_3) was excluded from the analysis due to low sequencing depth. The experiments were randomized if possible. The investigators were not blinded to allocation during the experiments and outcome assessment. All replications were successful. The data were analysed using GraphPad Prism software 8 (GraphPad Software).

Reporting summary. Further information on experimental design is available in the Life Sciences Reporting Summary linked to this article.

\section{Data availability}

The sequencing data that support the findings of this study have been deposited in the Gene Expression Omnibus (GEO) under the accession codes GSE148226 and GSE162534. Previously published sequencing data that were re-analysed here are available under accession codes GSE116262 (samples GSM3223321, GSM3223325, GSM3223326, GSM3223342-GSM3223345, SM3597790 and GSM3597791) Source data are provided with this paper. All other data supporting the findings of this study are available from the corresponding author upon reasonable request.

\section{Code availability}

The analyses of scRNA-seq data are available at https://github.com/theislab/ gastrulation_analysis.

\section{References}

62. Fehling, H. J. et al. Tracking mesoderm induction and its specification to the hemangioblast during embryonic stem cell differentiation. Development 130 4217-4227 (2003).

63. Hitz, C., Wurst, W. \& Kühn, R.Conditional brain-specific knockdown of MAPK using Cre/loxP regulated RNA interference. Nucleic Acids Res. 35, e90 (2007).
64. Gegg, M. et al. Flattop regulates basal body docking and positioning in mono- and multiciliated cells. eLife 3, e03842 (2014).

65. Mfopou, J. K. et al. Efficient definitive endoderm induction from mouse embryonic stem cell adherent cultures: a rapid screening model for differentiation studies. Stem Cell Res. 12, 166-177 (2014).

66. Stephens, M.False discovery rates: a new deal. Biostatistics 18, 275-294 (2017).

67. La Manno, G. et al. RNA velocity of single cells. Nature 560, 494-498 (2018).

68. Wolf, F. A., Angerer, P. \& Theis, F. J.SCANPY: large-scale single-cell gene expression data analysis. Genome Biol. 19, 15 (2018).

69. Hie, B., Bryson, B. \& Berger, B.Efficient integration of heterogeneous single-cell transcriptomes using Scanorama. Nat. Biotechnol. 37, 685-691 (2019).

70. McInnes, L., Healy, J., Saul, N. \& Großberger, L.UMAP: uniform manifold approximation and projection. J. Open Source Softw. 3, 861 (2018).

71. Blondel, V. D., Guillaume, J. L., Lambiotte, R. \& Lefebvre, E. Fast unfolding of communities in large networks. J. Stat. Mech. 2008, P10008 (2008).

72. Wolf, F. A. et al. PAGA: graph abstraction reconciles clustering with trajectory inference through a topology preserving map of single cells. Genome Biol. 20, 59 (2019).

\section{Acknowledgements}

This work was supported by the Helmholtz-Gemeinschaft (Helmholtz Portfolio Theme 'Metabolic Dysfunction and Common Disease'), Deutsches Zentrum für Diabetesforschung (DZD) and Helmholtz Alliance 'Aging and Metabolic Programming' (AMPro) (J.B., M.I. and H.L.). K.S. is supported by the HELENA Graduate School and Technische Universität München. This research was funded in part by Wellcome Trust grant 108413/A/15/D (F.J.T. and M.B.). For the purpose of open access, F.J.T. has applied a CC BY public copyright licence to any author accepted manuscript version arising from this submission. F.J.T. acknowledges support from the Helmholtz Association's Initiative and Networking Fund through Helmholtz AI (grant number ZT-I-PF-5-01; F.J.T. and M.B.). Work in the laboratory of G.S. was funded by Deutsche Forschungsgemeinschaft grants SFB1064-A11 and SFB1321-P13 (G.S. and F.M.C.). We thank M. Bakhti for reading and discussing the manuscript; L. Appel, A. Ziegler, B. Vogel, J. Beckenbauer, W. Barkey and A. Bettenbrock for excellent technical assistance; and C. Hübner Freitag from the Helmholtz Core Facility Pathology \& Tissue Analytics for excellent assistance with histology.

\section{Author contributions}

K.S. and H.L. wrote the manuscript. The experiments were designed, analysed and interpreted by K.S., S.S, I.B. and H.L.. K.S., S.S. and I.B. performed the experiments. M.B and F.J.T. analysed the scRNA-seq data. D.Y. helped to generate the $\mathrm{T}^{\mathrm{GFP} /+}$; Foxa2 $2^{\operatorname{tagRFP} /+}$ mESC line and differentiated and sorted the $\mathrm{T}^{\mathrm{GFP} /+}$; Foxa $2^{\mathrm{tagRFP} /+}$ mESCs for further microarray analysis performed by J.B. and M.I. M.S. provided bioinformatics assistance and analysed the results. A.B. performed the FACS of gastrula embryos and sample processing for scRNA-seq analysis. A. performed stainings on paraffin sections. G.S. and F.M.C. performed the RNA-seq and ChIP-seq experiments.

\section{Competing interests}

F.J.T. reports receiving consulting fees from and having an ownership interest in Cellarity.

\section{Additional information}

Extended data is available for this paper at https://doi.org/10.1038/s41556-021-00694-x.

Supplementary information The online version contains supplementary material available at https://doi.org/10.1038/s41556-021-00694-x.

Correspondence and requests for materials should be addressed to H.L.

Peer review information Nature Cell Biology thanks the anonymous reviewers for their contribution to the peer review of this work.

Reprints and permissions information is available at www.nature.com/reprints. 


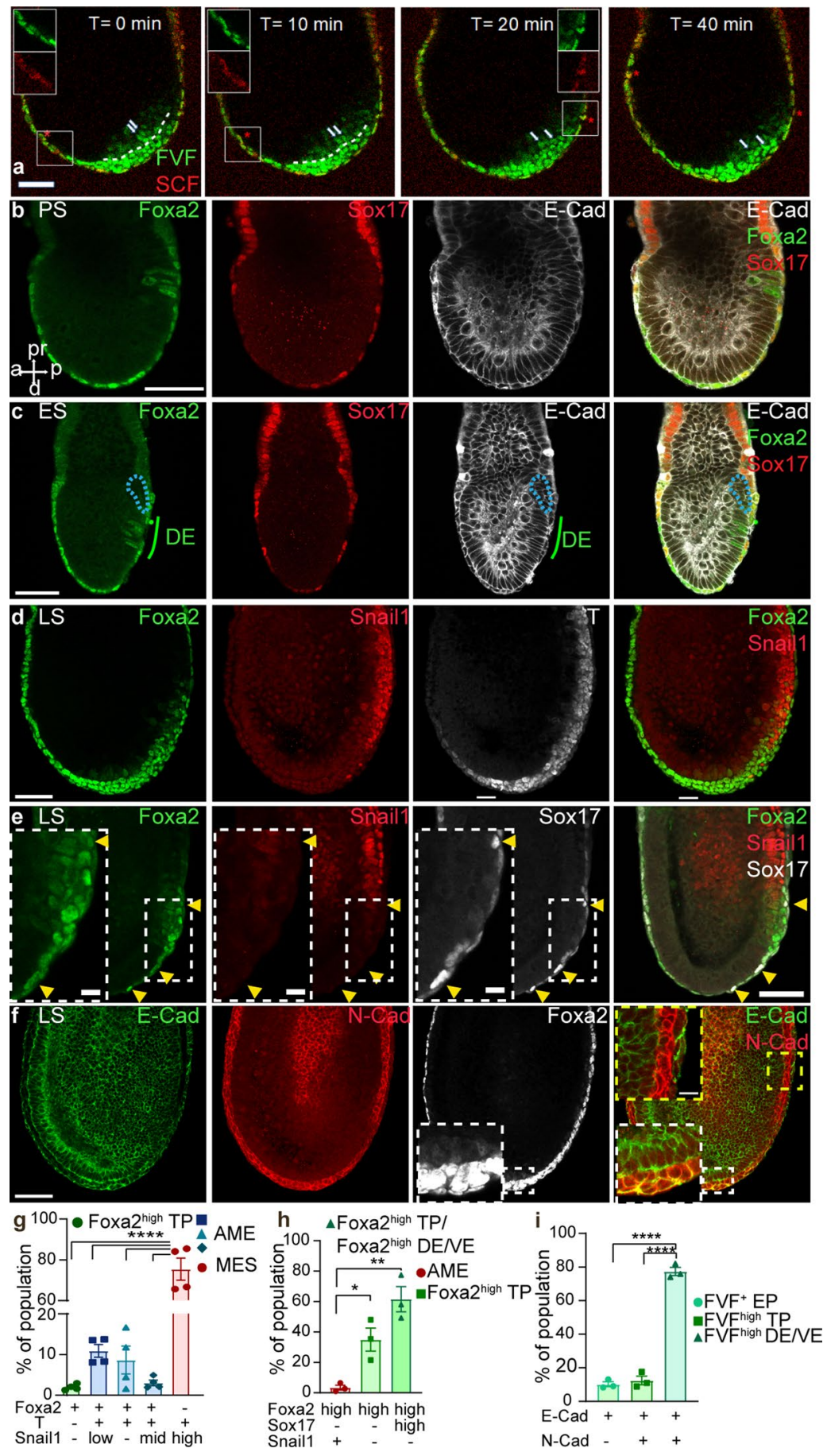

Extended Data Fig. 1 | The endoderm is formed independent of a full EMT-MET cycle. a, Still images extracted from live cell imaging of a LS stage FVF/ SCF embryo (representative of 4 embryos). The images show a single z-plane of the merge of the FVF channel in green and SCF channel in red. White dashed line shows the border of the epiblast, separating FVFlow and FVFigh expressing cells. The yellow arrows show a single FVF cell that increases fluorescent intensity; red asterisks: DE progenitor upregulating Sox17 and intercalating into the DE layer. b, Immunohistochemistry of E6.25 (pre-streak) (2 embryos) and E6.5 (early-streak stage) (8 embryos) FVF/SCF embryos stained for Venus (Foxa2), E-cadherin and RFP (Sox17) showing Foxa2 expressing cells appearing before PS is present and (c) after PS induction (8 embryos) Foxa2 expressing cells are distal to the PS (blue dashed line indicates PS). d,e, Immunohistochemistry of LS stage FVF embryo (7 embryos) stained for Venus (Foxa2), Snail1 and T (d), LS stage wildtype embryo (3 embryos) stained for Foxa2, Snail1 and Sox17. Note, Foxa2 ${ }^{\text {high }} /$ Sox 17+ TP does not express Snail1 (yellow arrowhead) (e). f, LS stage FVF embryo (3 embryos) immunostained for Venus (Foxa2), N-cadherin and E-cadherin. $\mathbf{g}$, Quantification of Foxa2, T and Snail1 positive cells in MES, AME, Foxa2high TP of MS stage embryos (Ordinary one-way ANOVA with Tukey's multiple comparison test, $n=4$ embryos). h, Quantification of Foxa2, Sox17 and Snail1 expression in AME, Foxa $2^{\text {high }}$ TP/Foxa2 ${ }^{\text {high }}$ DE/VE and Foxa $2^{\text {high }}$ TP in MS stage embryos (Ordinary one-way ANOVA with Tukey's multiple comparison test, $n=3$ embryos). $\mathbf{i}$, Quantification of E-cadherin and N-cadherin expression in FVF+, FVFhigh TP, FVFhigh DE/VE of MS stage embryos (Ordinary one-way ANOVA with Tukey's multiple comparison test, $\mathrm{n}=3$ embryos). All samples are derived from biologically independent experiments. Data are presented as mean values \pm SEM.; ${ }^{\star \star \star \star} P\left(0.0001,{ }^{\star \star} \mathrm{P}<0.0019,{ }^{\star} \mathrm{P}<0.0337\right.$. Statistically non-significant results are not indicated in the figure. All shown confocal images are single planes of a z-stack unless otherwise stated. Scale bar: $50 \mu \mathrm{m}$, insets $10 \mu \mathrm{m}$. 
b

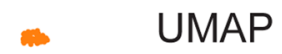

a

Foxa2-Venus Fusion embryos

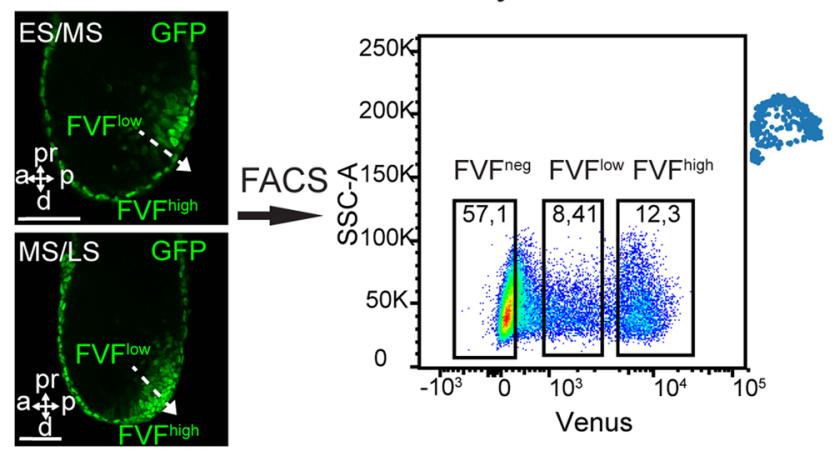

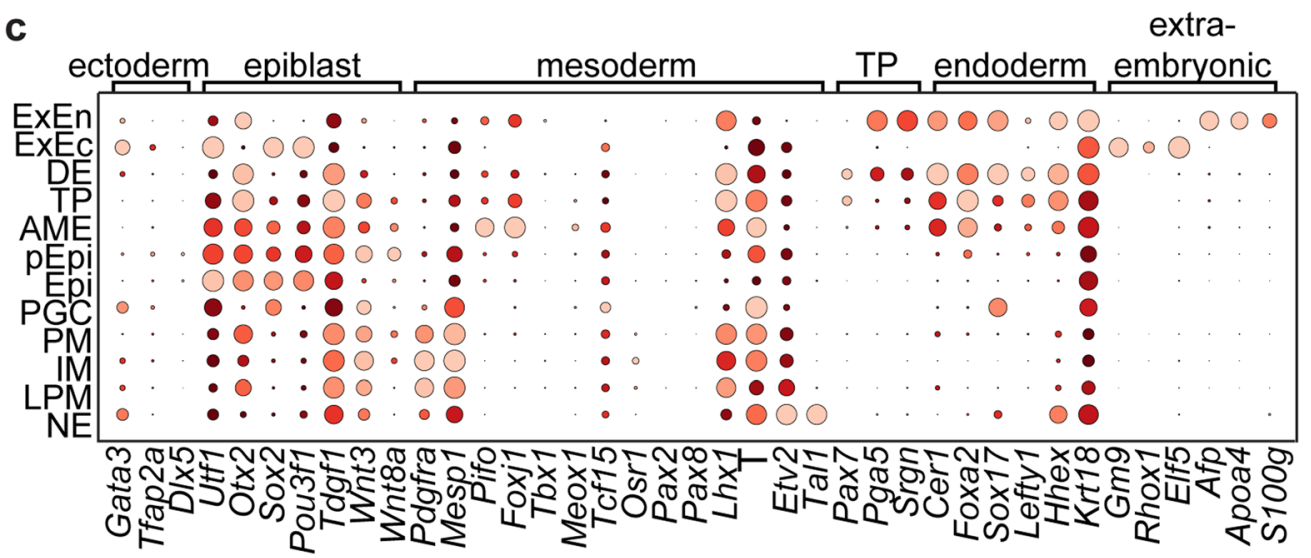

Fraction of cells Mean expression in group in group (\%) 20406080100

e
FVF'igh_

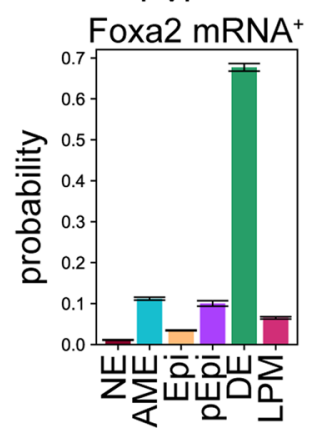

FVF'igh_

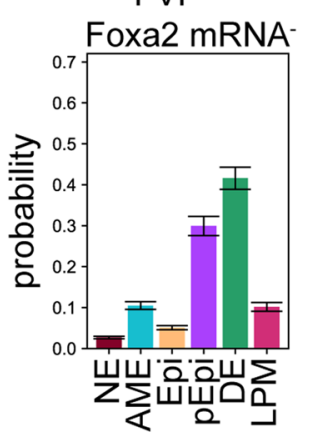

FVFlow_

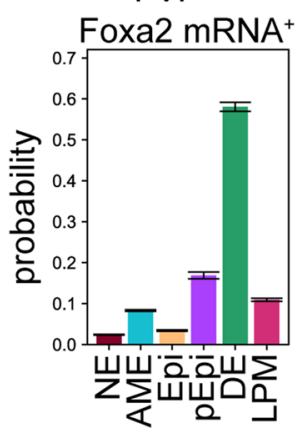

FVFlow_

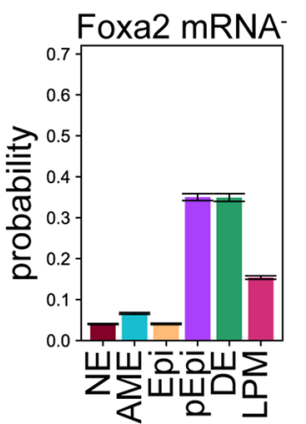

- extraembryonic endoderm (ExEn) - extraembryonic ectoderm (ExEc) - definitive endoderm (DE)

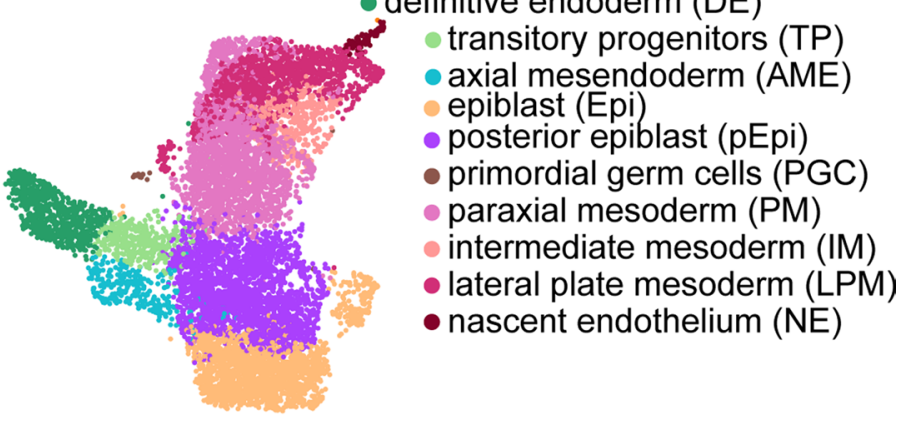

d scVelo UMAP

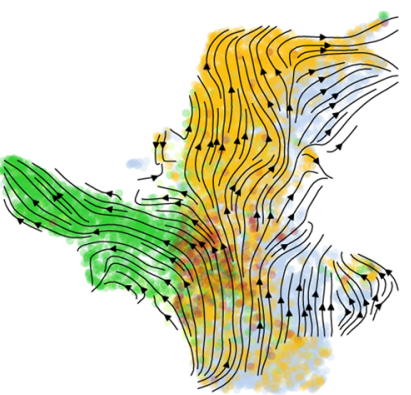

- FVF'igh_Foxa2 mRNA ${ }^{+}$ FVFigh-Foxa2 mRNA- FVFlow_Foxa2 mRNA ${ }^{+}$ -FVFlow_Foxa2 mRNAFVFreg

Extended Data Fig. 2 | Mapping and single cell transcriptional profiling of gastrulation. a, Scheme for FACS sorting of FVF embryos for scRNA-seq analysis (2 FVFneg, 3 FVFlow and 3 FVFhigh samples were used in the scRNAseq analysis). b, UMAP plot colored by cell population. $\mathbf{c}$, Dotplot of tissue-specific marker genes (top brackets). Colors indicate expression level, dot size indicates fraction of cells expressing the gene. d, UMAP plot with RNA velocity arrows, colored by both FVF-sorting and presence/absence of Foxa2 mRNA. RNA velocity shows gene dynamics derived from abundance of unspliced and spliced mRNA molecules for each gene. e, Barplots of the fate probability for the FVF-sorted and Foxa2 mRNA positive or negative subpopulations of the $p$ Epi (cell numbers from left to right, $n=308 ; n=52 ; n=287 ; n=428 ; n=1140$ ). Bar height denotes mean fate probability per state and upper/lower whiskers indicate SEM. The higher a bar, the more likely is the indicated fate for this group of cells. All shown confocal images are single planes of a z-stack unless otherwise stated. Scale bar: $50 \mu \mathrm{m}$. 
a wild type allele:

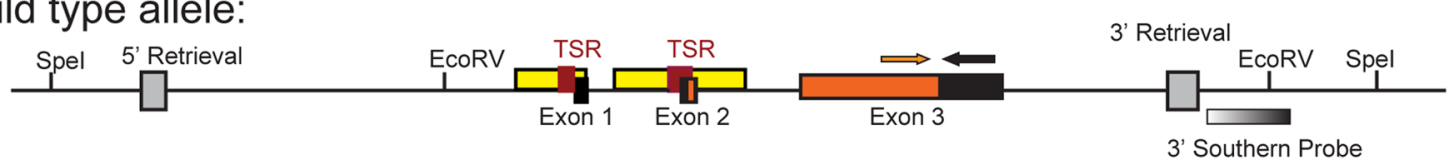

targeting vector:

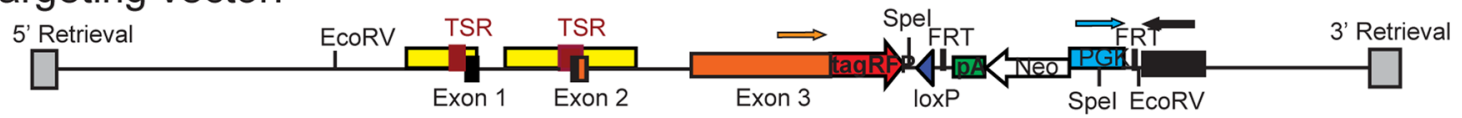

targeted Foxa2-tagRFP-Neo allele:
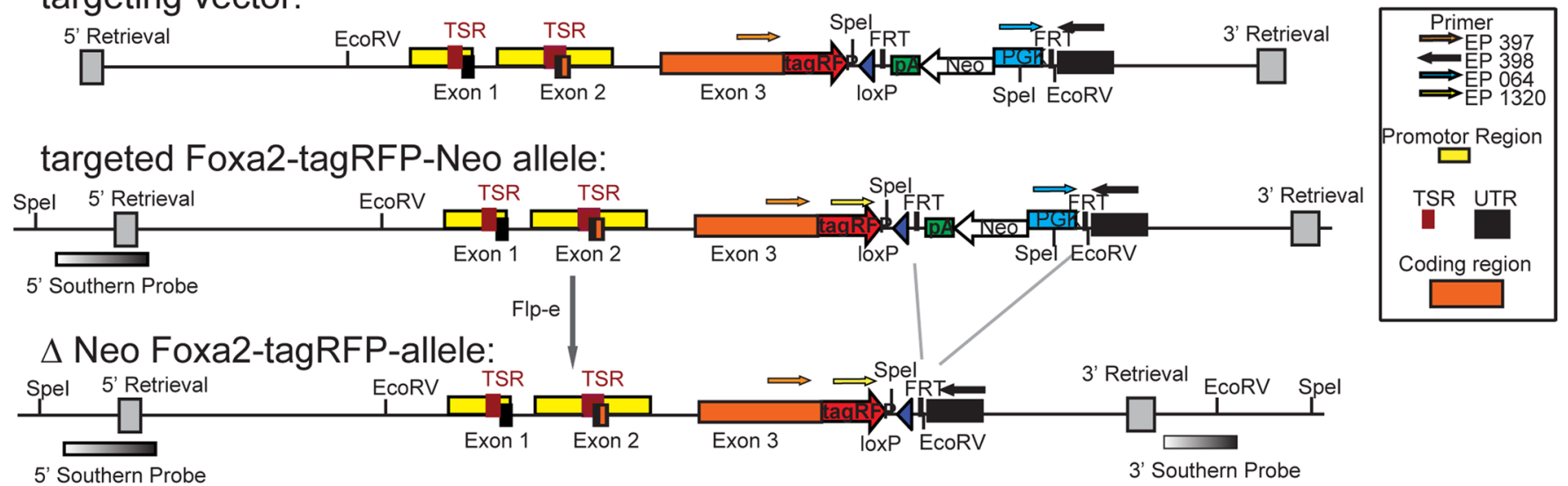

b

Endoderm

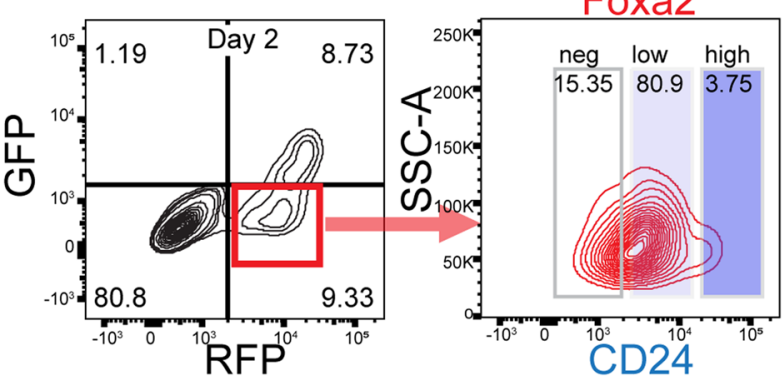

Foxa2 $^{+}$

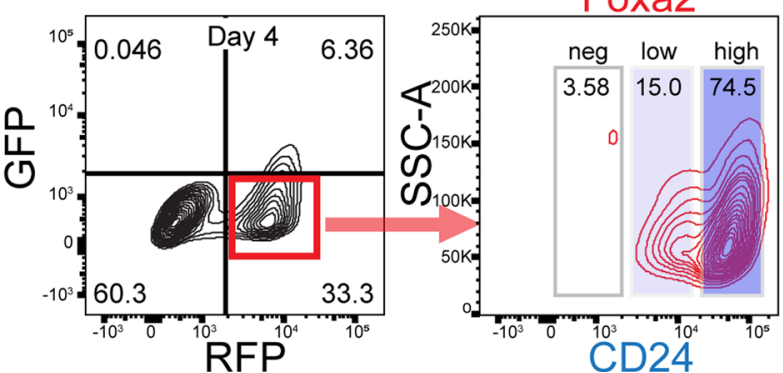

C

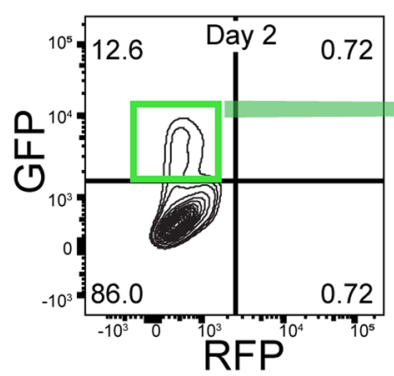

Mesoderm
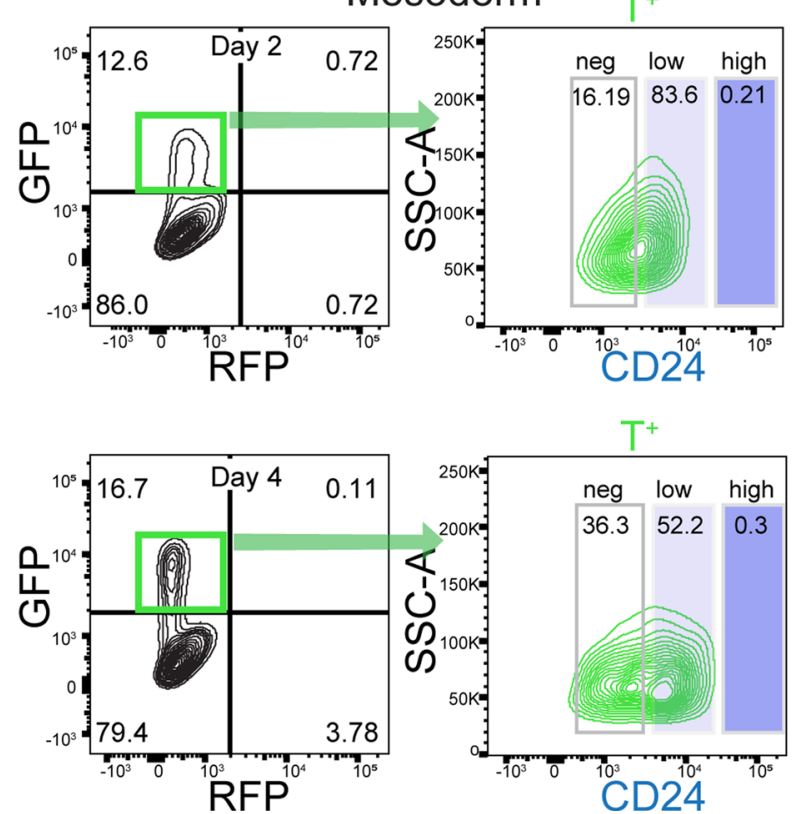

Extended Data Fig. 3 | Characterization of endoderm and mesoderm in vitro. a, Targeting strategy to generate TGFP/+; Foxa2tagRFP/+ $m E S C$ line. An available T-GFP knock-in mESCs line ${ }^{62}$ was targeted with the described Foxa2 ${ }^{\text {tagRFP }}$ construct. Transcriptional start region (TSR), untranslated region (UTR). b, Gating strategy of differentiated T $\mathrm{T}^{\mathrm{GFP} /+}$; Foxa2 $2^{\text {tagRFP/+ }}$ mESC line towards Foxa2+ (RFP+) endodermal cells and (c) $\mathrm{T}^{+}(\mathrm{GFP}+)$ mesodermal cells. Differentiated cells were stained for CD24 and sorted according to their CD24 expression levels Foxa2 ${ }^{+} / C D 24^{\text {low }}$, Foxa2 ${ }^{+} / C_{C D} 24^{\text {high }}, T^{+} / C D 24^{\text {low }}$ and $T^{+} / C D 24^{\text {neg }}$ at day 2 and 4 of differentiation for global mRNA expression profile analysis. 


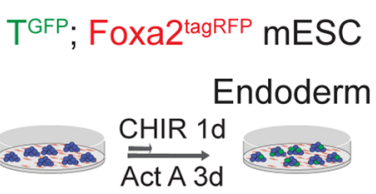

FVF mESC Endoderm

e

Foxa2 ${ }^{\text {Venus/+ }}$ mESC

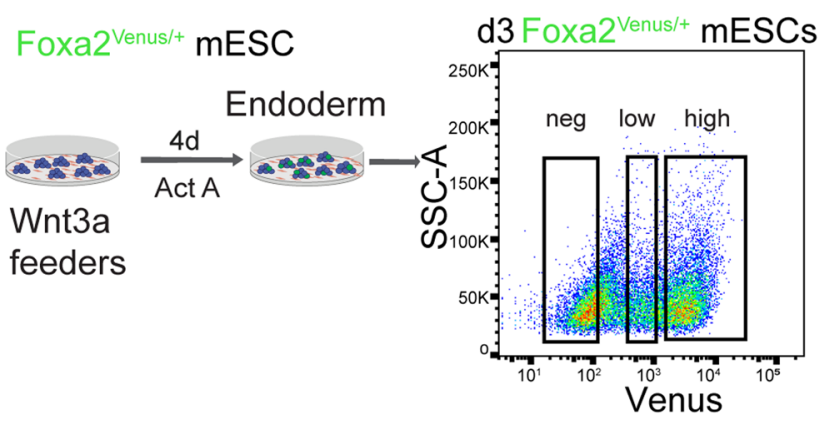

b

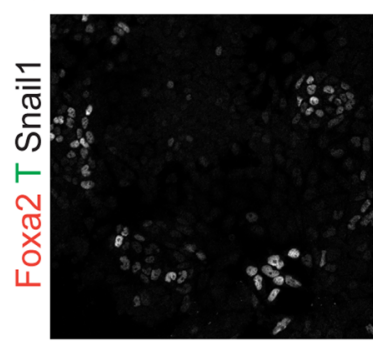

d

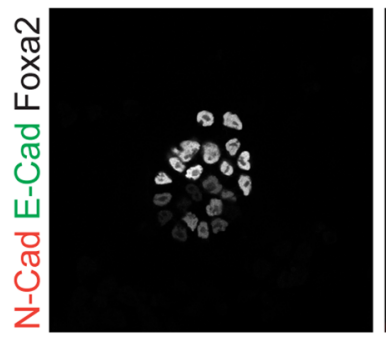

f
TGFP; Foxa2 ${ }^{\text {tagRFP }} \mathrm{mESC}$ day 3
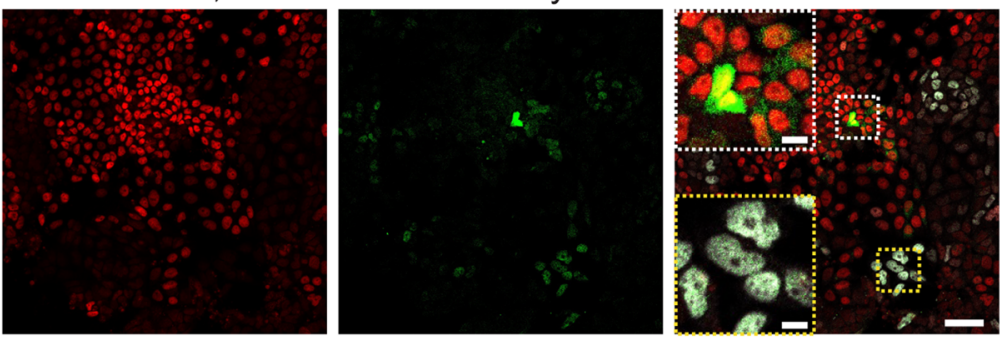

FVF mESC day 3
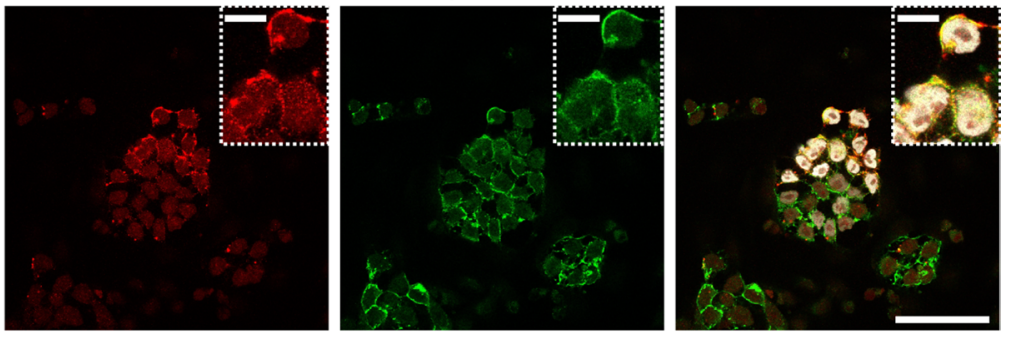

h
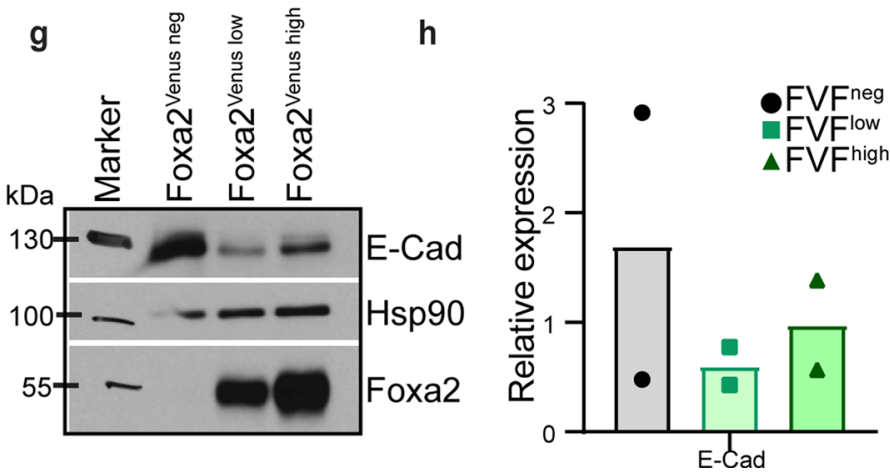

Extended Data Fig. 4 | Endoderm does not show hallmarks of EMT in vitro. a, Endoderm differentiation scheme of TGF/+; Foxa2 $2^{\operatorname{tagRFP} /+} \mathrm{mESC}$ line. b, Confocal images of differentiated TGFP/+; Foxa2 ${ }^{\text {tagRFP/+}} \mathrm{mESC}$ line (representative of 3 independent differentiations) at day 3 stained for GFP (T), Foxa2 and Snail1. Note, the presence of $\mathrm{T}^{+}$, Snail1+ MES population (yellow box), Foxa $2^{\text {high }} / \mathrm{T}^{+} \mathrm{AME}$ progenitors (white box) as well as Foxa $2^{\text {low }} /$ high $D E$ progenitors, recapitulating the observations in vivo. c, Differentiation scheme of FVF mESCs towards endoderm fate. d, Confocal images of differentiated FVF mESCs (representative of 2 independent differentiations) at day 3 stained for Venus (Foxa2, white), E-cadherin and N-cadherin. e, Scheme of endoderm differentiation. f, Representative

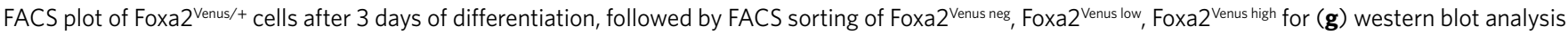
( 2 experiments) and ( $\mathbf{h}$ ) quantification of E-cadherin ( $n=2$ experiments). All samples are derived from biologically independent experiments. Data are presented as mean values. All shown confocal images are single planes of a z-stack unless otherwise stated. Scale bar: $50 \mu \mathrm{m}$, insets $10 \mu \mathrm{m}$. 
wild-type Snail1 allele:
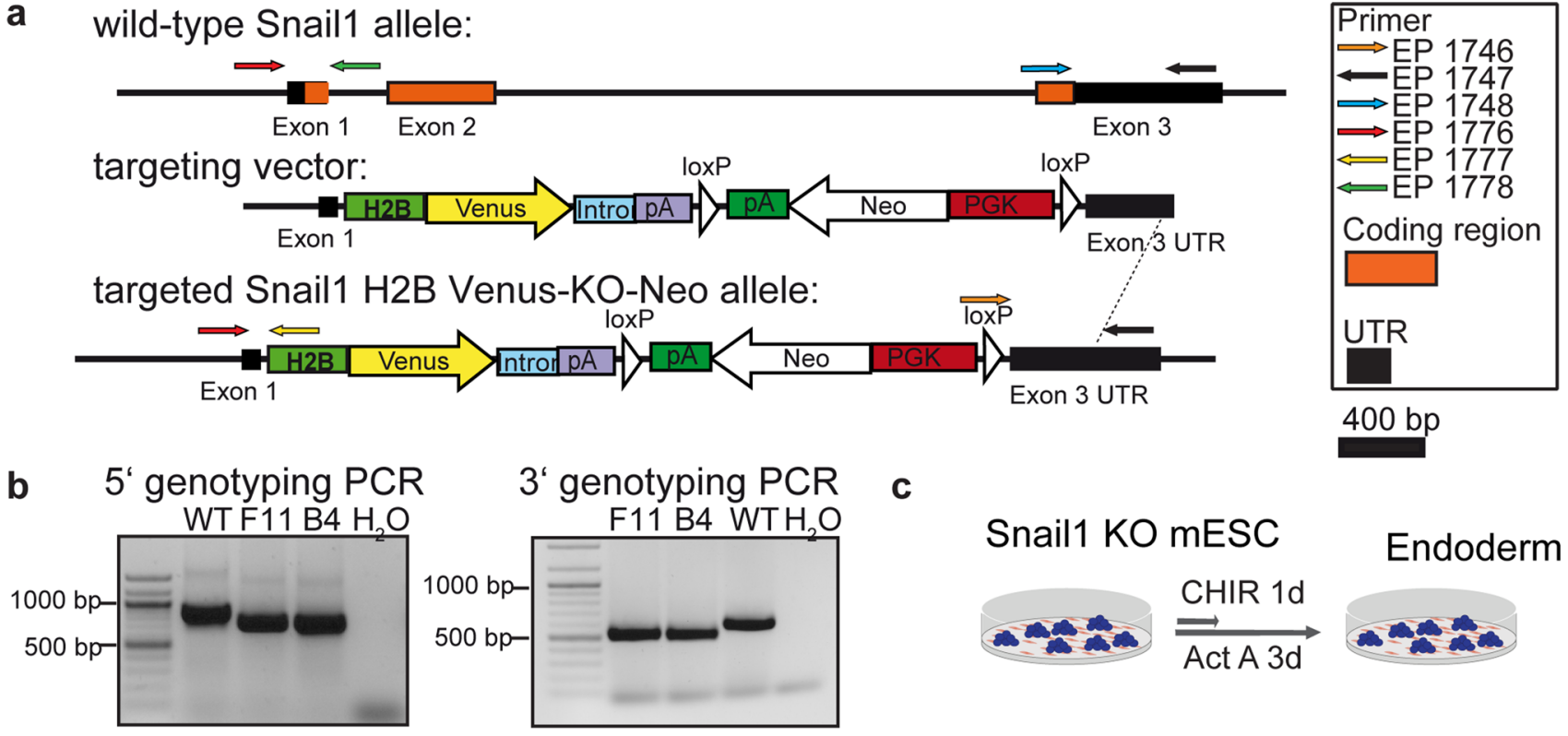

C

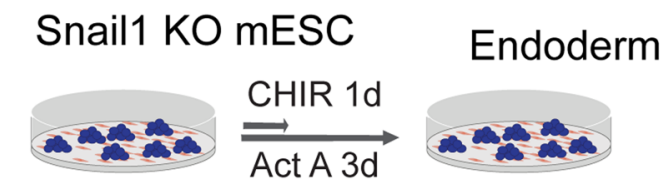

d
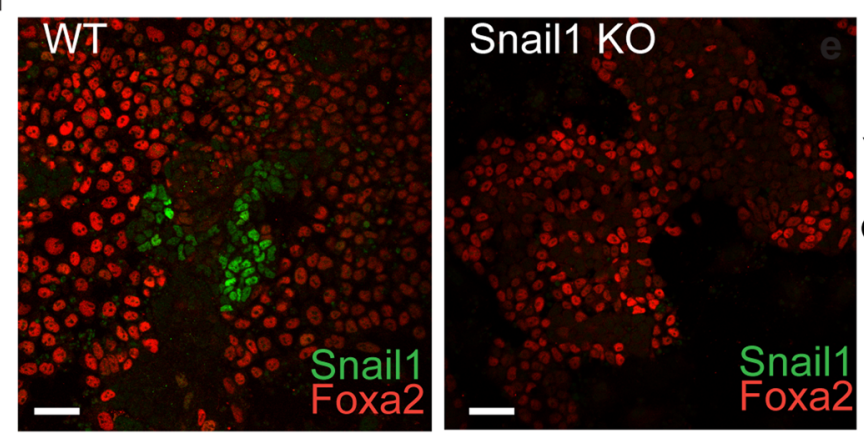

f

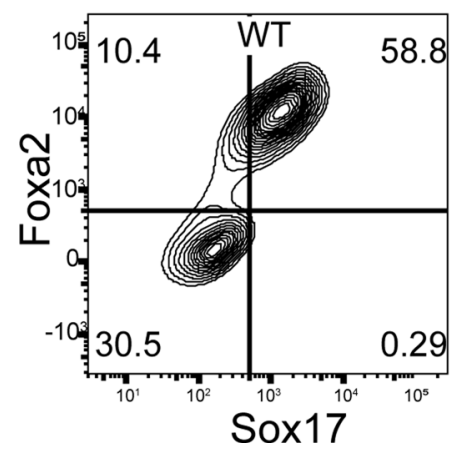

e

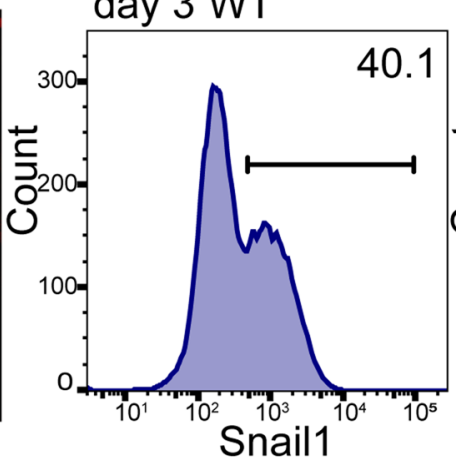

day 3 Snail1 $\mathrm{KO}$

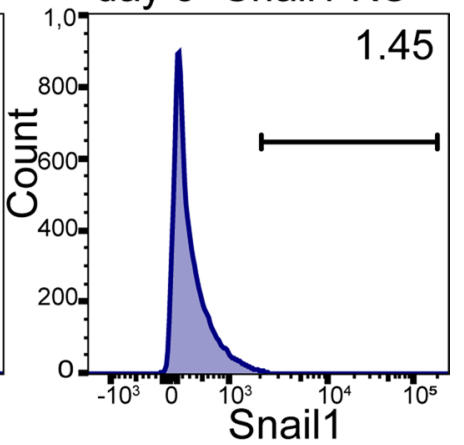

Extended Data Fig. 5 | Generation of Snail1 knockout mESCs. a, Targeting strategy to generate Snail1 KO mESCs. b, 3' end genotyping PCR (WT=601 bp, $\mathrm{KI}=529 \mathrm{bp})$ and $5^{\prime}$ end genotyping PCR (WT $\left.=738 \mathrm{bp}, \mathrm{KI}=657 \mathrm{bp}\right)$ of the used Snail1 KO clones F11 and B4. c, Endoderm differentiation scheme of Snail1 KO mESCs. d, Confocal images of differentiated WT and Snail1 KO mESCs (representative of 2 independent differentiations) at day 3 stained against Snail1 and Foxa2. e, FACS analysis of differentiated WT and Snail1 KO mESCs (2 independent differentiations) at day 3 stained for Snail1 confirming the absence of Snail1 in the Snail1 KO mESCs line. f, FACS analysis of differentiated wildtype and Snail1 KO mESCs for Foxa2 and Sox17 expression ( $n=5$ (wildtype), $\mathrm{n}=8($ Snail1 KO)). All samples are derived from biologically independent experiments. All shown confocal images are single planes of a $z$-stack unless otherwise stated. Scale bar: $50 \mu \mathrm{m}$, insets $10 \mu \mathrm{m}$. 
TGFP $^{\text {Goxa2 }} 2^{\text {tagRFP }} \mathrm{mESC}$

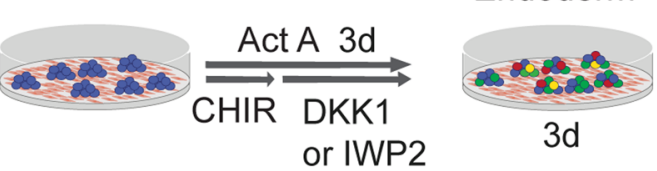

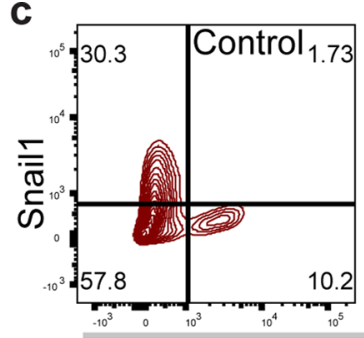

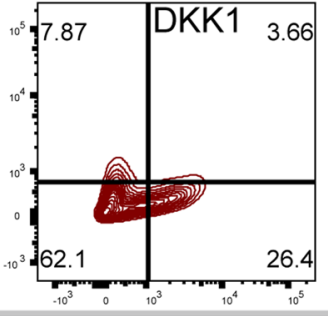

Foxa2 b

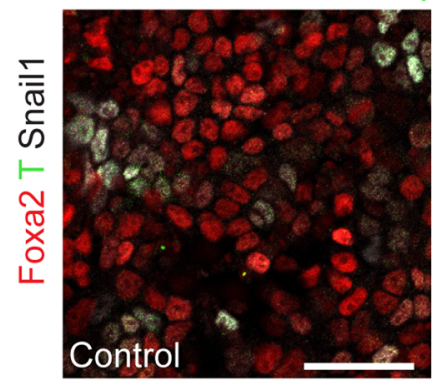

TGFP; Foxa2 $^{\text {tagRFP }}$ mESC day 3
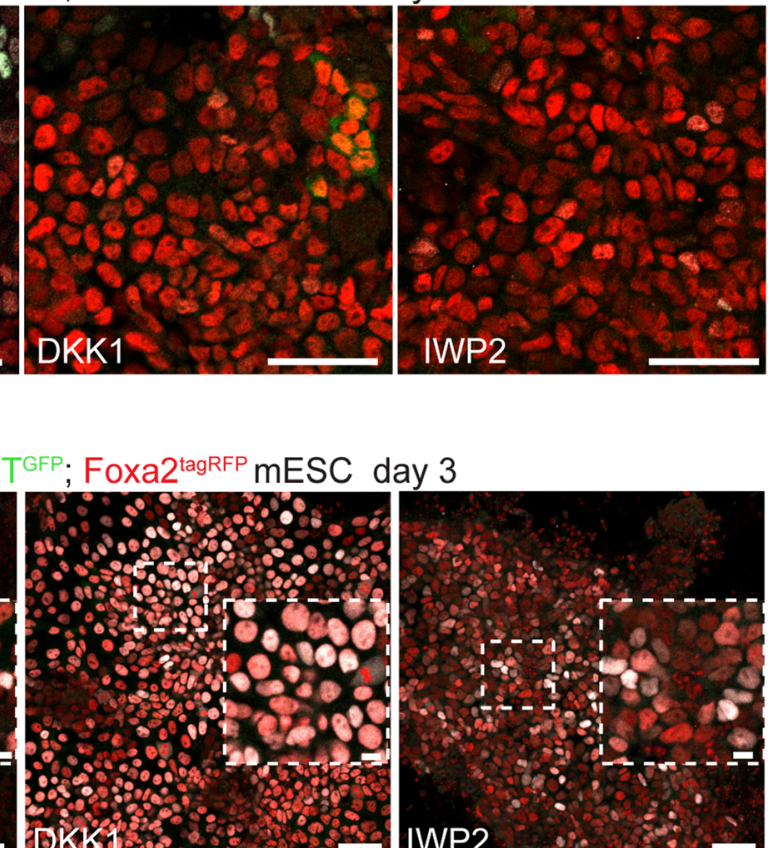

Foxa2 $2^{\text {tagRFP }} \mathrm{mESC}$ day 3

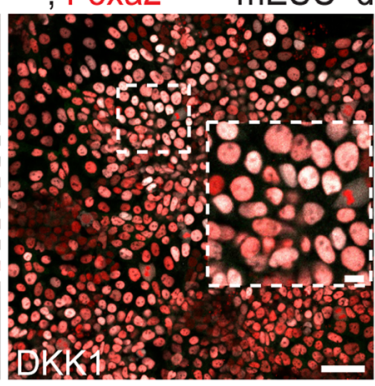

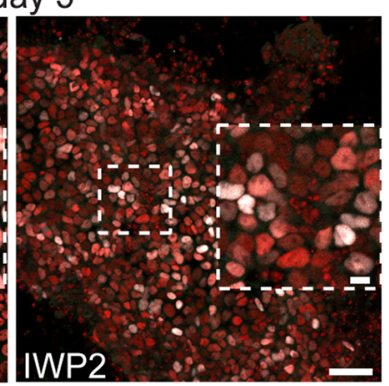

e

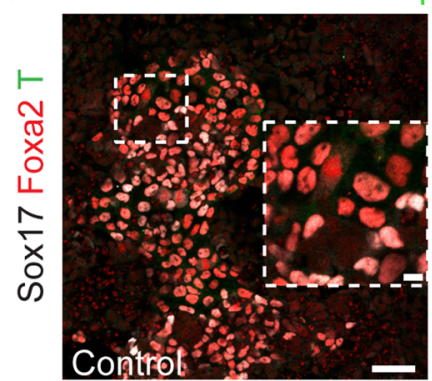

d

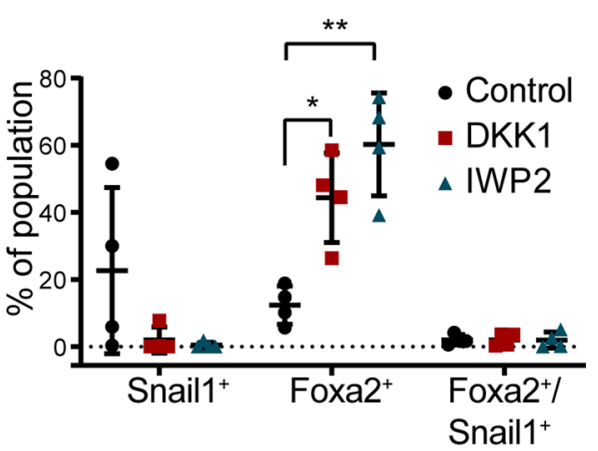

g

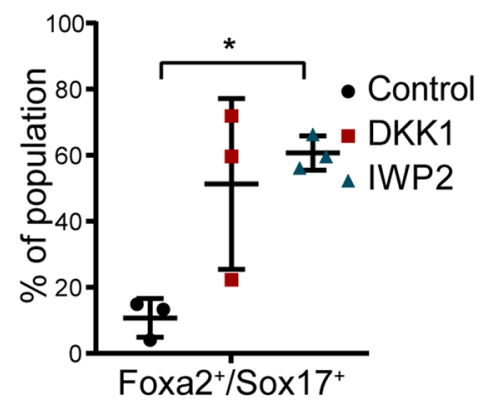

f
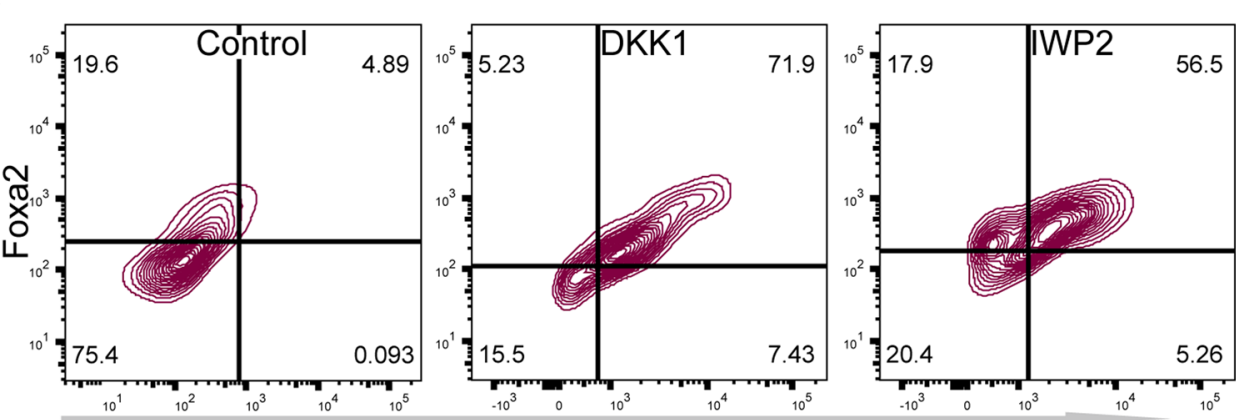

Sox 17

Extended Data Fig. 6 | Inhibition of Wnt signaling promotes endoderm differentiation. a, Scheme of endoderm differentiation under normal conditions (control) and with the supplementation of DKK1 or IWP2. b, Immunohistochemistry (representative of 3 independent differentiations), (c) FACS analysis and (d) quantification of differentiated TGF/+; Foxa $2^{\text {tagRFP/+ }}$ under control, DKK1 and IWP2 conditions stained for GFP (T), Foxa2 and Snail1 (Ordinary one-way ANOVA with Bonferroni's multiple comparison test, $n=4$ independent differentiations). e, Confocal images (representative of 3 independent differentiations), (f) FACS analysis and (g) quantification of $\mathrm{T}^{\mathrm{GFP} /+}$; Foxa2 $2^{\text {tagRFP/+ }} \mathrm{mESC}$ differentiated for 3 days under control, DKK1 or IWP2 conditions and stained for GFP (T), Foxa2 and Sox17 (Ordinary one-way ANOVA with Bonferroni's multiple comparison test, $\mathrm{n}=3$ independent differentiations). All samples are derived from biologically independent experiments. Data are presented as mean values $\pm S E M$. $\mathbf{d},{ }^{\star} P<0.0146,{ }^{\star *} P<0.0011,(\mathbf{g}){ }^{\star} P<0.0233$. Statistically non-significant results are not indicated in the figure. All shown confocal images are single planes of a z-stack unless otherwise stated. Scale bar: $50 \mu \mathrm{m}$, insets $10 \mu \mathrm{m}$. 


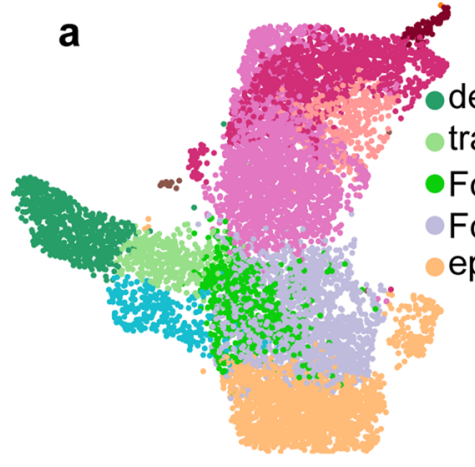

C

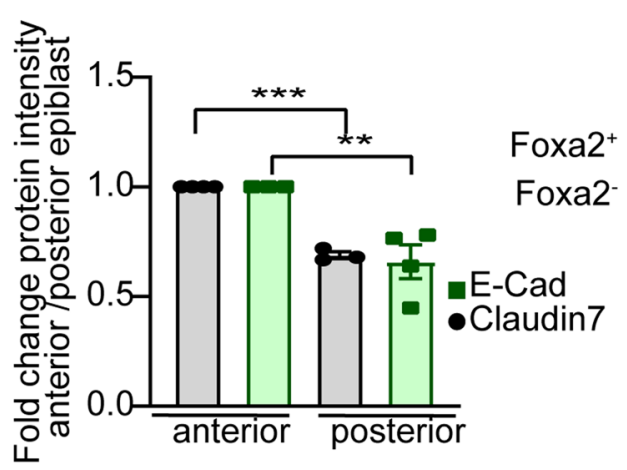

d transitory progenitors (TP)

Foxa2 ${ }^{+}$posterior epiblast (Foxa2 $\left.{ }^{+} \mathrm{pEpi}\right)$

-Foxa2- posterior epiblast (Foxa2- pEpi) epiblast (Epi)
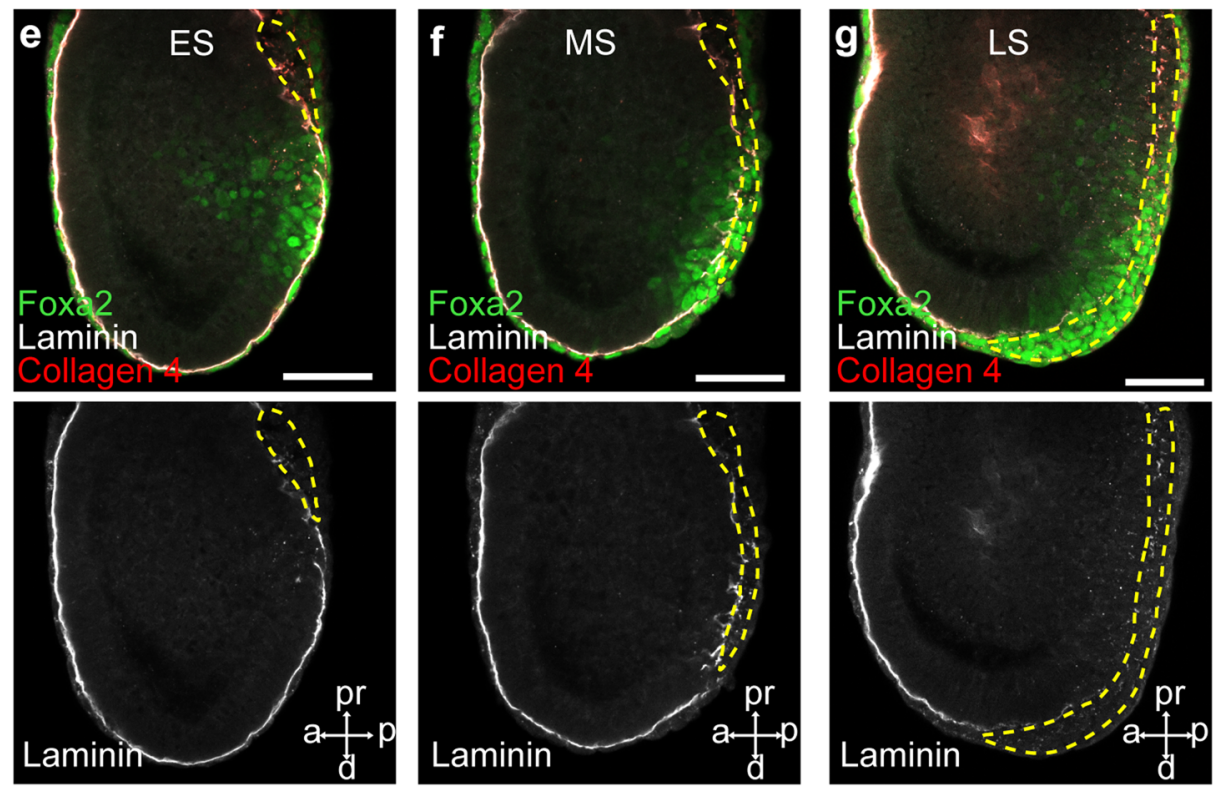

b

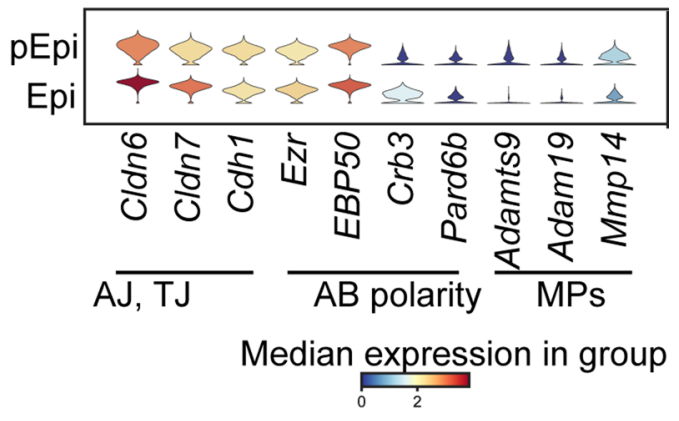

Extended Data Fig. 7 | Epithelial cell plasticity drives endoderm formation. a, UMAP plot colored by cell population. b, Stacked violin plots showing the gene expression distribution (columns) of AJ, TJ, AB polarity and metalloproteinases (MPs) genes in pEpi $(n=2215)$ versus Epi $(n=1929)$. Color corresponds to normalized median gene expression for each group. c, Quantification of E-cadherin (one-way ANOVA, $n=4$ embryos) and Claudin7 (one-way ANOVA, $n=3$ embryos) expression intensity at the anterior and posterior epiblast of MS stage embryos. $\mathbf{d}$, Stacked violin plots showing the expression of TFs, AJ, TJ, AB polarity and metalloproteinases (MPs) genes in Foxa2 ${ }^{+}$pEpi $(n=616)$ versus Foxa2 $p E p i(n=1599)$. e, Immunostainings for Foxa2, Laminin and Collagen 4 of ES, (f) MS and (g) LS stage embryos (representative of 7 embryos used for (e-g). Yellow dashed line indicates PS. h, MS stage embryo (3 embryos) stained for Foxa2, Scribble and E-cadherin. All samples are derived from biologically independent experiments. Data are presented as mean values \pm SEM.; ${ }^{* \star} P<0.0017$, ${ }^{\star \star \star} P<0.0054$. Statistically non-significant results are not indicated in the figure. All shown confocal images are single planes of a z-stack unless otherwise stated. Scale bar: $50 \mu \mathrm{m}$, insets $10 \mu \mathrm{m}$. 


\section{natureresearch}

Corresponding author(s): Heiko Lickert

Last updated by author(s): Apr 19, 2021

\section{Reporting Summary}

Nature Research wishes to improve the reproducibility of the work that we publish. This form provides structure for consistency and transparency in reporting. For further information on Nature Research policies, see Authors \& Referees and the Editorial Policy Checklist.

\section{Statistics}

For all statistical analyses, confirm that the following items are present in the figure legend, table legend, main text, or Methods section.

n/a Confirmed

$\bigotimes$ The exact sample size $(n)$ for each experimental group/condition, given as a discrete number and unit of measurement

$\square \bigotimes$ A statement on whether measurements were taken from distinct samples or whether the same sample was measured repeatedly

The statistical test(s) used AND whether they are one- or two-sided

$\square$ Only common tests should be described solely by name; describe more complex techniques in the Methods section.

Х $\square$ A description of all covariates tested

Х $\square$ A description of any assumptions or corrections, such as tests of normality and adjustment for multiple comparisons

$\triangle$ A full description of the statistical parameters including central tendency (e.g. means) or other basic estimates (e.g. regression coefficient)

AND variation (e.g. standard deviation) or associated estimates of uncertainty (e.g. confidence intervals)

For null hypothesis testing, the test statistic (e.g. $F, t, r$ ) with confidence intervals, effect sizes, degrees of freedom and $P$ value noted

Give P values as exact values whenever suitable.

Х $\square$ For Bayesian analysis, information on the choice of priors and Markov chain Monte Carlo settings

Х $\square$ For hierarchical and complex designs, identification of the appropriate level for tests and full reporting of outcomes

\ $\square$ Estimates of effect sizes (e.g. Cohen's $d$, Pearson's $r$ ), indicating how they were calculated

Our web collection on statistics for biologists contains articles on many of the points above.

\section{Software and code}

Policy information about availability of computer code

Data collection Gene expression was assessed in differentiated and undifferentiated cells by Affymetrix Mouse Gene ST 1.0 arrays (Applied Biosystems) For flow cytometry BD FACS Aria III was used. Images were acquired with Leica SP5 confocal microscope and Zeiss LSM 880 Airy Scan confocal microscope. Western blots were developed with ChemStudio SA2 Imager from Analytik Jena AG.

Data analysis

$$
\begin{aligned}
& \text { Microarray data were analysed using Expression Suite Software v.1.2 Affymetrix (Applied Biosystems) and R (3.6.3)/Bioconductor (3.9) } \\
& \text { using packages oligo (1.480) and limma (3.40.6) for differential expression analysis. FACS analysis was done via FlowJo }{ }^{\text {TM }} \mathrm{v} 10.2 \text {. Images } \\
& \text { were acquired with Leica SP5 confocal microscope and Zeiss LSM } 880 \text { Airy Scan confocal microscope. Images taken by Leica confocal } \\
& \text { were analysed using Leica LAS AF Lite 4.0. Images taken by Zeiss confocal microscope were analysed using Zeiss Zen } 2.3 \text { lite Blue } \\
& \text { software. For quantifications of confocal images and Western Blot ImageJ v1.53c software was used. All statistics were performed using } \\
& \text { GraphPad Prism software } 8 \text { (GraphPad Software Inc., La Jolla, CA). ChIP-seq data were analysed using Trimmomatic (0.39), Bowtie2 } \\
& \text { (2.3.5.1) and GEM (3.4). ChIP-seq and ATAC-seq data were visulised using bigWigMerge (2) and Gviz (1.28.3). RNA-seq data were } \\
& \text { analysed using R (3.6.3)/Bioconductor (3.9) and the packages tximport (1.12.3), RUVseq (1.18.0), DESeq2 (1.20.0) and apeglm (1.6.0). } \\
& \text { Single-cell RNAseq data were analyzed using scanpy versions 1.4.5.2.dev6+gfa408dc7, 1.5.1 and 1.6, anndata version 0.7.1, scanorama } \\
& \text { version 1.4, gProfiler-official version 1.0.0, scVelo version 0.2.1, CellRank version 1.0.0-rc.0 and Python 3.7 and 3.8. The data analyses of } \\
& \text { scRNA-seq data are available at https://github.com/theislab/gastrulation_analysis. }
\end{aligned}
$$


Policy information about availability of data

All manuscripts must include a data availability statement. This statement should provide the following information, where applicable:

- Accession codes, unique identifiers, or web links for publicly available datasets

- A list of figures that have associated raw data

- A description of any restrictions on data availability

All the data generated or analysed during the current study are included in this published article and its supplementary files. The datasets generated during and/or analysed during the current study are available from the corresponding author on reasonable request. All microarray data is available at GEO under the accession number GSE148226 (currently private and only accessible using accession token: uzmbyasspxmttwt). All single cell RNA sequencing data is available under GSE162534 (currently private and only accessible using accession token: ehivmicwztmjncl). In addition, the following public datasets were analyzed that are available in the GEO repository under the accessions GSE116257 (sample GSM3223321), GSE116258 (samples GSM3223325, GSM3223326) and GSE116260 (samples GSM3223342 to GSM3223345, GSM3597790, GSM3597791)"

\section{Field-specific reporting}

Please select the one below that is the best fit for your research. If you are not sure, read the appropriate sections before making your selection. \ Life sciences Behavioural \& social sciences Ecological, evolutionary \& environmental sciences

For a reference copy of the document with all sections, see nature.com/documents/nr-reporting-summary-flat.pdf

\section{Life sciences study design}

All studies must disclose on these points even when the disclosure is negative.

Sample size No statistical test or power analysis were performed to predetermine sample size. We defined sample sizes based on past experience and based on relevant literature (Mahaddalkar PU, Scheibner K et al., Generation of pancreatic beta cells from CD177+ anterior definitive endoderm, 2020; Böttcher, A., Büttner, M., Tritschler, S. et al. Non-canonical Wnt/PCP signalling regulates intestinal stem cell lineage priming towards enteroendocrine and Paneth cell fates. Nat Cell Biol 23, 23-31 (2021)). In general, all experiments were done, if possible, with at least 3 independent biological samples and sample sizes are provided in figure legends. Less than 3 independent experiments were used for the FVF embryo sorting and WB analysis due to the high quantity of embryos required for this experiment. However, a total number of 158 embryos in two independent experiments was used and considered as sufficient.

Data exclusions Data was excluded when immunohistochemical stainings were insufficient, $m E S C$ differentiations failed $(<2 \%$ Foxa $2+$ cells $)$, embryos were ruptured or at a wrong stage. For scRNA seq analysis, FVF_neg_3 was excluded from analysis due to low sequencing depth.

Replication Every experiment was repeated a minimum of 3 times, if possible. All replications were successful.

Randomization There was randomisation done in collection of samples if possible. For microscopy, embryos that were damaged or embedded in a wrong orientation were excluded from anaylysis. However the whole embryos (excluding extra-embryonic) were imaged and not restricted to a specific region. For quantifications embryos were chosen randomly. For mESC differentiation random positions were chosen to take pictures. For mESC differentiations one dish was used per differentiation and all cells differentiated were anaylsed. For FACS analysis a minimum of 10,000 events were randomly analysed. For sc RNA sequencing of FVF embryos a total of 103 early- to late streak stage embryos were randomly chosen and sorted by FACS.

Blinding

The same investigators performed and analysed the experiments, thus no blinding was performed in our studies.

\section{Behavioural \& social sciences study design}

All studies must disclose on these points even when the disclosure is negative.

Study description

Briefly describe the study type including whether data are quantitative, qualitative, or mixed-methods (e.g. qualitative cross-sectional, quantitative experimental, mixed-methods case study).

Research sample

State the research sample (e.g. Harvard university undergraduates, villagers in rural India) and provide relevant demographic information (e.g. age, sex) and indicate whether the sample is representative. Provide a rationale for the study sample chosen. For studies involving existing datasets, please describe the dataset and source.

Sampling strategy

Describe the sampling procedure (e.g. random, snowball, stratified, convenience). Describe the statistical methods that were used to predetermine sample size $O R$ if no sample-size calculation was performed, describe how sample sizes were chosen and provide a rationale for why these sample sizes are sufficient. For qualitative data, please indicate whether data saturation was considered, and what criteria were used to decide that no further sampling was needed. 
Data collection

computer, eye tracker, video or audio equipment) whether anyone was present besides the participant(s) and the researcher, and whether the researcher was blind to experimental condition and/or the study hypothesis during data collection.

Timing

Indicate the start and stop dates of data collection. If there is a gap between collection periods, state the dates for each sample cohort.

Data exclusions If no data were excluded from the analyses, state so OR if data were excluded, provide the exact number of exclusions and the rationale behind them, indicating whether exclusion criteria were pre-established.

Non-participation State how many participants dropped out/declined participation and the reason(s) given OR provide response rate OR state that no participants dropped out/declined participation.

Randomization If participants were not allocated into experimental groups, state so OR describe how participants were allocated to groups, and if allocation was not random, describe how covariates were controlled.

\section{Ecological, evolutionary \& environmental sciences study design}

All studies must disclose on these points even when the disclosure is negative.

Study description

Research sample

Sampling strategy

Data collection

Timing and spatial scale

Reproducibility

Randomization

Blinding

Did the study involve field work?

Briefly describe the study. For quantitative data include treatment factors and interactions, design structure (e.g. factorial, nested, hierarchical), nature and number of experimental units and replicates.

Describe the research sample (e.g. a group of tagged Passer domesticus, all Stenocereus thurberi within Organ Pipe Cactus National Monument), and provide a rationale for the sample choice. When relevant, describe the organism taxa, source, sex, age range and any manipulations. State what population the sample is meant to represent when applicable. For studies involving existing datasets, describe the data and its source.

Note the sampling procedure. Describe the statistical methods that were used to predetermine sample size OR if no sample-size calculation was performed, describe how sample sizes were chosen and provide a rationale for why these sample sizes are sufficient.

Describe the data collection procedure, including who recorded the data and how.

Indicate the start and stop dates of data collection, noting the frequency and periodicity of sampling and providing a rationale for these choices. If there is a gap between collection periods, state the dates for each sample cohort. Specify the spatial scale from which the data are taken

If no data were excluded from the analyses, state so OR if data were excluded, describe the exclusions and the rationale behind them, indicating whether exclusion criteria were pre-established.

Describe the measures taken to verify the reproducibility of experimental findings. For each experiment, note whether any attempts to repeat the experiment failed OR state that all attempts to repeat the experiment were successful.

Describe how samples/organisms/participants were allocated into groups. If allocation was not random, describe how covariates were controlled. If this is not relevant to your study, explain why.

Describe the extent of blinding used during data acquisition and analysis. If blinding was not possible, describe why OR explain why blinding was not relevant to your study.

Field work, collection and transport

Field conditions

Describe the study conditions for field work, providing relevant parameters (e.g. temperature, rainfall).

Location

State the location of the sampling or experiment, providing relevant parameters (e.g. latitude and longitude, elevation, water depth).

Access and import/export

Describe the efforts you have made to access habitats and to collect and import/export your samples in a responsible manner and in compliance with local, national and international laws, noting any permits that were obtained (give the name of the issuing authority, the date of issue, and any identifying information).

Disturbance

\section{Reporting for specific materials, systems and methods}

We require information from authors about some types of materials, experimental systems and methods used in many studies. Here, indicate whether each material, system or method listed is relevant to your study. If you are not sure if a list item applies to your research, read the appropriate section before selecting a response. 


\begin{tabular}{l|l}
\hline n/a & Involved in the study \\
\hline & $\bigotimes$ Antibodies \\
$\square$ Eukaryotic cell lines \\
$\square$ Palaeontology \\
$\square$ Animals and other organisms \\
$\square$ & $\square$ Human research participants \\
$\square$ & $\square$ Clinical data
\end{tabular}

\begin{tabular}{l|l}
\hline n/a & Involved in the study \\
$\square$ & $\square$ ChIP-seq \\
$\square$ & $\square$ Flow cytometry \\
$\square$ & $\square$ MRI-based neuroimaging
\end{tabular}

\section{Antibodies}

Antibodies used
Mouse CD24-Pacific Blue Biolegend BLD-101819, 1:100

Isotype Control-Pacific Blue, Rat IgG2b, Biolegend, BLD-400627, 1:100

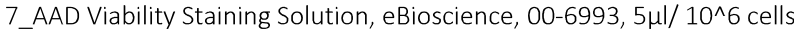

Goat SOX17, Acris/Novus, GT15094 1:1000

Rabbit Foxa2, Cell signalling, 8186, 1:1000

Goat Foxa2, Santa Cruz, sc-6554, 1:1000

Mouse E-cadherin, BD, 610181, 1:1000

Rabbit E-cadherin, Cell Signaling, 3195, 1:1000

Mouse N-cadherin, BD, 610920, 1:1000

Chicken GFP, Aves Labs, GFP-1020, 1:1000

Rabbit RFP, Rockland, 600-401-379, 1:1000

Goat Brachyury, Santa Cruz, sc17743, 1:1000

Rabbit Snail1, Cell signaling, C15D3, 1:500

Rabbit Lef1, Abcam, ab137872, 1:500

Rabbit Hsp90, Cell signaling, 4874S, 1:1000

Rat E-Cadherin, TaKaRa, M108, 1:500

Rat Cer1, R\&D, MAB1986, 1:500

Rabbit Claudin7, LIFE Technologies, 34-9100, 1:500

Mouse Foxa2, GeneTex, GTX84485, 1:500

Rat Laminin, Millipore, MAB1914, 1:500-1:1000

Rat Laminin, Abcam, ab44941, 1:500-1:1000

Rabbit Collagen 4, Abcam, ab6586, 1:1000

Rabbit EBP50, ab3452, 1:300

Rabbit Ezrin/Radixin/Moesin, Cell signaling, 3141, 1:300

Rabbit Scribble, Santa Cruz, sc-28737, 1:300

donkey anti-goat IgG 555, Invitrogen, A21432, 1:800

donkey anti-rat IgG 647, Dianova, 712-605-150, 1:800

Goat Anti-Rabbit IgG (H+L), HRP Dianova/Jackson, 111-035-144, 1:5000

donkey anti-mouse IgG 488, Invitrogen, A21202, 1:800

donkey anti-rabbit IgG 555, Invitrogen, A31572, 1:800

donkey anti-goat IgG 488, Invitrogen, A11055, 1:800

donkey anti-rabbit IgG 488, Invitrogen, A21206, 1:800

donkey anti-chicken IgY, Dianova, 703-225-155,1:800

donkey anti-mouse IgG 555, Invitrogen, A31570, 1:800

All primary antibodies were validated by the manufactures. Further, their expression was tested on undifferentiated cells and/or mouse embryos. Various antibody dilutions were tested including the manufacturer's recommended dilution. The secondary antibody only controls/isotype controls were used to evaluate and confirm specificity of the antibodies to the respective epitopes. All of the antibodies used in this study have been used and reported in prior studies.

primary antibodies:

Mouse CD24-Pacific Blue Biolegend BLD-101819 - https://www.biolegend.com/en-us/products/pacific-blue-anti-mouse-cd24antibody-3584

Isotype Control-Pacific Blue, Rat IgG2b, Biolegend, BLD-400627 - https://www.biolegend.com/en-us/products/pacific-blue-ratigg2b-kappa-isotype-ctrl-3159?GroupID=GROUP29

7_AAD Viability Staining Solution, eBioscience, 00-6993 - https://www.fishersci.ca/shop/products/7-aad-viability-stainingsolution/501128859

Goat SOX17, Acris/Novus, GT15094 - https://www.novusbio.com/products/sox17-antibody_af1924

Rabbit Foxa2, Cell signalling, 8186 - https://www.cellsignal.com/products/primary-antibodies/foxa2-hnf3b-d56d6-xp-rabbitmab/8186

Goat Foxa2, Santa Cruz, sc-6554 - https://www.scbt.com/p/hnf-3beta-antibody-m-20

Mouse E-cadherin, BD, 610181 - https://www.bdbiosciences.com/us/applications/research/stem-cell-research/cancer-research/ human/purified-mouse-anti-e-cadherin-36e-cadherin/p/610181 
Rabbit E-cadherin, Cell Signaling, 3195 - https://www.cellsignal.com/products/primary-antibodies/e-cadherin-24e10-rabbitmab/3195

Mouse N-cadherin, BD, 610920 - https://www.bdbiosciences.com/us/applications/research/stem-cell-research/cancer-research/ human/purified-mouse-anti-n-cadherin-32n-cadherin/p/610920

Chicken GFP, Aves Labs, GFP-1020 - https://www.aveslabs.com/products/anti-green-fluorescent-protein-antibody-gfp Rabbit RFP, Rockland, 600-401-379 - https://rockland-inc.com/store/Antibodies-to-GFP-and-Antibodies-to-RFP-600-401-379O4L 24299.aspx

Goat Brachyury, Santa Cruz, sc17743 - https://www.scbt.com/p/brachyury-antibody-n-19

Rabbit Snail1, Cell signaling, C15D3 - https://www.cellsignal.com/products/primary-antibodies/snail-c15d3-rabbit-mab/3879

Rabbit Lef1, Abcam, ab137872 - https://www.abcam.com/lef1-antibody-epr2029y-ab137872.html

Rabbit Hsp90, Cell signaling, 4874S - https://www.cellsignal.com/products/primary-antibodies/hsp90-antibody/4874

Rat E-Cadherin, TaKaRa, M108 - https://www.takarabio.com/products/antibodies-and-elisa/primary-antibodies-and-elisas-by-

research-area/cell-adhesion-and-ecm/cadherin

Rat Cer1, R\&D, MAB1986 - https://www.rndsystems.com/products/mouse-cerberus-1-antibody-225807 mab1986

Rabbit Claudin7, LIFE Technologies, 34-9100 - https://www.thermofisher.com/antibody/product/Claudin-7-Antibody-

Polyclonal/34-9100

Mouse Foxa2, GeneTex, GTX84485 - https://www.genetex.com/Product/Detail/FOXA2-antibody-3C10/GTX84485

Rat Laminin, Millipore, MAB1914 - https://www.merckmillipore.com/DE/de/product/Anti-Laminin-gamma-1-Antibody-clone-

A5,MM_NF-MAB1914P?ReferrerURL=https\%3A\%2F\%2Fwww.google.com\%2F\&bd=1

Rat Laminin, Abcam, ab44941 - https://www.abcam.com/laminin-beta-1-antibody-lt3-ab44941.html

Rabbit Collagen 4, Abcam, ab6586 - https://www.abcam.com/collagen-iv-antibody-ab6586.html

Rabbit EBP50, ab3452 - https://www.abcam.com/ebp50nherf-1-antibody-ab3452.html

Rabbit Ezrin/Radixin/Moesin, Cell signaling, 3141 - https://www.cellsignal.com/products/primary-antibodies/phospho-ezrin-

thr567-radixin-thr564-moesin-thr558-antibody/3141

Rabbit Scribble, Santa Cruz, sc-28737 - https://www.scbt.com/p/scrib-antibody-h-300

secondary antibodies:

donkey anti-goat IgG 555, Invitrogen, A21432 - https://www.thermofisher.com/antibody/product/Donkey-anti-Goat-IgG-H-LCross-Adsorbed-Secondary-Antibody-Polyclonal/A-21432

donkey anti-rat IgG 647, Dianova, 712-605-150 - https://www.dianova.com/en/shop/712-605-150-donkey-igg-anti-rat-igg-hlalexa-fluor-647-minx-bockgogphshohurbsh/

Goat Anti-Rabbit IgG (H+L), HRP Dianova/Jackson, 111-035-144 - https://www.dianova.com/shop/111-035-144-ziege-igg-antikaninchen-igg-hl-hrpo-minx-humsrt/

donkey anti-mouse IgG 488, Invitrogen, A21202 - https://www.thermofisher.com/order/genome-database/generatePdf? productName=Mouse\%20lgG\%20(H+L)\&assayType=PRANT\&productld=A-21202\&detailed=true

donkey anti-rabbit IgG 555, Invitrogen, A31572 - https://www.thermofisher.com/antibody/product/Donkey-anti-Rabbit-lgG-H-LHighly-Cross-Adsorbed-Secondary-Antibody-Polyclonal/A-31572

donkey anti-goat IgG 488, Invitrogen, A11055 - https://www.thermofisher.com/antibody/product/Donkey-anti-Goat-lgG-H-L-

Cross-Adsorbed-Secondary-Antibody-Polyclonal/A-11055

donkey anti-rabbit IgG 488, Invitrogen, A21206 - https://www.thermofisher.com/antibody/product/Donkey-anti-Rabbit-IgG-H-LHighly-Cross-Adsorbed-Secondary-Antibody-Polyclonal/A-21206

donkey anti-chicken IgY, Dianova, 703-225-155 - https://www.dianova.com/downloads/Jackson/703-225-155.pdf

donkey anti-mouse IgG 555, Invitrogen, A31570 - https://www.thermofisher.com/order/genome-database/generatePdf? productName=Mouse $\% 20 \lg G \% 20(\mathrm{H}+\mathrm{L})$ \&assayType=PRANT\&productld=A-31570\&detailed $=$ true

\section{Eukaryotic cell lines}

Policy information about cell lines

Cell line source(s)

The cell lines used are TGFP mESCs (Fehling et al., 2003), TGFP/+;Foxa2tagRFP/+ mESC were generated in the lab, FVF mESCs (Burtscher et al., 2013), Snail1 KO mESCs were generated in the lab. IDG3.2 mESCs (Hitz et al., 2007), Foxa2 KO mESCs (Cernilogar et al. 2019).

\section{Authentication}

Mycoplasma contamination

Commonly misidentified lines (See ICLAC register)
No authentication was done.

All cell lines were mycoplasma negative

No misidentified cell lines were used.

\section{Palaeontology}

Specimen provenance

Provide provenance information for specimens and describe permits that were obtained for the work (including the name of the issuing authority, the date of issue, and any identifying information).

Specimen deposition 
Dating methods

If new dates are provided, describe how they were obtained (e.g. collection, storage, sample pretreatment and measurement), where they were obtained (i.e. lab name), the calibration program and the protocol for quality assurance OR state that no new dates are provided.

Tick this box to confirm that the raw and calibrated dates are available in the paper or in Supplementary Information.

\section{Animals and other organisms}

Policy information about studies involving animals; ARRIVE guidelines recommended for reporting animal research

Laboratory animals

Wild animals

Field-collected samples

Ethics oversight
Mus musculus: CD-1, mTmG reporter (C57BI/6J; Muzumdar et al., 2007), FVF (C57BI/6];

Burtscher et al., 2013), SCF reporter (C57BI/6J; Burtscher et al., 2012). Age of females: $\geq 6$ weeks, age of males: $\geq 8$ weeks.

No wild animals were used in this study.

No field-collected samples were used in this study.

Animal experiments were performed at the central facilities at HMGU in accordance with the German animal welfare legislation and acknowledged guidelines of the Society of Laboratory Animals (GV-SOLAS) and of the Federation of Laboratory Animal Science Associations (FELASA).

Note that full information on the approval of the study protocol must also be provided in the manuscript.

\section{Human research participants}

Policy information about studies involving human research participants

Population characteristics Describe the covariate-relevant population characteristics of the human research participants (e.g. age, gender, genotypic information, past and current diagnosis and treatment categories). If you filled out the behavioural \& social sciences study design questions and have nothing to add here, write "See above."

Recruitment

Describe how participants were recruited. Outline any potential self-selection bias or other biases that may be present and how these are likely to impact results.

Ethics oversight

Identify the organization(s) that approved the study protocol.

Note that full information on the approval of the study protocol must also be provided in the manuscript.

\section{Clinical data}

Policy information about clinical studies

All manuscripts should comply with the ICMJE guidelines for publication of clinical research and a completed CONSORT checklist must be included with all submissions.

Clinical trial registration

Provide the trial registration number from ClinicalTrials. gov or an equivalent agency.

Study protocol

Note where the full trial protocol can be accessed OR if not available, explain why.

Data collection

Describe the settings and locales of data collection, noting the time periods of recruitment and data collection.

Outcomes

Describe how you pre-defined primary and secondary outcome measures and how you assessed these measures.

\section{ChIP-seq}

\section{Data deposition}

$\square$ Confirm that both raw and final processed data have been deposited in a public database such as GEO.

$\square$ Confirm that you have deposited or provided access to graph files (e.g. BED files) for the called peaks.

Data access links

May remain private before publication.

Files in database submission

Genome browser session (e.g. UCSC)

Methodology

Replicates
For "Initial submission" or "Revised version" documents, provide reviewer access links. For your "Final submission" document, provide a link to the deposited data.

Provide a list of all files available in the database submission.

Provide a link to an anonymized genome browser session for "Initial submission" and "Revised version" documents only, to enable peer review. Write "no longer applicable" for "Final submission" documents. 
Sequencing depth

Antibodies

Peak calling parameters

Data quality

Software
Describe the sequencing depth for each experiment, providing the total number of reads, uniquely mapped reads, length of reads and whether they were paired-or single-end.

Describe the antibodies used for the ChIP-seq experiments; as applicable, provide supplier name, catalog number, clone name, and lot number.

Specify the command line program and parameters used for read mapping and peak calling, including the ChIP, control and index files used.

Describe the methods used to ensure data quality in full detail, including how many peaks are at FDR 5\% and above 5-fold enrichment.

Describe the software used to collect and analyze the ChIP-seq data. For custom code that has been deposited into a community repository, provide accession details.

\section{Flow Cytometry}

Plots

Confirm that:

$\bigotimes$ The axis labels state the marker and fluorochrome used (e.g. CD4-FITC).

$\bigotimes$ The axis scales are clearly visible. Include numbers along axes only for bottom left plot of group (a 'group' is an analysis of identical markers).

$\bigotimes$ All plots are contour plots with outliers or pseudocolor plots.

$\bigotimes$ A numerical value for number of cells or percentage (with statistics) is provided.

\section{Methodology}

Sample preparation

Instrument

Software

Cell population abundance

Gating strategy
Single cell suspensions of undifferentiated and/or S1-S7 differentiated mouse ES cells were fixed, permeabilized and stained with the mentioned primary and secondary antibodies or conjugated antibodies and their isotype controls.

\section{BD FACS Aria III}

FlowJo v10.2

FACS sorting was performed on differentiated T-GFP/Foxa2-RFP mESC. These cells were sorted directly in QIAzol Lysis Reagent for RNA extraction for Affymetrix microarray analysis.

In the preliminary FSC/SSC plot the gates were placed surrounding the main population and to gate for positive/negative populations gatings were put based on the Isotype or secondary antibody control.

$\bigotimes$ Tick this box to confirm that a figure exemplifying the gating strategy is provided in the Supplementary Information.

\section{Magnetic resonance imaging}

\section{Experimental design}

Design type

Design specifications

Behavioral performance measures

Acquisition

Imaging type(s)

Field strength

Sequence \& imaging parameters

Area of acquisition

Diffusion MRI

Used
Indicate task or resting state; event-related or block design.

Specify the number of blocks, trials or experimental units per session and/or subject, and specify the length of each tria or block (if trials are blocked) and interval between trials.

State number and/or type of variables recorded (e.g. correct button press, response time) and what statistics were used to establish that the subjects were performing the task as expected (e.g. mean, range, and/or standard deviation across subjects). 


\section{Preprocessing}

Preprocessing software

Normalization

\section{Normalization template}

Noise and artifact removal

Volume censoring
Provide detail on software version and revision number and on specific parameters (model/functions, brain extraction, segmentation, smoothing kernel size, etc.).

If data were normalized/standardized, describe the approach(es): specify linear or non-linear and define image types used for transformation OR indicate that data were not normalized and explain rationale for lack of normalization.

Describe the template used for normalization/transformation, specifying subject space or group standardized space (e.g. original Talairach, MNI305, ICBM152) OR indicate that the data were not normalized.

Describe your procedure(s) for artifact and structured noise removal, specifying motion parameters, tissue signals and physiological signals (heart rate, respiration).

Define your software and/or method and criteria for volume censoring, and state the extent of such censoring.

Statistical modeling \& inference

Model type and settings

Specify type (mass univariate, multivariate, RSA, predictive, etc.) and describe essential details of the model at the first and second levels (e.g. fixed, random or mixed effects; drift or auto-correlation).

Effect(s) tested

Define precise effect in terms of the task or stimulus conditions instead of psychological concepts and indicate whether ANOVA or factorial designs were used.

Specify type of analysis: Whole brain

ROI-based

Both

Statistic type for inference

(See Eklund et al. 2016)

Specify voxel-wise or cluster-wise and report all relevant parameters for cluster-wise methods.

Correction

Describe the type of correction and how it is obtained for multiple comparisons (e.g. FWE, FDR, permutation or Monte Carlo).

Models \& analysis

$\mathrm{n} / \mathrm{a}$ Involved in the study

$\square$ Functional and/or effective connectivity

$\square$ Graph analysis

$\square$ Multivariate modeling or predictive analysis

Functional and/or effective connectivity

Report the measures of dependence used and the model details (e.g. Pearson correlation, partial correlation, mutual information).

Graph analysis

Report the dependent variable and connectivity measure, specifying weighted graph or binarized graph subject-or group-level, and the global and/or node summaries used (e.g. clustering coefficient, efficiency, etc.).

Multivariate modeling and predictive analysis

Specify independent variables, features extraction and dimension reduction, model, training and evaluation metrics. 